\title{
Primordial non-Gaussian features from DBI Galileon inflation
}

\author{
Sayantan Choudhury ${ }^{1,2, a}$, Supratik Pal ${ }^{2, b}$ \\ ${ }^{1}$ Department of Theoretical Physics, Tata Institute of Fundamental Research, Homi Bhabha Road, Colaba, Mumbai 400005, India \\ 2 Physics and Applied Mathematics Unit, Indian Statistical Institute, 203 B.T. Road, Kolkata 700 108, India
}

Received: 6 January 2015 / Accepted: 10 May 2015 / Published online: 3 June 2015

(C) The Author(s) 2015. This article is published with open access at Springerlink.com

\begin{abstract}
We have studied primordial non-Gaussian features of a model of potential-driven single field DBI Galileon inflation. We have computed the bispectrum from the threepoint correlation function considering all possible cross correlations between the scalar and tensor modes of the proposed setup. Further, we have computed the trispectrum from a fourpoint correlation function considering the contribution from contact interaction, and scalar and graviton exchange diagrams in the in-in picture. Finally we have obtained the nonGaussian consistency conditions from the four-point correlator, which results in partial violation of the SuyamaYamaguchi four-point consistency relation. This further leads to the conclusion that sufficient primordial non-Gaussianities can be obtained from DBI Galileon inflation.
\end{abstract}

\section{Contents}

1 Introduction ................. 1

2 The background model . . . . . . . . . . . . 2

3 Tree level bispectrum analysis . . . . . . . . . . . 3

3.1 Three-scalar correlation . . . . . . . . . . . . 3

3.2 One-scalar two-tensor correlation . . . . . . . . 6

3.3 Two-scalar one-tensor correlation . . . . . . . . 8

3.4 Three-tensor correlation . . . . . . . . . . . . . 9

4 Tree level trispectrum analysis from four-scalar correlation . . . . . . . . . . . . . 10

4.1 Contact interaction . . . . . . . . . 11

4.2 Scalar exchange . . . . . . . . . . . . 13

4.3 Graviton exchange . . . . . . . . . . . . . 14

5 Four-point consistency conditions and violation of Suyama-Yamaguchi relation . . . . . . . . 16

6 Summary and outlook . . . . . . . . . . . . 17

Appendix . . . . . . . . . . . . . . . . . 18

A. Functions appearing in three-scalar correlation . . 18

\footnotetext{
a e-mails: sayantan@theory.tifr.res.in; sayanphysicsisi@gmail.com

b e-mail: supratik@isical.ac.in
}

B. Functions appearing in the one-scalar two-tensor correlation . . . . . . . . . . . . . . 19

C. Functions appearing in two scalar one tensor correlation ................ 21

D. Functions appearing in three-tensor correlation . 24

E. Functions appearing in four-scalar correlator . . . 25

1. Contact interaction . . . . . . . . . 25

2. Scalar exchange . . . . . . . . . . . 26 26

3. Graviton exchange . . . . . . . . . . 27

References . . . . . . . . . . . . . . 28

\section{Introduction}

The physics of the early universe is a very rich area of theoretical physics, for there is a plethora of potential models that solve, at least partially, the well-known problems of the standard cosmological paradigm. Inflationary cosmology is the most successful branch which addressed all of these problems meticulously. This can, however, be explained by several classes of models originating from a proper field theoretic or particle physics framework. But from an observational point view a big issue may crop up in model discrimination and also in the removal of the degeneracy of cosmological parameters obtained from Cosmic Microwave Background (CMB) observations [1-5]. In this context the study of primordial non-Gaussian feature acts as a powerful computational tool to discriminate among inflationary models. In the very recent days the analysis of the bispectrum and the trispectrum derived from the study of primordial features of non-Gaussianity [6-54] from different models of inflation has thus become an intriguing aspect in the context of inflationary model building as well as studies of CMB physics.

Galileon based inflationary models [55-57] and DBI inflationary models $[58,59]$ have both been in vogue for quite some time now. Despite its success, Galileon models generically give rise to unwanted degrees of freedom like ghosts, Laplacian and tachyonic instabilities. Recently, a natural extension to these class of models has been brought forth by 
the present authors [60] in which DBI was clubbed together with Galileon. The framework, called the DBI Galileon framework, consists of a D3 brane in the background of $\mathcal{N}=1, \mathcal{D}=4$ SUGRA derived from the $\mathrm{D} 4$ brane in $\mathcal{N}=2$, $\mathcal{D}=5$ bulk SUGRA background. The interesting feature of this treaty is that this unwanted debris can be successfully thrown away keeping all the good features of the Galileon intact. In the present paper, our prime objective is to investigate some more interesting features of this rich structure of the DBI Galileon [60], which ultimately results in sufficient non-Gaussianity in this framework. Specifically, we explicitly calculate the bispectrum and the trispectrum from threeand four-point correlation functions by exploiting third- and fourth-order actions. The calculations reveal, along with the feature of large non-Gaussianity, some other interesting results like the partial violation of the Suyama-Yamaguchi four-point consistency relation. Subsequently, we demonstrate that, in this framework, it is possible to have a parameter space for both non-Gaussianity and tensor-to-scalar ratio $(r)$ consistent with the combined constraint obtained from the Planck + WMAP9 + high-L + BICEP2 data [2-5].

The plan of the paper is as follows. First we explore primordial non-Gaussian features from the third-order action through the nonlinear parameter $f_{\mathrm{NL}}$ calculated from the bispectrum (in equilateral limit configuration) including all possible scalar-tensor type of cross correlations in the different polarizing modes. Hence from the fourth-order action we derive the expression for the other two nonlinear parameters $g_{\mathrm{NL}}$ and $\tau_{\mathrm{NL}}$ through a trispectrum analysis considering the contribution from contact interaction and scalar and graviton exchange diagrams in the in-in picture. Finally, we explicitly derive the four-point consistency relation from the scalar and graviton exchange diagrams and also find a partial violation of the standard Suyama-Yamaguchi relation [61,62]. We also attempt to give some possible explanations for this violation. We end up with scanning the parameter space for non-Gaussianity and the tensor-to-scalar ratio in the light of Planck + WMAP9 + high-L $+B I C E P 2$ data.

\section{The background model}

For systematic development of the formalism, let us briefly review from our previous paper [60] how one can construct the effective 4D inflationary potential for the DBI Galileon starting from $\mathcal{N}=2, \mathcal{D}=5$ SUGRA along with GaussBonnet correction in the bulk geometry and D4 brane setup leads to an effective $\mathcal{N}=1, \mathcal{D}=4$ SUGRA in the D3 brane. Here the total five dimensional model is described by the following action:

$$
S_{\mathrm{Total}}^{(5)}=S_{\mathrm{EH}}^{(5)}+S_{\mathrm{GB}}^{(5)}+S_{\mathrm{DBI}}^{(5)}+S_{\mathrm{WZ}}^{(5)}+S_{\mathrm{BSUG}}^{(5)}
$$

where

$$
\begin{aligned}
& S_{\mathrm{EH}}^{(5)}=\frac{1}{2 \kappa_{5}^{2}} \int \mathrm{d}^{5} x \sqrt{-g^{(5)}}\left[R_{(5)}-2 \Lambda_{5}\right], \quad S_{\mathrm{GB}}^{(5)} \\
& =\frac{\alpha_{(5)}}{2 \kappa_{5}^{2}} \int \mathrm{d}^{5} x \sqrt{-g^{(5)}}\left[R^{A B C D(5)} R_{A B C D}^{(5)}\right. \\
& \left.-4 R^{A B(5)} R_{A B}^{(5)}+R_{(5)}^{2}\right] \\
& S_{\mathrm{DBI}}^{(5)}=-\frac{T_{4}}{2} \int \mathrm{d}^{5} x \exp (-\Phi) \sqrt{-\left(\gamma^{(5)}+B^{(5)}+2 \pi \alpha^{\prime} F^{(5)}\right)} \text {, } \\
& S_{\mathrm{WZ}}^{(5)}=-\left.\frac{T_{4}}{2} \int \sum_{n=0,2,4} \hat{C}_{n} \wedge \exp \left(\hat{B}_{2}+2 \pi \alpha^{\prime} F_{2}\right)\right|_{4} \text { form } \\
& =\frac{1}{2} \int \mathrm{d}^{5} x \sqrt{-g^{(5)}} \\
& \times\left\{\epsilon ^ { A B C D } \left[\partial _ { A } \Phi ^ { I } \partial _ { B } \Phi ^ { J } \left(\frac{C_{I J} B_{K L}}{4 T_{4}} \partial_{C} \Phi^{K} \partial_{D} \Phi^{L}\right.\right.\right. \\
& +\frac{\pi \alpha^{\prime} C_{I J} F_{C D}}{2}+\frac{C_{0}}{8 T_{4}} B_{I J} B_{K L} \partial_{C} \Phi^{K} \partial_{D} \Phi^{L} \\
& \left.+\frac{\pi \alpha^{\prime} C_{0}}{2} B_{I J} F_{C D}\right)+2 \pi^{2} \alpha^{\prime 2} T_{4} C_{0} F_{A B} F_{C D} \\
& \left.\left.-T_{4}\left(v_{0}+\frac{v_{4}}{\Phi^{4}}\right)\right]\right\} \\
& S_{\mathrm{BSUG}}^{(5)}=\frac{1}{2} \int \mathrm{d}^{5} x \sqrt{-g_{(5)}} e_{(5)} \\
& \times\left[-\frac{M_{5}^{3} R^{(5)}}{2}+\frac{i}{2} \bar{\Psi}_{i \tilde{m}} \Gamma^{\tilde{m} \tilde{n} \tilde{q}} \nabla_{\tilde{n}} \Psi_{\tilde{q}}^{i}-S_{I J} F_{\tilde{m} \tilde{n}}^{I} F^{I \tilde{m} \tilde{n}}\right. \\
& -\frac{1}{2} g_{\alpha \beta}\left(D_{\tilde{m}} \phi^{\mu}\right)\left(D^{\tilde{m}} \phi^{v}\right) \\
& + \text { Fermionic }+ \text { Chern }- \text { Simons }+ \text { Pauli mass }] \text {, }
\end{aligned}
$$

where $T_{(4)}$ is the $\mathrm{D} 4$ brane tension, $\alpha^{\prime}$ is the Regge Slope, $\exp (-\Phi)$ is the closed string dilaton and $C_{0}$ is the Axion. Here $\gamma^{(5)}, B^{(5)}$, and $F^{(5)}$ represent the determinant of the $5 \mathrm{D}$ induced metric $\left(\gamma_{A B}\right)$ and the gauge fields $\left(B_{A B}, F_{A B}\right)$, respectively. Additionally here $v_{0}$ and $v_{4}$ represent the constants characterizing the interaction strength between D4$\bar{D} 4$ brane. In the present context 5-dimensional coordinates $X^{A}=\left(x^{\alpha}, y\right)$, where $y$ parameterizes the extra dimension compactified on the closed interval $[-\pi R,+\pi R]$.

It is useful to introduce the $5 \mathrm{D}$ metric in conformal form

$$
\begin{aligned}
& \mathrm{d} s_{4+1}^{2}=g_{A B} \mathrm{~d} X^{A} \mathrm{~d} X^{B} \\
& =\frac{b_{0}^{2}}{R^{2}\left(\exp (\beta y)+\frac{\Lambda_{(5)} b_{0}^{4}}{24 R^{2}} \exp (-\beta y)\right)} \times\left(\mathrm{d} s_{4}^{2}+R^{2} \beta^{2} \mathrm{~d} y^{2}\right),
\end{aligned}
$$

and $\mathrm{d} s_{4}^{2}=g_{\alpha \beta} \mathrm{d} x^{\alpha} \mathrm{d} x^{\beta}$ is FLRW counterpart. The parameter $\beta$ determines the slope of the warp factor and $R$ represents the compactification radius. Applying dimensional reduction technique via $\mathbf{S}^{\mathbf{1}} / \mathbf{Z}_{\mathbf{2}}$ orbifolding symmetry and using the metric stated in Eq. (2.3) the total effective model for D3 
DBI Galileon in background $\mathcal{N}=1, \mathcal{D}=4$ SUGRA is described by the following action [60]:

$$
\begin{aligned}
S= & \int \mathrm{d}^{4} x \sqrt{-g^{(4)}}\left[\hat{\tilde{K}}(\phi, X)-\tilde{G}(\phi, X) \square^{(4)} \phi+\tilde{l}_{1} R_{(4)}\right. \\
& +\tilde{l}_{4}\left(\mathcal{C}(1) R^{\alpha \beta \gamma \delta(4)} R_{\alpha \beta \gamma \delta}^{(4)}-4 \mathcal{I}(2) R^{\alpha \beta(4)} R_{\alpha \beta}^{(4)}\right. \\
& \left.\left.+\mathcal{A}(6) R_{(4)}^{2}\right)+\tilde{l}_{3}\right],
\end{aligned}
$$

where

$$
\begin{aligned}
& \hat{\tilde{K}}(\phi, X)=-\frac{\tilde{D}}{\tilde{f}(\phi)}\left[\sqrt{1-2 Q X \tilde{f}}-Q_{1}\right] \\
& -\tilde{C}_{5} \tilde{G}(\phi, X)-2 X \tilde{M}\left(T, T^{\dagger}\right)-V(\phi), \\
& \tilde{M}\left(T, T^{\dagger}\right)=\frac{M\left(T, T^{\dagger}\right)}{2 \kappa_{(4)}^{2}}, M\left(T, T^{\dagger}\right)=\frac{\sqrt{2} \beta R^{2}}{\left(T+T^{\dagger}\right)}, \\
& \tilde{D}=\frac{D}{2 \kappa_{(4)}^{2}}, \\
& \tilde{G}(\phi, X)=\left(\frac{\tilde{g}(\phi) k_{1} \tilde{C}_{4}}{\left.2\left(1-2 \tilde{f}(\phi) X k_{2}\right)\right)}\right), \\
& \tilde{g}(\phi)=\tilde{g}_{0}+\tilde{g}_{2} \phi^{2}, \quad \tilde{f}(\phi) \simeq \frac{1}{\left(\tilde{f}_{0}+\tilde{f}_{2} \phi^{2}+\tilde{f}_{4} \phi^{4}\right)} \\
& \tilde{l}_{1}=\left\{\frac{1}{2 \kappa_{(4)}^{2}}\left[1+\frac{\alpha_{(4)}}{R^{2} \beta^{2}}(24 \mathcal{I}(2)-24 \mathcal{A}(9)-16 \mathcal{A}(10))\right]\right. \\
& \left.-\frac{\alpha_{(4)} \mathcal{C}(2)}{\kappa_{(4)}^{2} R^{2} \beta^{2}}\right\}, \quad \tilde{l}_{4}=\frac{\alpha_{(4)}}{2 \kappa_{(4)}^{2}}, \\
& \tilde{l}_{3}=\frac{1}{2 \kappa_{(4)}^{2}}\left[\frac{\alpha_{(4)}}{R^{4} \beta^{4}}(24 \mathcal{C}(4)-144 \mathcal{I}(4)-64 \mathcal{A}(5)+144 \mathcal{A}(7)\right. \\
& \left.+64 \mathcal{A}(8)+192 \mathcal{A}(11))-\frac{3 M_{5}^{3} \beta b_{0}^{6}}{2 \kappa_{(4)}^{2} M_{\mathrm{PL}}^{2} R^{5}} \mathcal{I}(1)\right]
\end{aligned}
$$

where $\alpha_{(4)}, \tilde{l}_{1}, \tilde{l}_{3}, \tilde{l}_{4}$ are effective $4 \mathrm{D}$ couplings and $\kappa_{(4)}$ be the gravitational coupling strength. Here $X$ represents the 4D kinetic term after dimensional reduction given by $X:=-\frac{1}{2} g_{\mu \nu} \partial^{\mu} \phi \partial^{\nu} \phi$. In this context $\left(T, T^{\dagger}\right)$ are the four dimensional background SUGRA moduli fields which are constant after dimensional reduction.

The one-loop corrected Coleman-Weinberg potential is given by [60]

$V(\phi)=\sum_{m=-2, m \neq-1}^{2} C_{2 m}\left[1+D_{2 m} \ln \left(\frac{\phi}{M}\right)\right] \phi^{2 m}$,

where $D_{0}=0$ and the other constants are functions of the effective brane tension for the D3 brane and constant moduli in 4D. Hence using Eq. (2.4) the modified Friedman equation in the presence of effective 4D Gauss-Bonnet coupling can be expressed as [60]:
$H^{4}=\frac{\Lambda_{(4)}+8 \pi G_{(4)} \rho_{\phi}}{\tilde{g}_{1}} \approx \frac{\Lambda_{(4)}+8 \pi G_{(4)} V(\phi)}{\tilde{g}_{1}}$,

where $\rho_{\phi}$ plays the role of energy density of the inflation in 4D effective theory, $\tilde{g}_{1}$ represents the effective 4D GaussBonnet coupling dependent function on FLRW background which can be expressed in terms of the brane tension of $D 3$ brane and $\Lambda_{(4)}$ is the $4 \mathrm{D}$ effective cosmological constant. It is important to note that in the $4 \mathrm{D}$ effective action as stated in Eq. (2.4), the contribution of higher curvature effective Gauss-Bonnet like correction term is dominant compared to Ricci scalar. More precisely one can interpret this to be a non-perturbative solution of the effective field theory where the effective coupling parameter $\tilde{l}_{4}>>\tilde{l}_{1}$. Consequently the effective Friedmann equation in $4 \mathrm{D}$ takes a nontrivial form in the high energy regime, where energy density of the inflaton $\rho_{\phi} \approx V(\phi)>>\tilde{g}_{1}$ of $D 3-\bar{D} 3$ system. Here Eq. (2.7) also implies that within our prescribed setup the non-perturbative regime of effective field theory cannot able to produce the well-known solutions of GR in the low energy limiting situation where $\rho_{\phi} \approx V(\phi)<<\tilde{g}_{1}$. But in the perturbative regime of the effective theory the situation is completely different compared the non-perturbative case. In the regime where the effective coupling parameter $\tilde{l}_{4}<<\tilde{l}_{1}$, it is possible to get back the known solution of GR. In literature it usually identified to be the low energy regime, where the inflaton energy density, $\rho_{\phi} \approx$ $V(\phi)<<\tilde{g}_{1}$ in $D 3-\bar{D} 3$ system. But in the high energy regime, where $\rho_{\phi} \approx V(\phi)>>\tilde{g}_{1}$, it is not possible to realize the essence of the higher curvature terms through Fridemann equations, which will finally control the cosmological dynamics in a nontrivial manner. For more details see Ref. [60], where the Friedmann equations are derived in detail.

\section{Tree level bispectrum analysis}

\subsection{Three-scalar correlation}

To calculate the scalar bispectrum for D3 DBI Galileon we consider here the third-order action up to total derivatives. Using the uniform field gauge analysis the third-order action for three scalar interaction can be written as

$$
\begin{aligned}
S_{\zeta \zeta \zeta}= & \int \mathrm{d} t \mathrm{~d}^{3} x\left\{a^{3} \bar{C}_{1} M_{\mathrm{PL}}^{2} \zeta \dot{\zeta}^{2}\right. \\
& +a \bar{C}_{2} M_{\mathrm{PL}}^{2} \zeta(\partial \zeta)^{2}+a^{3} \bar{C}_{3} M_{\mathrm{PL}} \dot{\zeta}^{3} \\
& +a^{3} \bar{C}_{4} \dot{\zeta}\left(\partial_{i} \zeta\right)\left(\partial_{i} \tilde{\chi}\right)+a^{3}\left(\frac{\bar{C}_{5}}{M_{\mathrm{PL}}^{2}}\right) \partial^{2} \zeta(\partial \tilde{\chi})^{2} \\
& \times a \bar{C}_{6} \dot{\zeta}^{2} \partial^{2} \zeta+\left(\frac{\bar{C}_{7}}{a}\right)\left[\partial^{2} \zeta(\partial \zeta)^{2}-\zeta \partial_{i} \partial_{j}\left(\partial_{i} \zeta\right)\left(\partial_{j} \zeta\right)\right]
\end{aligned}
$$




$$
\begin{aligned}
& +a \frac{\bar{C}_{8}}{M_{\mathrm{PL}}}\left[\partial^{2} \zeta \partial_{i} \zeta \partial_{i} \tilde{\chi}-\zeta \partial_{i} \partial_{j}\left(\partial_{i} \zeta\right)\left(\partial_{j} \tilde{\chi}\right)\right] \\
& \left.+\left.\mathcal{R} \frac{\delta \mathcal{L}_{2}}{\delta \zeta}\right|_{1}\right\}
\end{aligned}
$$

where

$\left.\frac{\delta \mathcal{L}_{2}}{\delta \zeta}\right|_{1}=-2\left[\frac{\mathrm{d}}{\mathrm{d} t}\left(a^{3} Y_{S} \dot{\zeta}\right)-a Y_{S} c_{S}^{2} \partial^{2} \zeta\right]$

can be calculated from the second-order action [60]

$\left(S^{(4)}\right)_{\zeta \zeta}=\int \mathrm{d} t \mathrm{~d}^{3} x a^{3} Y_{S}\left[\dot{\zeta}^{2}-\frac{c_{s}^{2}}{a^{2}}(\partial \zeta)^{2}\right]$.

Here $\bar{C}_{i}(i=1,2,3, \ldots, 8)$ are dimensionless coefficients defined as

$$
\begin{aligned}
& \bar{C}_{1}=\frac{Y_{S}}{M_{\mathrm{PL}}^{2}}\left[3-\frac{L_{1} H}{c_{s}^{2}}\left(3+\frac{\dot{Y}_{S}}{H Y_{S}}\right)+\frac{\mathrm{d}}{\mathrm{d} t}\left(\frac{L_{1}}{c_{s}^{2}}\right)\right], \\
& \bar{C}_{2}=\left[1+\frac{1}{a} \frac{\mathrm{d}}{\mathrm{d} t}\left(a L_{1}\left\{Y_{S}-t_{1}\right\}\right)\right], \\
& \bar{C}_{3}=\frac{L_{1}}{M_{\mathrm{PL}}}\left[L_{1}\left(L_{1} a_{1}+a_{3}\right)+a_{12}+\left(a_{9}+L_{1} a_{4}\right) \frac{Y_{S}}{t_{1}}+\frac{Y_{S}}{c_{s}^{2}}\right], \\
& \bar{C}_{4}=-\frac{Y_{S}}{2 t_{1}}\left\{1+2 t_{1}\left[\frac{\mathrm{d}}{\mathrm{d} t}\left(\frac{A_{5}}{t_{1}^{2}}\right)-\frac{3 H A_{5}}{t_{1}^{2}}\right]\right\}, \\
& \bar{C}_{5}=\frac{M_{\mathrm{PL}}^{2}}{2 t_{1}^{2}}\left[\frac{3 M_{\mathrm{PL}}^{2}}{2}\left(1-H L_{1}\right)\right]-\frac{M_{\mathrm{PL}}^{2}}{2} \frac{\mathrm{d}}{\mathrm{d} t}\left(\frac{A_{5}}{t_{1}^{2}}\right), \\
& \bar{C}_{6}=L_{1}^{2}\left[2 M_{\mathrm{PL}}^{2}-L_{1} a_{4}\right], \\
& \bar{C}_{7}=\frac{L_{1}^{2} M_{\mathrm{PL}}^{2}\left(1-H L_{1}\right)}{6}-\frac{c_{s}^{2} Y_{S} L_{1}^{2} M_{\mathrm{PL}}^{2}}{2 t_{1}}+\frac{M_{\mathrm{PL}}^{2}}{6} \frac{\mathrm{d}}{\mathrm{d} t}\left(L_{1}^{3}\right), \\
& \bar{C}_{8}=M_{\mathrm{PL}}\left\{\frac{L_{1} M_{\mathrm{PL}}^{2}}{t_{1}}\left(H L_{1}-1\right)+\frac{c_{s}^{2} Y_{S} L_{1} M_{\mathrm{PL}}^{2}}{t_{1}^{2}}\right\}
\end{aligned}
$$

and the coefficient of $\left.\frac{\delta \mathcal{L}_{2}}{\delta \zeta}\right|_{1}$ involving spatial and time derivatives in Eq. (3.1) is defined by the following expression:

$$
\begin{aligned}
\mathcal{R}= & \frac{A_{5}}{t_{1}^{2}}\left\{\left(\partial_{k} \zeta\right)\left(\partial_{k} \tilde{\chi}\right)-\partial^{-2} \partial_{i} \partial_{j}\left[\left(\partial_{i} \zeta\right)\left(\partial_{j} \tilde{\chi}\right)\right]\right\} \\
& +p_{1} \zeta \dot{\zeta}-\frac{A_{5} L_{1}}{2 t_{1} a^{2}}\left\{(\partial \zeta)^{2}-\partial^{-2} \partial_{i} \partial_{j}\left[\left(\partial_{i} \zeta\right)\left(\partial_{j} \zeta\right)\right]\right\} .
\end{aligned}
$$

In this context $\mathcal{R} \rightarrow 0$ as $k \rightarrow 0$ at large scale. Additionally

$$
\begin{aligned}
& L_{1}=\left(\frac{M_{\mathrm{PL}}^{2}}{H M_{\mathrm{PL}}^{2}-\dot{\phi} X \tilde{G}_{X}}\right), \tilde{\chi}=\partial^{-2}\left(Y_{S} \dot{\zeta}\right), \quad A_{3}=2 Y_{S}, \\
& A_{5}=-\frac{L_{1} M_{\mathrm{PL}}^{2}}{2}, \\
& Y_{S}=\frac{t_{1}\left(4 t_{1} t_{3}+9 t_{2}^{2}\right)}{3 t_{2}^{2}}, \quad c_{s}^{2}=\frac{3\left(2 H t_{2} t_{1}^{2}-t_{4} t_{2}^{2}-2 t_{1}^{2} \dot{t}_{2}\right)}{t_{1}\left(4 t_{1} t_{3}+9 t_{2}^{2}\right)}, \\
& t_{1}=\tilde{l}_{1}, t_{2}=\left(2 H \tilde{l}_{1}-2 \dot{\phi} X \tilde{G}_{X}\right),
\end{aligned}
$$

$$
\begin{aligned}
t_{3}= & -9 \tilde{l}_{1} H^{2}+3\left(X \hat{\tilde{K}}_{X}+2 X^{2} \hat{\tilde{K}}_{X X}\right) \\
& +18 H \dot{\phi}\left(2 X \tilde{G}_{X}+X^{2} \tilde{G}_{X X}\right), \\
a_{1}= & 3 M_{\mathrm{PL}}^{2} H^{2}-X \hat{\tilde{K}}_{X}-4 X^{2} \hat{\tilde{K}}_{X X} \\
& -\frac{4 X^{3}}{3} X \hat{\tilde{K}}_{X X X}-2 H \dot{\phi}\left(10 X \tilde{G}_{X}+11 X^{2} \tilde{G}_{X X}\right. \\
& \left.+2 X^{3} \tilde{G}_{X X X}\right)+2 X \tilde{G}_{\phi}+\frac{14 X^{2}}{3} \tilde{G}_{\phi X}+\frac{4 X^{3}}{3} \tilde{G}_{\phi X X}, \\
a_{3}= & -3 a_{4}=-3\left[2 M_{\mathrm{PL}}^{2} H-2 \dot{\phi}\left(2 X \tilde{G}_{X}+X^{2} \tilde{G}_{X X}\right)\right], \\
a_{9}= & -\frac{2}{3} a_{12}=-2 M_{\mathrm{PL}}^{2} .
\end{aligned}
$$

It is important to mention here that for scalar and tensor modes ghosts and Laplacian instabilities can be avoided iff $c_{s}^{2}>0, Y_{s}>0$. Throughout the paper we use the required parameters from [60] to compute the bispectrum and trispectrum.

Now following the prescription of the in-in formalism in the interacting picture the three-point correlation function for the quasi-exponential limit, after some trivial algebra, look:

$$
\begin{aligned}
& \left\langle\zeta\left(\vec{k}_{1}\right) \zeta\left(\vec{k}_{2}\right) \zeta\left(\vec{k}_{3}\right)\right\rangle=-i \sum_{j=1}^{8} \int_{-\infty}^{0} \mathrm{~d} \eta a \\
& \quad \times\left\langle 0\left|\left[\zeta\left(\vec{k}_{1}\right) \zeta\left(\vec{k}_{2}\right) \zeta\left(\vec{k}_{3}\right),\left(H_{\mathrm{int}}^{(j)}(\eta)\right)_{\zeta \zeta \zeta}\right]\right| 0\right\rangle \\
& =(2 \pi)^{3} \delta^{(3)}\left(\overrightarrow{k_{1}}+\overrightarrow{k_{2}}+\vec{k}_{3}\right) \mathcal{B}_{\zeta \zeta \zeta}\left(\vec{k}_{1}, \vec{k}_{2}, \vec{k}_{3}\right),
\end{aligned}
$$

where the total Hamiltonian in the interaction picture can be expressed in terms of the third-order Lagrangian density as $\left(H_{\text {int }}(\eta)\right)_{\zeta \zeta \zeta}=\sum_{j=1}^{8}\left(H_{\text {int }}^{(j)}(\eta)\right)_{\zeta \zeta \zeta}=-\int \mathrm{d}^{3} x\left(\mathcal{L}_{3}\right)_{\zeta \zeta \zeta}$.

Throughout this article we use the Bunch-Davies mode function as

$$
\begin{aligned}
& u_{m}(\eta, k)=\frac{\sqrt{-k \eta c_{m}}}{a \sqrt{2 Y_{m}}} \mathcal{H}_{v_{m}}^{(1)}\left(-k \eta c_{m}\right) \\
& \rightarrow \frac{\left(-k c_{m} \eta\right)^{\frac{1}{2}-v_{m}} \exp \left(i\left[v_{m}-\frac{1}{2}\right] \frac{\pi}{2}\right) 2^{v_{m}-\frac{3}{2}}}{2 a \sqrt{Y_{m} c_{m} k}}\left(\frac{\Gamma\left(v_{m}\right)}{\Gamma\left(\frac{3}{2}\right)}\right),
\end{aligned}
$$

with $m=$ ( $S$ [scalar], $T$ [tensor]). Moreover, following the momentum dependent ansatz given in $[45,46,63]$ the bispectrum $\mathcal{B}_{\zeta \zeta \zeta}\left(\vec{k}_{1}, \vec{k}_{2}, \vec{k}_{3}\right)$ is defined as

$$
\mathcal{B}_{\zeta \zeta \zeta}\left(\vec{k}_{1}, \overrightarrow{k_{2}}, \overrightarrow{k_{3}}\right)=\frac{(2 \pi)^{4} \mathcal{P}_{\zeta}^{2}}{\prod_{i=1}^{3} k_{i}^{3}} \mathcal{A}_{\zeta \zeta \zeta}\left(\overrightarrow{k_{1}}, \overrightarrow{k_{2}}, \overrightarrow{k_{3}}\right)=\frac{6}{5} f_{\mathrm{NL} ; 1} P_{\zeta}^{2}
$$

where the symbol ; 1 is used for the three-scalar correlation. Here $\mathcal{A}_{\zeta \zeta \zeta}\left(\overrightarrow{k_{1}}, \vec{k}_{2}, \vec{k}_{3}\right)$ is the shape function for bispectrum and $P_{\zeta}^{2}$ is used for normalization of E-mode polarization expressed in terms of the new combination of the cyclic permutations of two-point correlation functions given by

$$
P_{\zeta}^{2}=P_{\zeta}\left(k_{1}\right) P_{\zeta}\left(k_{2}\right)+P_{\zeta}\left(k_{2}\right) P_{\zeta}\left(k_{3}\right)+P_{\zeta}\left(k_{3}\right) P_{\zeta}\left(k_{1}\right) .
$$


The Power spectra for scalar $\left(P_{\zeta}(k)\right)$ and tensor modes $\left(P_{T}(k)\right)$ at the horizon crossing can be written as

$$
\begin{aligned}
& P_{\zeta}(k)=\left(2^{2 v_{s}-3}\left|\frac{\Gamma\left(v_{s}\right)}{\Gamma\left(\frac{3}{2}\right)}\right|^{2} \frac{\left(1-\epsilon_{V}-s_{V}^{S}\right)^{2} \sqrt{V(\phi)}}{8 \pi^{2} Y_{S} c_{s}^{3} \sqrt{\tilde{g}_{1}} M_{\mathrm{PL}}}\right), \\
& P_{T}(k)=\left(2^{2 v_{T}-3}\left|\frac{\Gamma\left(v_{T}\right)}{\Gamma\left(\frac{3}{2}\right)}\right|^{2} \frac{\left(1-\epsilon_{V}-s_{V}^{T}\right)^{2} \sqrt{V(\phi)}}{2 \pi^{2} Y_{T} c_{T}^{3} \sqrt{\tilde{g}_{1}} M_{\mathrm{PL}}}\right) .
\end{aligned}
$$

Here for the tensor modes we use $\left(P_{T}(k)\right)_{i j ; k l}=\left|u_{h}(\eta, k)\right|^{2}$ $\mathcal{N}_{i j ; k l}, P_{T}(k)=\left(P_{T}(k)\right)_{i j ; i j}$ with the following helicity/spin dependent normalization factor: $\mathcal{N}_{i j ; k l}=\sum_{\lambda} \mathrm{e}_{i j}^{\lambda}(\vec{k}) \mathrm{e}_{k l}^{\dagger(\lambda)}(\vec{k})$.

In this context $f_{\mathrm{NL}}$ represents the nonlinear parameter carrying the signature of primordial non-Gaussianities of the curvature perturbation in bispectrum. The explicit form of $f_{\mathrm{NL}}$ characterizing the bispectrum can be expressed as

$$
\begin{aligned}
f_{\mathrm{NL} ; 1}= & \frac{10}{3 \sum_{i=1}^{3} k_{i}^{3}}\left(\frac{k_{1} k_{2} k_{3}}{2 K^{3}}\right)^{n_{\zeta}-1}\left|\frac{\Gamma\left(v_{s}\right)}{\Gamma\left(\frac{3}{2}\right)}\right|^{2} \\
& \times\left\{\bar{C}_{1}\left[\frac{3}{4} \mathcal{I}_{1}\left(n_{\zeta}-1\right)-\frac{3-\epsilon_{V}}{4 c_{s}^{2}}\left(\frac{1+Y_{S}}{1+\epsilon_{V}}\right)^{2} \mathcal{I}_{1}(\tilde{v})\right]\right. \\
& +\frac{3\left(1-\epsilon_{V}-s_{V}^{S}\right)}{2 Y_{S}}\left[\mathcal{F}_{3} \mathcal{I}_{3}\left(n_{\zeta}-1\right)+\frac{\mathcal{E}_{3}}{c_{s}^{2}} \mathcal{I}_{3}(\tilde{v})\right] \\
& +\frac{\bar{C}_{4}}{8} \mathcal{I}_{4}(\tilde{v})+\frac{\bar{C}_{5} Y_{S}}{4 c_{s}^{2}} \mathcal{I}_{5}(\tilde{v}) \\
& +\frac{3\left(1-\epsilon_{V}-s_{V}^{S}\right)^{2}}{Y_{S}}\left[\mathcal{F}_{6} \mathcal{I}_{6}\left(n_{\zeta}-1\right)+\frac{\mathcal{E}_{6}}{c_{s}^{2}} \mathcal{I}_{6}(\tilde{v})\right] \\
& \left.+\frac{\bar{C}_{7}\left(1-\epsilon_{V}-s_{V}^{S}\right)^{2}}{2 Y_{S} c_{S}^{2}} \mathcal{I}_{7}(\tilde{v})+\frac{\bar{C}_{8}\left(1-\epsilon_{V}-s_{V}^{S}\right)}{8 c_{S}^{2}} \mathcal{I}_{8}(\tilde{v})\right\},
\end{aligned}
$$

where the functional form of the momentum dependent functions $\mathcal{I}_{i}(x) \forall i$ are explicitly mentioned in Appendix B. From the coefficients of $\mathcal{I}_{i}(\tilde{v})$ with $i=1,3,5,7,8$ it seems that the non-Gaussian parameter $f_{\mathrm{NL} ; 1}$ is inverse proportional to the sound speed square for the scalar mode. But these coefficients are not solely characterized by the sound speed for the scalar mode since they depend on other factors like (1) effective Gauss-Bonnet coupling $\left(\alpha_{(4)}\right)$ and (2) higherorder interaction between the graviton and the DBI Galileon in the presence of a quadratic correction of gravity in the Einstein-Hilbert action. Additionally in this context counter terms which appears as the coefficients of $\mathcal{I}_{i}\left(n_{\zeta}-1\right)$ with $i=1,3,6$, and $\mathcal{I}_{4}(\tilde{v})$ originated from the effective GaussBonnet coupling $\left(\alpha_{(4)}\right)$ and higher-order interaction between the graviton (via a Gauss-Bonnet correction) and the DBI Galileon degrees of freedom in the D3 brane in the background of four dimensional $\mathcal{N}=1$ SUGRA multiplet play a very crucial role in this context. In $\alpha_{(4)} \neq 0$ limit such counter terms and dependence on the interaction between the graviton and the higher derivative DBI Galileon cannot be negligible in the slow-roll limit.
Consequently, depending on the signature and the strength of the effective Gauss-Bonnet coupling three situations arise: (1) the counter terms drive other terms, (2) the counter terms and other terms are tuned in such a way that the system is in equilibrium with respect to the sound speed and (3) the sound speed dominated terms win the war.

Here the second situation is not physically interesting and the third situation leads to the trivial feature of the DBI Galileon. Only the nontrivial features comes from the first situation in the context of single field DBI Galileon inflation.

In Eq. (3.12) we have defined $K=k_{1}+k_{2}+k_{3}, x=$ $\left(n_{\zeta}-1, \tilde{v}\right)$, and

$$
\begin{aligned}
& \tilde{v}:=\left(\frac{s_{V}^{S}-2 \epsilon_{V}}{1-\epsilon_{V}-s_{V}^{S}}\right), \quad n_{\zeta}-1=\left(3-2 v_{s}\right)=-\left(\frac{2 \epsilon_{V}+s_{V}^{S}+\delta_{V}}{1-\epsilon_{V}-s_{V}^{S}}\right) \\
& \mathcal{F}_{3}:=-\frac{Y_{S}\left(1+Y_{S}\right)}{1+\epsilon_{V}}\left[1+2 \frac{Y_{S}-\epsilon_{V}+\left(1+Y_{S}\right) \rho_{3}}{1+\epsilon_{V}}+2 \mathcal{T}_{3}\right], \\
& \frac{\dot{\phi} X^{2} \tilde{G}_{X X}}{H}=\left(\rho_{3}+\frac{\rho_{4}}{c_{s}^{2}}\right), \quad \frac{v_{s}}{\Sigma_{G}}:=\left(\mathcal{T}_{3}+\frac{\mathcal{T}_{4}}{c_{s}^{2}}\right), \\
& \mathcal{E}_{3}:=-\frac{Y_{S}\left(1+Y_{S}\right)}{1+\epsilon_{V}}\left[2 \mathcal{T}_{4}-\frac{1+Y_{S}}{1+\epsilon_{V}}\left(1-2 \rho_{4}\right)\right], \\
& \mathcal{F}_{6}:=\frac{2\left(1+Y_{S}\right)^{3}}{\left(1+\epsilon_{V}\right)^{3}}\left[\frac{Y_{S}-\epsilon_{V}}{1+Y_{S}}+\rho_{3}\right], \quad \mathcal{E}_{6}:=\frac{2 \rho_{4}\left(1+Y_{S}\right)^{3}}{\left(1+\epsilon_{V}\right)^{3}}
\end{aligned}
$$

with the four new constants $\rho_{3}, \rho_{4}, \mathcal{T}_{3}, \mathcal{T}_{4}$. In the present context $s_{V}^{S}=\frac{\dot{c}_{s}}{H c_{s}}$ is an extra slow-roll parameter appearing due to the sound speed, $c_{s} \neq 1$ as defined in [60]. For the numerical estimation we have further used the equilateral configuration $\left(k_{1}=k_{2}=k_{3}=k\right.$ and $\left.K=3 k\right)$ in which the nonlinear parameter $f_{\mathrm{NL}}$ can be simplified to the following form:

$$
\begin{aligned}
& f_{\mathrm{NL} ; 1}^{\text {equil }}=\frac{10}{9 k^{3}}\left(\frac{1}{54}\right)^{n_{\zeta}-1}\left|\frac{\Gamma\left(v_{s}\right)}{\Gamma\left(\frac{3}{2}\right)}\right|^{2} \\
& \times\left\{\left(3\left(1-\frac{1}{c_{s}^{2}}\right)-\frac{Y_{S} \delta_{V}}{c_{s}^{2}}+\frac{Y_{S}^{2}}{c_{s}^{2}}-\frac{2 Y_{S} s_{V}^{S}}{c_{s}^{2}}\right)\right. \\
& \times\left[\frac{3}{4} \mathcal{I}_{1}^{\text {equil }}\left(n_{\zeta}-1\right)-\frac{3-\epsilon_{V}}{4 c_{s}^{2}}\left(\frac{1+Y_{S}}{1+\epsilon_{V}}\right)^{2} \mathcal{I}_{1}^{\text {equil }}(\tilde{v})\right] \\
& +\frac{3\left(1-\epsilon_{V}-s_{V}^{S}\right)}{2 Y_{S}}\left[\mathcal{F}_{3} \mathcal{I}_{3}^{\text {equil }}\left(n_{\zeta}-1\right)+\frac{\mathcal{E}_{3}}{c_{S}^{2}} \mathcal{I}_{3}^{\text {equil }}(\tilde{v})\right] \\
& -\frac{1}{8}\left[\frac{Y_{S}}{2}+\frac{Y_{S}}{2}\left(3-Y_{S}\right)\right] \mathcal{I}_{4}^{\text {equil }}(\tilde{v}) \\
& +\frac{Y_{S}}{4 c_{S}^{2}}\left(\frac{4 \epsilon_{V}-Y_{S}\left(3-\epsilon_{V}\right)}{4\left(1+\epsilon_{V}\right)}\right) \mathcal{I}_{5}^{\text {equil }}(\tilde{v}) \\
& +\frac{3\left(1-\epsilon_{V}-s_{V}^{S}\right)^{2}}{Y_{S}}\left[\mathcal{F}_{6} \mathcal{I}_{6}^{\text {equil }}\left(n_{\zeta}-1\right)+\frac{\mathcal{E}_{6}}{c_{s}^{2}} \mathcal{I}_{6}^{\text {equil }}(\tilde{v})\right] \\
& -\frac{\left(1-\epsilon_{V}-s_{V}^{S}\right)^{2}\left(1+Y_{S}\right)^{2}\left(Y_{S}-\epsilon_{V}\right)}{2 Y_{S} c_{S}^{2}\left(1+\epsilon_{V}\right)^{3}} \mathcal{I}_{7}^{\text {equil }}(\tilde{v}) \\
& \left.+\frac{\left(1+Y_{S}\right)\left(Y_{S}-\epsilon_{V}\right)\left(1-\epsilon_{V}-s_{V}^{S}\right)}{4 c_{S}^{2}\left(1+\epsilon_{V}\right)^{2}} \mathcal{I}_{8}^{\text {equil }}(\tilde{\nu})\right\} \text {. }
\end{aligned}
$$


Now using the tensor-to-scalar ratio at the pivot scale $k_{*}$ :

$$
\begin{aligned}
r= & \left(16.2^{2\left(v_{T}-v_{s}\right)}\left|\frac{\Gamma\left(v_{T}\right)}{\Gamma\left(v_{s}\right)}\right|^{2}\left(\frac{1-\epsilon_{V}-s_{V}^{T}}{1-\epsilon_{V}-s_{V}^{S}}\right)^{2} c_{S} \epsilon_{s}\right. \\
& \left.\times\left[1-\frac{3}{2} \mathcal{O}\left(\epsilon_{T}^{2}\right)\right]\right)_{\star}
\end{aligned}
$$

the sound speed $c_{s}$ can be eliminated from Eq. (3.14) also.

Here $s_{V}^{T}=\frac{\dot{c}_{T}}{H c_{T}}$ appears due to the sound speed, $c_{T} \neq 1$. See [60] for the details. The numerical value of $f_{\mathrm{NL} ; 1}^{\text {equil }}$ in the equilateral limit is obtained from our setup as $4<f_{\mathrm{NL} ; 1}^{\text {equil }}<7$ within the window for tensor-to-scalar ratio $0.213<r<$ 0.250 [60]. This is extremely interesting result as it is different from other class of DBI models. The most impressing fact is that the upper bound of $f_{\mathrm{NL} ; 1}^{\text {equil }}$ in the quasi-exponential limit is in good agreement with the combined constraint obtained from the Planck + WMAP9 + high-L + BICEP2 [2-5] data.

\subsection{One-scalar two-tensor correlation}

After applying the gauge fixing condition to uniform gauge the one-scalar and two tensor interaction can be represented by the following third-order action:

$$
\begin{aligned}
S_{\zeta h h}= & \int \mathrm{d} t \mathrm{~d}^{3} x a^{3}\left\{\mathcal{F}_{1} \zeta{\dot{h_{i j}}}^{2}+\frac{\tilde{\mathcal{F}}_{2}}{a^{2}} \zeta h_{i j, k} h_{i j, k}\right. \\
& +\tilde{\mathcal{F}}_{3} \psi_{, k} \dot{h}_{i j} h_{i j, k}+\mathcal{F}_{4} \dot{\zeta} \dot{h}_{i j}^{2}+\frac{\tilde{\mathcal{F}}_{5}}{a^{2}} \partial^{2} \zeta \dot{h}_{i j}^{2} \\
& \left.+\tilde{\mathcal{F}}_{6} \psi_{, i j} \dot{h}_{i k} \dot{h}_{j k}+\frac{\tilde{\mathcal{F}}_{7}}{a^{2}} \zeta_{, i j} \dot{h}_{i k} \dot{h}_{j k}\right\},
\end{aligned}
$$

where the dimensionful coefficients $\mathcal{F}_{i}(i=1,2, \ldots, 7)$ are defined as

$$
\begin{aligned}
\tilde{\mathcal{F}}_{1} & =3 Y_{T}\left[1-\frac{H L_{1} Y_{T}}{c_{T}^{2}}+\frac{Y_{T}}{3} \frac{\mathrm{d}}{\mathrm{d} t}\left(\frac{L_{1}}{c_{T}^{2}}\right)\right], \\
\tilde{\mathcal{F}}_{2} & =Y_{s} c_{s}^{2}, \quad \tilde{\mathcal{F}}_{3}=-2 Y_{s}, \\
\tilde{\mathcal{F}}_{4} & =\frac{L_{1}}{c_{T}^{2}}\left(Y_{T}^{2}-\hat{\tilde{K}}_{X X}\right)+2 \sigma \\
& \times\left[\frac{Y_{s}}{Y_{T}}-1-\frac{H L_{1} Y_{T}}{c_{T}^{2}}\left(6+\frac{\dot{Y}_{s}}{H Y_{s}}\right)\right]+2 Y_{T}^{2} \frac{\mathrm{d}}{\mathrm{d} t}\left(\frac{\sigma L_{1}}{Y_{T} c_{T}^{2}}\right), \\
\tilde{\mathcal{F}}_{5} & =2 \sigma Y_{T} L_{1}\left(\frac{c_{s}^{2}}{c_{T}^{2}}-1\right), \quad \tilde{\mathcal{F}}_{6}=-\frac{4 \sigma Y_{s}}{Y_{T}}, \\
\tilde{\mathcal{F}}_{7} & =4 \sigma Y_{T} L_{1},
\end{aligned}
$$

where we use $\sigma=\dot{\phi} X G_{5 X}$. Now following the prescription of the in-in formalism in the interaction picture three-point one scalar two-tensor correlation function can be expressed in the following form:

$$
\begin{aligned}
& \left\langle\zeta\left(\vec{k}_{1}\right) h_{i j}\left(\vec{k}_{2}\right) h_{k l}\left(\vec{k}_{3}\right)\right\rangle=-i \sum_{q=1}^{7} \int_{-\infty}^{0} \mathrm{~d} \eta a \\
& \times\langle 0|\left[\zeta\left(\vec{k}_{1}\right) h_{i j}\left(\vec{k}_{2}\right) h_{k l}\left(\vec{k}_{3}\right),\left(\left[H_{\mathrm{int}}^{(q)}(\eta)\right]_{i j ; k l}\right)_{\zeta h h]|0\rangle}\right. \\
& =(2 \pi)^{3} \delta^{(3)}\left(\vec{k}_{1}+\vec{k}_{2}+\vec{k}_{3}\right)\left\{B_{\zeta h h}\right\}_{i j ; k l}\left(\vec{k}_{1}, \vec{k}_{2}, \vec{k}_{3}\right),
\end{aligned}
$$

where the total Hamiltonian in the interaction picture can be expressed in terms of the third-order Lagrangian density as $\left(\left[H_{\text {int }}(\eta)\right]_{i j ; k l}\right)_{\zeta h h}=\sum_{q=1}^{7}\left(\left[H_{\text {int }}^{(q)}(\eta)\right]_{i j ; k l}\right)_{\zeta h h}=$ $-\int \mathrm{d}^{3} x\left[\left(\mathcal{L}_{3}\right)_{\zeta h h}\right]_{i j ; k l}$. Moreover, the cross bispectrum $\left\{B_{\zeta h h}\right\}_{i j ; k l}\left(\vec{k}_{1}, \vec{k}_{2}, \vec{k}_{3}\right)$ is defined as

$$
\begin{aligned}
\left\{B_{\zeta h h}\right\}_{i j ; k l}\left(\vec{k}_{1}, \vec{k}_{2}, \vec{k}_{3}\right) & =\frac{(2 \pi)^{4} \mathcal{P}_{u}^{2}}{\prod_{i=1}^{3} k_{i}^{3}}\left(\mathcal{A}_{\zeta h h}\right)_{i j ; k l}\left(\overrightarrow{k_{1}}, \vec{k}_{2}, \vec{k}_{3}\right) \\
& =\frac{6}{5}\left[f_{\mathrm{NL} ; 2}\right]_{i j ; k l}^{u} P_{u}^{2}
\end{aligned}
$$

where the symbol; 2 stands for one scalar two-tensor correlation. Here $\left(\mathcal{A}_{\zeta h h}\right)_{i j ; k l}\left(\vec{k}_{1}, \vec{k}_{2}, \vec{k}_{3}\right)$ is the shape function for bispectrum and the polarization indices are $u=$ 1( $\mathrm{E}-$ mode), 2( $\mathrm{E} \otimes \mathrm{B}-$ mode $), 3(\mathrm{~B}$ - mode). We adopt the following normalization depending on the polarization in which we are interested:

$$
P_{u}^{2}=\left\{\begin{array}{l}
P_{\zeta}\left(k_{1}\right) P_{\zeta}\left(k_{2}\right)+P_{\zeta}\left(k_{2}\right) P_{\zeta}\left(k_{3}\right)+P_{\zeta}\left(k_{3}\right) P_{\zeta}\left(k_{1}\right) \\
: u=1(\mathrm{E}-\text { mode }) \\
P_{\zeta}\left(k_{1}\right) P_{h}\left(k_{2}\right)+P_{\zeta}\left(k_{2}\right) P_{h}\left(k_{3}\right)+P_{\zeta}\left(k_{3}\right) P_{h}\left(k_{1}\right) \\
\quad: u=2(\mathrm{E} \otimes \text { Bmode }) \\
P_{h}\left(k_{1}\right) P_{h}\left(k_{2}\right)+P_{h}\left(k_{2}\right) P_{h}\left(k_{3}\right)+P_{h}\left(k_{3}\right) P_{h}\left(k_{1}\right) \\
\quad: u=3(\mathrm{~B}-\text { mode }) .
\end{array}\right.
$$

Consequently $\left[f_{\mathrm{NL} ; 2}\right]_{i j ; k l}^{u}$ represents the nonlinear parameter which carries the signature of primordial non-Gaussianities of the one-scalar two tensor interaction. The explicit form of $\left[f_{\mathrm{NL} ; 2}\right]_{i j ; k l}^{u}$ characterizing the bispectrum can be calculated as 
Eur. Phys. J. C (2015) 75:241

Page 7 of 28

241

$$
\begin{aligned}
{\left[f_{\mathrm{NL} ; 2}\right]_{i j ; k l}^{u} } & =\frac{10 \mathcal{Q}_{u}^{P O L}}{3 \sum_{i=1}^{3} k_{i}^{3}} \frac{\left(\frac{3}{2}-v_{T}\right)^{2} \underline{K}^{4 v_{T}+2 v_{s}-9}\left[\operatorname{Cos}\left(\left[v_{s}-\frac{1}{2}\right] \frac{\pi}{2}\right)\right]^{\frac{1}{3}}\left[\operatorname{Cos}\left(\left[v_{T}-\frac{1}{2}\right] \frac{\pi}{2}\right)\right]^{\frac{2}{3}}}{c_{S}^{2 v_{s}-3} c_{T}^{4 v_{T}-6}\left(k_{1}\right)^{v_{s}}\left(k_{2} k_{3}\right)^{v_{T}}} \\
& \times\left(2^{2 v_{s}+4 v_{T}-12}\left|\frac{\Gamma\left(v_{s}\right)}{\Gamma\left(\frac{3}{2}\right)}\right|^{2}\left|\frac{\Gamma\left(v_{T}\right)}{\Gamma\left(\frac{3}{2}\right)}\right|^{4} \frac{\left(1-\epsilon_{V}-s_{V}^{S}\right)^{2}\left(1-\epsilon_{V}-s_{V}^{T}\right)^{4} V^{\frac{3}{2}}(\phi)}{Y_{S} Y_{T}^{2} c_{T}^{4} c_{s}^{3} \tilde{g}_{1}^{\frac{3}{2}} M_{\mathrm{PL}}^{3}}\right) \\
& \times\left[32 \tilde{\mathcal{F}}_{1}\left(\nabla_{1}\right)_{i j ; k l}^{u}+4 \tilde{\mathcal{F}}_{2}\left(\nabla_{2}\right)_{i j ; k l}^{u}+2\left(\tilde{\mathcal{F}}_{3}\left(\nabla_{3}\right)_{i j ; k l}^{u}+\tilde{\mathcal{F}}_{4}\left(\nabla_{4}\right)_{i j ; k l}^{u}+\tilde{\mathcal{F}}_{5}\left(\nabla_{5}\right)_{i j ; k l}^{u}+\tilde{\mathcal{F}}_{6}\left(\nabla_{6}\right)_{i j ; k l}^{u}+\tilde{\mathcal{F}}_{7}\left(\nabla_{7}\right)_{i j ; k l}^{u}\right)\right]
\end{aligned}
$$

with polarization index $u=1(\mathrm{E}), 2(\mathrm{E} \otimes \mathrm{B}), 3(\mathrm{~B})$. The functional form of the coefficients $\left(\nabla_{i}\right)_{i j ; k l}^{u} \forall i$ are explicitly mentioned in Appendix B. In this context we define $\underline{K}:=c_{s} k_{1}+c_{T}\left(k_{2}+k_{3}\right)$.

The overall normalization factor for the three types of polarization can be expressed as

$\mathcal{Q}_{u}^{\mathrm{POL}}= \begin{cases}8 & : u=1(\mathrm{E}-\text { mode }) \\ 128 & : u=2(\mathrm{E} \otimes \text { Bode }) \\ 2048: u=3 & (\mathrm{~B}-\text { mode })\end{cases}$

Further, to make the computation simpler without losing any essential information we reduce the complete set in terms of the two-polarization (helicity) mode instead of four complicared tensor indices. For this purpose let us define the reduced physical quantity:

$\bigoplus^{\lambda}(\vec{k})=h_{i j}(\vec{k}) \mathrm{e}_{i j}^{\dagger(\lambda)}$ in terms of which the one-scalar two-tensor correlation is defined as

$$
\begin{aligned}
& \left\langle\zeta\left(\vec{k}_{1}\right) \bigoplus^{\lambda_{2}}\left(\vec{k}_{2}\right) \bigoplus^{\lambda_{3}}\left(\vec{k}_{3}\right)\right\rangle \\
& \quad=(2 \pi)^{3} \delta\left(\vec{k}_{1}+\vec{k}_{2}+\vec{k}_{3}\right) B_{(\zeta h h)}^{\left(\lambda_{1} ; \lambda_{2}\right)}\left(\vec{k}_{1}, \vec{k}_{2}, \vec{k}_{3}\right) .
\end{aligned}
$$

where the cross reduced bispectrum is defined as

$$
\begin{aligned}
B_{(\zeta h h)}^{\left(\lambda_{2} ; \lambda_{3}\right)}\left(\vec{k}_{1}, \vec{k}_{2}, \vec{k}_{3}\right) & =\frac{(2 \pi)^{4} \mathcal{P}_{u}^{2}}{\prod_{i=1}^{3} k_{i}^{3}} \mathcal{A}_{(\zeta h h)}^{\left(\lambda_{2} ; \lambda_{3}\right)} \\
& =\frac{6}{5}\left[f_{\mathrm{NL} ; 2}\right]^{u ;\left(\lambda_{2} ; \lambda_{3}\right)} P_{u}^{2} .
\end{aligned}
$$

Applying the basis transformation the explicit form of $\left[f_{\mathrm{NL} ; 2}\right]^{\left(\lambda_{2} ; \lambda_{3}\right)}$ characterizing the crossed bispectrum can be written as

$$
\begin{aligned}
& {\left[f_{\mathrm{NL} ; 2}\right]^{u ;\left(\lambda_{2} ; \lambda_{3}\right)}=\frac{10 \mathcal{Q}_{u}^{P O L}}{3 \sum_{i=1}^{3} k_{i}^{3}} \frac{\left(\frac{3}{2}-v_{T}\right)^{2} \underline{K}^{4 v_{T}+2 v_{s}-9}\left[\operatorname{Cos}\left(\left[v_{s}-\frac{1}{2}\right] \frac{\pi}{2}\right)\right]^{\frac{1}{3}}\left[\operatorname{Cos}\left(\left[v_{T}-\frac{1}{2}\right] \frac{\pi}{2}\right)\right]^{\frac{2}{3}}}{c_{S}^{2 v_{s}-3} c_{T}^{4 v_{T}-6}\left(k_{1}\right)^{v_{s}}\left(k_{2} k_{3}\right)^{v_{T}}}} \\
& \quad \times\left(2^{2 v_{s}+4 v_{T}-12}\left|\frac{\Gamma\left(v_{s}\right)}{\Gamma\left(\frac{3}{2}\right)}\right|^{2}\left|\frac{\Gamma\left(v_{T}\right)}{\Gamma\left(\frac{3}{2}\right)}\right|^{4} \frac{\left(1-\epsilon_{V}-s_{V}^{S}\right)^{2}\left(1-\epsilon_{V}-s_{V}^{T}\right)^{4} V^{\frac{3}{2}}(\phi)}{Y_{S} Y_{T}^{2} c_{T}^{4} c_{s}^{3} \tilde{g}_{1}^{\frac{3}{2}} M_{\mathrm{PL}}^{3}}\right) \\
& \quad \times\left[32 \tilde{\mathcal{F}}_{1}\left(\nabla_{1}\right)^{u ; \lambda_{2} ; \lambda_{3}}+4 \tilde{\mathcal{F}}_{2}\left(\nabla_{2}\right)^{u ; \lambda_{2} ; \lambda_{3}}+2\left(\tilde{\mathcal{F}}_{3}\left(\nabla_{3}\right)^{u ; \lambda_{2} ; \lambda_{3}}+\tilde{\mathcal{F}}_{4}\left(\nabla_{4}\right)^{u ; \lambda_{2} ; \lambda_{3}}+\tilde{\mathcal{F}}_{5}\left(\nabla_{5}\right)^{u ; \lambda_{2} ; \lambda_{3}}+\tilde{\mathcal{F}}_{6}\left(\nabla_{6}\right)^{u ; \lambda_{2} ; \lambda_{3}}\right.\right. \\
& \left.\left.\quad+\tilde{\mathcal{F}}_{7}\left(\nabla_{7}\right)^{u ; \lambda_{2} ; \lambda_{3}}\right)\right] .
\end{aligned}
$$

Springer 
The functional form of the coefficients $\left(\nabla_{i}\right)^{u ; \lambda_{2} ; \lambda_{3}} \forall i$ after a basis transformation are explicitly mentioned in the Appendix. In the equilateral limit we have

$$
\begin{aligned}
& {\left[f_{\mathrm{NL} ; 2}^{\text {equil }}\right]^{\mathbf{u} ;\left(\lambda_{2} ; \lambda_{3}\right)}=\frac{10 \mathcal{Q}_{u}^{\mathrm{POL}}}{9 k^{3}} \frac{\left(\frac{3}{2}-v_{T}\right)^{2}\left(\left(c_{s}+2 c_{T}\right) k\right)^{4 v_{T}+2 v_{s}-9}\left[\operatorname{Cos}\left(\left[v_{s}-\frac{1}{2}\right] \frac{\pi}{2}\right)\right]^{\frac{1}{3}}\left[\operatorname{Cos}\left(\left[v_{T}-\frac{1}{2}\right] \frac{\pi}{2}\right)\right]^{\frac{2}{3}}}{c_{s}^{2 v_{s}-3} c_{T}^{4 v_{T}-6} k^{v_{s}+2 v_{T}}}} \\
& \quad \times\left(2^{2 v_{s}+4 v_{T}-12}\left|\frac{\Gamma\left(v_{s}\right)}{\Gamma\left(\frac{3}{2}\right)}\right|^{2}\left|\frac{\Gamma\left(v_{T}\right)}{\Gamma\left(\frac{3}{2}\right)}\right|^{4} \frac{\left(1-\epsilon_{V}-s_{V}^{S}\right)^{2}\left(1-\epsilon_{V}-s_{V}^{T}\right)^{4} V^{\frac{3}{2}}(\phi)}{Y_{S} Y_{T}^{2} c_{T}^{4} c_{s}^{3} \tilde{g}_{1}^{\frac{3}{2}} M_{\mathrm{PL}}^{3}}\right)\left[32 \tilde{\mathcal{F}}_{1}\left(\nabla_{1}\right)_{\text {equil }}^{u ; \lambda_{2} ; \lambda_{3}}+4 \tilde{\mathcal{F}}_{2}\left(\nabla_{2}\right)_{\text {equil }}^{u ; \lambda_{2} ; \lambda_{3}}\right. \\
& \left.\quad+2\left(\tilde{\mathcal{F}}_{3}\left(\nabla_{3}\right)_{\text {equil }}^{u ; \lambda_{2} ; \lambda_{3}}+\tilde{\mathcal{F}}_{4}\left(\nabla_{4}\right)_{\text {equil }}^{u ; \lambda_{2} ; \lambda_{3}}+\tilde{\mathcal{F}}_{5}\left(\nabla_{5}\right)_{\text {equil }}^{u ; \lambda_{2} ; \lambda_{3}}+\tilde{\mathcal{F}}_{6}\left(\nabla_{6}\right)_{\text {equil }}^{u ; \lambda_{2} ; \lambda_{3}}+\tilde{\mathcal{F}}_{7}\left(\nabla_{7}\right)_{\text {equil }}^{u ; \lambda_{2} ; \lambda_{3}}\right)\right],
\end{aligned}
$$

where each coefficients and functions are evaluated in equilateral limit.

\subsection{Two-scalar one-tensor correlation}

After gauge fixing the interactions involving one tensor and two scalars are given by the following third-order action:

$$
\begin{aligned}
S_{\zeta \zeta h}=\int & \mathrm{d} t \mathrm{~d}^{3} x a^{3}\left\{\frac{\mathcal{Y}_{1}}{a^{2}} h_{i j} \zeta_{, i} \zeta_{, j}+\frac{\mathcal{Y}_{2}}{a^{2}} \dot{h}_{i j} \zeta_{, i} \zeta_{, j}\right. \\
& +\mathcal{Y}_{3} \dot{h}_{i j} \zeta_{, i} \psi_{, j}+\frac{\mathcal{Y}_{4}}{a^{2}} \partial^{2} h_{i j} \zeta_{, i} \psi_{, j}+\frac{\mathcal{Y}_{5}}{a^{4}} \partial^{2} h_{i j} \zeta_{, i} \zeta_{, j} \\
& \left.+\mathcal{Y}_{6} \partial^{2} h_{i j} \psi_{, i} \psi_{, j}\right\}
\end{aligned}
$$

where the dimensionful coefficients $\mathcal{Y}_{i}(i=1,2, \ldots, 6)$ are defined as

$$
\begin{aligned}
\mathcal{Y}_{1}= & Y_{s} c_{s}^{2}, \\
\mathcal{Y}_{2}= & \frac{L_{1} \hat{\tilde{K}}_{X X}}{4}\left(Y_{s} c_{s}^{2}-Y_{T} c_{T}^{2}\right) \\
& +L_{1} Y_{T}^{2}\left[-\frac{1}{2}+\frac{H L_{1} \hat{\tilde{K}}_{X X}}{4}\left(3+\frac{\dot{Y}_{T}}{H Y_{T}}\right)\right. \\
& \left.-\frac{1}{4} \frac{\mathrm{d}}{\mathrm{d} t}\left(L_{1} \hat{\tilde{K}}_{X X}\right)\right]+\frac{\sigma Y_{s} c_{s}^{2}}{Y_{T}}+2 H L_{1} Y_{T} \sigma \\
& -Y_{T} \frac{\mathrm{d}}{\mathrm{d} t}\left(L_{1} \sigma\right), \\
\mathcal{Y}_{3}= & Y_{S}\left[\frac{3}{2}+\frac{\mathrm{d}}{\mathrm{d} t}\left(\frac{\hat{\tilde{K}}_{X X} L_{1}}{2}+\frac{\sigma}{Y_{T}}\right)\right. \\
& \left.-\left(3 H+\frac{\dot{Y}_{T}}{Y_{T}}\right)\left(\frac{\hat{\tilde{K}}_{X X} L_{1}}{2}+\frac{\sigma}{Y_{T}}\right)\right],
\end{aligned}
$$

$$
\mathcal{Y}_{4}=Y_{S}\left[-\frac{\left(Y_{T}-\hat{\tilde{K}}_{X X} c_{T}^{2}\right) L_{1}}{2}-2 H \sigma L_{1}+\frac{\mathrm{d}}{\mathrm{d} t}\left(L_{1} \sigma\right)\right.
$$

$$
\begin{gathered}
\left.+\frac{\sigma}{Y_{T}^{2}}\left(Y_{T} c_{T}^{2}-Y_{s} c_{s}^{2}\right)\right], \\
\mathcal{Y}_{5}=\frac{Y_{T}^{2} L_{1}}{2}\left[\frac{\left(Y_{T}-\hat{\tilde{K}}_{X X} c_{T}^{2}\right)}{2}+2 H L_{1} \sigma\right. \\
\left.-\frac{\mathrm{d}}{\mathrm{d} t}\left(\sigma L_{1}\right)-\frac{\sigma}{Y_{T}^{2}}\left(3 Y_{T} c_{T}^{2}-Y_{s} c_{s}^{2}\right)\right], \\
\mathcal{Y}_{6}=\frac{Y_{S}^{2}}{4 Y_{T}}\left[1+\frac{6 H \sigma}{Y_{T}}-2 Y_{T} \frac{\mathrm{d}}{\mathrm{d} t}\left(\frac{\sigma}{Y_{T}^{2}}\right)\right] .
\end{gathered}
$$

Following the prescription of the in-in formalism in the interaction picture three-point two scalar one tensor correlation function can be expressed in the following form:

$$
\begin{aligned}
\left\langle\zeta\left(\vec{k}_{1}\right) \zeta\left(\vec{k}_{2}\right) h_{k l}\left(\vec{k}_{3}\right)\right\rangle= & -i \sum_{q=1}^{7} \int_{-\infty}^{0} \mathrm{~d} \eta a \\
& \times\left\langle 0\left|\left[\zeta\left(\vec{k}_{1}\right) \zeta\left(\vec{k}_{2}\right) h_{k l}\left(\vec{k}_{3}\right),\left(\left[H_{\mathrm{int}}^{(q)}(\eta)\right]_{k l}\right)_{\zeta \zeta h}\right]\right| 0\right\rangle \\
& =(2 \pi)^{3} \delta^{(3)}\left(\vec{k}_{1}+\vec{k}_{2}+\vec{k}_{3}\right)\left\{B_{\zeta \zeta h}\right\}_{k l}\left(\vec{k}_{1}, \vec{k}_{2}, \vec{k}_{3}\right),
\end{aligned}
$$

where the total Hamiltonian can be expressed in terms of the third-order Lagrangian density as $\left(\left[H_{\text {int }}(\eta)\right]_{k l}\right)_{\zeta \zeta h}=$ $\sum_{q=1}^{7}\left(\left[H_{\text {int }}^{(q)}(\eta)\right]_{k l}\right)_{\zeta \zeta h}=-\int \mathrm{d}^{3} x\left[\left(\mathcal{L}_{3}\right)_{\zeta \zeta h}\right]_{k l}$. Here the cross bispectrum $\left\{B_{\zeta \zeta h}\right\}_{k l}$ is defined as

$$
\left\{B_{\zeta \zeta h}\right\}_{k l}=\frac{(2 \pi)^{4} \mathcal{P}_{u}^{2}}{\prod_{i=1}^{3} k_{i}^{3}}\left(\mathcal{A}_{\zeta \zeta h}\right)_{k l}=\frac{6}{5}\left[f_{\mathrm{NL} ; 3}\right]_{k l}^{u} P_{u}^{2},
$$

where $\left(\mathcal{A}_{\zeta \zeta h}\right)_{k l}$ is the two scalar one tensor correlation shape function and the symbol ; 3 represents two scalar one tensor correlation. Consequently the nonlinear parameter $\left[f_{\mathrm{NL} ; 3}\right]_{k l}^{u}$ can be expressed as 


$$
\begin{aligned}
& {\left[f_{\mathrm{NL} ; 3}\right]_{k l}^{u}=\frac{10 L_{u}^{\mathrm{POL}} \mathcal{N}_{i j ; k l}}{3 \sum_{i=1}^{3} k_{i}^{3}} \underline{\underline{K}}^{4 v_{s}+2 v_{T}-9}\left[\operatorname{Cos}\left(\left[v_{s}-\frac{1}{2}\right] \frac{\pi}{2}\right)\right]^{\frac{2}{3}}\left[\operatorname{Cos}\left(\left[v_{T}-\frac{1}{2}\right] \frac{\pi}{2}\right)\right]^{\frac{1}{3}}} \\
& c_{s}^{4 v_{s}-6} c_{T}^{2 v_{T}-3}\left(k_{1} k_{2}\right)^{v_{s}} k_{3}^{\nu_{T}} \\
& \quad \times\left(2^{4 v_{s}+2 v_{T}-12}\left|\frac{\Gamma\left(v_{s}\right)}{\Gamma\left(\frac{3}{2}\right)}\right|^{4}\left|\frac{\Gamma\left(v_{T}\right)}{\Gamma\left(\frac{3}{2}\right)}\right|^{2} \frac{\left(1-\epsilon_{V}-s_{V}^{S}\right)^{4}\left(1-\epsilon_{V}-s_{V}^{T}\right)^{2} V^{\frac{3}{2}}(\phi)}{Y_{S}^{2} Y_{T} c_{s}^{6} c_{T}^{3} \tilde{g}_{1}^{\frac{3}{2}} M_{\mathrm{PL}}^{3}}\right)\left(\sum_{v=1}^{6} \mathcal{Y}_{v}\left(\hat{\nabla}_{v}\right)_{i j}\right),
\end{aligned}
$$

where the functional dependence of the coefficients $\left(\hat{\nabla}_{v}\right)_{i j} \forall v$ are explicitly mentioned in Appendix B. In this context $\underline{\underline{K}}:=$ $c_{s}\left(k_{1}+k_{2}\right)+c_{T} k_{3}$.

For the quasi-exponential limit the overall normalization factor for the three types of polarization can be expressed as

$\mathcal{L}_{u}^{\mathrm{POL}}= \begin{cases}1 & : u=1(\mathrm{E}-\text { mode }) \\ 16: u=2(\mathrm{E} \otimes \text { Bmode }) & \\ 256: u=3(\mathrm{~B}-\text { mode }) .\end{cases}$

As mentioned in the previous subsection, performing a basis transformation cross bispectrum for two scalars and one tensor can be expressed as

$$
\begin{aligned}
& \left\langle\zeta\left(\vec{k}_{1}\right) \zeta\left(\vec{k}_{2}\right) \bigoplus^{\lambda}\left(\vec{k}_{3}\right)\right\rangle \\
& \quad=(2 \pi)^{3} \delta^{(3)}\left(\vec{k}_{1}+\vec{k}_{2}+\vec{k}_{3}\right) B_{\lambda}^{(\zeta \zeta h)}\left(\vec{k}_{1}, \vec{k}_{2}, \vec{k}_{3}\right) .
\end{aligned}
$$

where we have used the following parameterization:

$$
B_{\lambda}^{(\zeta \zeta h)}=\frac{(2 \pi)^{4} \mathcal{P}_{u}^{2}}{\prod_{i=1}^{3} k_{i}^{3}} \mathcal{A}_{(\zeta \zeta h)}^{\lambda}=\frac{6}{5}\left[f_{\mathrm{NL} ; 3}\right]^{u ; \lambda} P_{u}^{2} .
$$

The polarized non-Gaussian parameter for two scalar and one tensor mode $\left[f_{\mathrm{NL} ; 3}\right]^{u ; \lambda}$ can be rewritten as

$$
\begin{aligned}
& {\left[f_{\mathrm{NL} ; 3}^{\mathrm{equil}}\right]_{\lambda}^{u}=\frac{20 L_{u}^{\mathrm{POL}} \delta_{\lambda \lambda^{\prime}}}{9 k^{3}}} \\
& \quad \frac{\left(\left(2 c_{s}+c_{T}\right) k\right)^{4 v_{s}+2 v_{T}-9}\left[\operatorname{Cos}\left(\left[v_{s}-\frac{1}{2}\right] \frac{\pi}{2}\right)\right]^{\frac{2}{3}}\left[\operatorname{Cos}\left(\left[v_{T}-\frac{1}{2}\right] \frac{\pi}{2}\right)\right]^{\frac{1}{3}}}{c_{s}^{4 v_{s}-6} c_{T}^{2 v_{T}-3} k^{2 v_{s}+v_{T}}} \\
& \times\left(2^{4 v_{s}+2 v_{T}-12}\left|\frac{\Gamma\left(v_{s}\right)}{\Gamma\left(\frac{3}{2}\right)}\right|^{4}\left|\frac{\Gamma\left(v_{T}\right)}{\Gamma\left(\frac{3}{2}\right)}\right|^{2}\right. \\
& \left.\quad \times \frac{\left(1-\epsilon_{V}-s_{V}^{S}\right)^{4}\left(1-\epsilon_{V}-s_{V}^{T}\right)^{2} V^{\frac{3}{2}}(\phi)}{Y_{S}^{2} Y_{T} c_{S}^{6} c_{T}^{3} \tilde{g}_{1}^{\frac{3}{2}} M_{\mathrm{PL}}^{3}}\right)\left(\sum_{v=1}^{6} \mathcal{Y}_{v}\left(\hat{\nabla}_{v}\right)_{\lambda^{\prime}}^{\text {equil }}\right) .
\end{aligned}
$$

\subsection{Three-tensor correlation}

The interactions involving three tensors are given by the following third-order action:

$$
\begin{aligned}
S_{h h h}= & \int \mathrm{d} t \mathrm{~d}^{3} x a^{3}\left\{\frac{\sigma}{12} \dot{h}_{i j} \dot{h}_{j k} \dot{h}_{k i}+\frac{Y_{T}}{4 a^{2} c_{T}^{2}}\right. \\
& \left.\times\left(h_{i k} h_{j l}-\frac{1}{2} h_{i j} h_{k l}\right) h_{i j, k l}\right\} .
\end{aligned}
$$

where all the coefficients $\left(\hat{\nabla}_{v}\right)_{\lambda^{\prime}} \forall v$ after a basis transformation are explicitly written in Appendix B.

In the equilateral limit the expression for the non-Gaussian parameter $\left(f_{\mathrm{NL}}\right)$ reduces to the following form:
Now following the prescription of the in-in formalism in the interaction picture the three-point three-tensor correlation function can be expressed in the following form: 


$$
\begin{aligned}
& \left\langle h_{i_{1} j_{1}}\left(\vec{k}_{1}\right) h_{i_{2} j_{2}}\left(\vec{k}_{2}\right) h_{i_{3} j_{3}}\left(\vec{k}_{3}\right)\right\rangle \\
& =-i \int_{-\infty}^{0} \mathrm{~d} \eta a\langle 0|\left[h_{i_{1} j_{1}}\left(\vec{k}_{1}\right) h_{i_{2} j_{2}}\left(\vec{k}_{2}\right) h_{i_{3} j_{3}}\left(\vec{k}_{3}\right),\right. \\
& \left.\quad \times\left(\left[H_{\text {int }}(\eta)\right]_{i_{1} j_{1} i_{2} j_{2} i_{3} j_{3}}\right) h h h\right]|0\rangle \\
& =(2 \pi)^{3} \delta^{(3)}\left(\vec{k}_{1}+\vec{k}_{2}+\vec{k}_{3}\right)\left\{B_{h h h}\right\}_{i_{1} j_{1} i_{2} j_{2} i_{3} j_{3}}\left(\vec{k}_{1}, \vec{k}_{2}, \vec{k}_{3}\right),
\end{aligned}
$$

where the total Hamiltonian is expressed in terms of the third-order Lagrangian density as $\left(\left[H_{\text {int }}(\eta)\right]_{i_{1} j_{1} i_{2} j_{2} i_{3} j_{3}}\right)_{h h h}=$ $-\int \mathrm{d}^{3} x\left[\left(\mathcal{L}_{3}\right)_{h h h}\right]_{i_{1} j_{1} i_{2} j_{2} i_{3} j_{3}}$. In this context the bispectrum for the three-tensor correlation can be expressed as

$$
\begin{aligned}
\left\{B_{h h h}\right\}_{i_{1} j_{1} i_{2} j_{2} i_{3} j_{3}}\left(\vec{k}_{1}, \vec{k}_{2}, \vec{k}_{3}\right) & =\frac{(2 \pi)^{4} \mathcal{P}_{u}^{2}}{\prod_{i=1}^{3} k_{i}^{3}} \mathcal{A}_{i_{1} j_{1} i_{2} j_{2} i_{3} j_{3}}^{h h h} \\
& =\frac{6}{5}\left[f_{\mathrm{NL} ; 4}\right] P_{u}^{2},
\end{aligned}
$$

where the symbol ; 4 represents three-tensor correlation. Also, the non-Gaussian parameter is given by

$$
\begin{aligned}
{\left[f_{\mathrm{NL} ; 4}\right]_{i_{1} j_{1} i_{2} j_{2} i_{3} j_{3}}=} & \frac{10 \mathcal{W}_{u}^{\mathrm{POL}}}{3 \sum_{i=1}^{3} k_{i}^{3}} \frac{K^{9-6 v_{T}} \operatorname{Cos}\left(\left[v_{T}-\frac{1}{2}\right] \frac{\pi}{2}\right)}{\left(k_{1} k_{2} k_{3}\right)^{2 v_{T}}} \\
& \times\left(2^{3\left(v_{s}+v_{T}\right)-11}\left|\frac{\Gamma\left(v_{S}\right)}{\Gamma\left(\frac{3}{2}\right)}\right|^{3}\left|\frac{\Gamma\left(v_{T}\right)}{\Gamma\left(\frac{3}{2}\right)}\right|^{3}\right. \\
& \left.\times \frac{\left(1-\epsilon_{V}-s_{V}^{S}\right)^{3}\left(1-\epsilon_{V}-s_{V}^{T}\right)^{3} V^{\frac{3}{2}}(\phi)}{Y_{S}^{\frac{3}{2}} Y_{T}^{\frac{3}{2}} c_{s}^{\frac{9}{2}} c_{T}^{\frac{9}{2}} \tilde{g}_{1}^{\frac{3}{2}} M_{\mathrm{PL}}^{3}}\right) \\
& \times\left(\sum_{p=1}^{3} \Delta_{i_{1} j_{1} i_{2} j_{2} i_{3} j_{3}}^{(p)}\right)
\end{aligned}
$$

where $K=k_{1}+k_{2}+k_{3}$ and the polarization index $u=1(\mathrm{E}-$ mode $), 2(\mathrm{E} \otimes \mathrm{B}-$ mode $), 3(\mathrm{~B}-$ mode $)$. The functional dependence of all the coefficients $\Delta_{i_{1} j_{1} i_{2} j_{2} i_{3} j_{3}}^{(p)} \forall p$ are summarized in Appendix B. For the quasi-exponential limit the overall normalization factor for the three types of polarization can be expressed as

$\mathcal{W}_{u}^{\mathrm{POL}}= \begin{cases}4 & : u=1(\mathrm{E}-\text { mode }) \\ 64 & : u=2(\mathrm{E} \otimes \text { Bmode }) \\ 1024: u=3(\mathrm{~B}-\text { mode }) .\end{cases}$

After performing a basis transformation the relevant threepoint correlation function for the three-tensor interaction can be expressed in terms of the bispectrum as

$$
\begin{aligned}
& \left\langle\bigoplus^{\lambda_{1}}\left(\vec{k}_{1}\right) \bigoplus^{\lambda_{2}}\left(\vec{k}_{2}\right) \bigoplus^{\lambda_{3}}\left(\vec{k}_{3}\right)\right\rangle \\
& =(2 \pi)^{3} \delta^{(3)}\left(\vec{k}_{1}+\vec{k}_{2}+\vec{k}_{3}\right) B_{\lambda_{1}, \lambda_{2}, \lambda_{3}}^{h h h} .
\end{aligned}
$$

where

$$
B_{\lambda_{1}, \lambda_{2}, \lambda_{3}}^{h h h}=\frac{(2 \pi)^{4} \mathcal{P}_{u}^{2}}{\prod_{i=1}^{3} k_{i}^{3}} \mathcal{A}_{(\zeta \zeta h)}^{\lambda_{1}, \lambda_{2}, \lambda_{3}}=\frac{6}{5}\left[f_{\mathrm{NL} ; 4}\right]_{\lambda_{1}, \lambda_{2}, \lambda_{3}}^{u} P_{u}^{2},
$$

where the nonlinear parameter is given by

$$
\begin{aligned}
& {\left[f_{\mathrm{NL} ; 4}\right]_{\lambda_{1}, \lambda_{2}, \lambda_{3}}^{u}=\frac{10 \mathcal{W}_{u}^{\mathrm{POL}}}{3 \sum_{i=1}^{3} k_{i}^{3}} \frac{K^{9-6 v_{T}} \operatorname{Cos}\left(\left[v_{T}-\frac{1}{2}\right] \frac{\pi}{2}\right)}{\left(k_{1} k_{2} k_{3}\right)^{2 v_{T}}}} \\
& \quad \times\left(2^{3\left(v_{s}+v_{T}\right)-11\left|\frac{\Gamma\left(v_{s}\right)}{\Gamma\left(\frac{3}{2}\right)}\right|^{3}\left|\frac{\Gamma\left(v_{T}\right)}{\Gamma\left(\frac{3}{2}\right)}\right|^{3}}\right. \\
& \left.\quad \times \frac{\left(1-\epsilon_{V}-s_{V}^{S}\right)^{3}\left(1-\epsilon_{V}-s_{V}^{T}\right)^{3} V^{\frac{3}{2}}(\phi)}{Y_{S}^{\frac{3}{2}} Y_{T}^{\frac{3}{2}} c_{S}^{\frac{9}{2}} c_{T}^{\frac{9}{2}} \tilde{g}_{1}^{\frac{3}{2}} M_{\mathrm{PL}}^{3}}\right) \\
& \quad \times\left(\sum_{p=1}^{3} \Delta_{\lambda_{1} \lambda_{2} \lambda_{3}}^{(p)}\right) .
\end{aligned}
$$

Once again, all the helicity dependent coefficients $\Delta_{\lambda_{1} \lambda_{2} \lambda_{3}}^{(p)} \forall p$ after a basis transformation are explicitly mentioned in Appendix B.

In the equilateral limit we have

$$
\begin{aligned}
& {\left[f_{\mathrm{NL} ; 4}^{\text {equil }}\right]_{\lambda_{1}, \lambda_{2}, \lambda_{3}}^{u}=\frac{10 \mathcal{W}_{u}^{\mathrm{POL}}(3 k)^{9-6 v_{T}} \operatorname{Cos}\left(\left[v_{T}-\frac{1}{2}\right] \frac{\pi}{2}\right)}{9 k^{3}} \frac{k^{6 v_{T}}}{\left({ }^{3}\right.}} \\
& \times\left(2^{3\left(v_{s}+v_{T}\right)-11}\left|\frac{\Gamma\left(v_{s}\right)}{\Gamma\left(\frac{3}{2}\right)}\right|^{3}\left|\frac{\Gamma\left(v_{T}\right)}{\Gamma\left(\frac{3}{2}\right)}\right|^{3}\right. \\
& \left.\times \frac{\left(1-\epsilon_{V}-s_{V}^{S}\right)^{3}\left(1-\epsilon_{V}-s_{V}^{T}\right)^{3} V^{\frac{3}{2}}(\phi)}{Y_{S}^{\frac{3}{2}} Y_{T}^{\frac{3}{2}} c_{S}^{\frac{9}{2}} c_{T}^{\frac{9}{2}} \tilde{g}_{1}^{\frac{3}{2}} M_{\mathrm{PL}}^{3}}\right) \\
& \times\left(\sum_{p=1}^{3} \Delta_{\lambda_{1} \lambda_{2} \lambda_{3}}^{(p) ; \text { equil }}\right) \text {. }
\end{aligned}
$$

The numerical values of all such non-Gaussian parameters from three-point correlation for different polarizing modes are mentioned in the Table 1 . In this context $\mathbf{P C}$ and $\mathbf{P V}$ stands for the parity conserving and violating contribution for the graviton degrees of freedom.

\section{Tree level trispectrum analysis from four-scalar correlation}

To derive the expression for scalar trispectrum for D3 DBI Galileon let us start from fourth-order action up to total derivatives. Consequently the fourth-order action in the uniform gauge can be expressed as

$S_{\zeta \zeta \zeta \zeta}=S_{\mathbf{C I}}+S_{\mathbf{S E}}+S_{\mathbf{G E}}$

where $S_{\mathbf{C I}}, S_{\mathbf{S E}}$, and $S_{\mathbf{G E}}$ represent the contribution from the contact interaction, scalar exchange and graviton exchange appearing in the four-point correlator. In the next subsections we will discuss the individual contributions separately. 
Table 1 Different non-Gaussian $\left(\left[f_{\mathrm{NL} ; A}\right]^{u ;\left(\lambda_{1} \lambda_{2} \lambda_{3}\right)}\right)$ parameters related to the primordial bispectrum for $A=1$ (three scalar), 2 (one scalar and two tensor), 3 (two scalar and one tensor), 4 (three tensor) with polarization index $u=1(\mathrm{E}-$ mode $), 2(\mathrm{E} \otimes \mathrm{B}-$ mode $), 3(\mathrm{~B}-$ mode $)$ including all helicity degrees of freedom represented by $\lambda_{1}, \lambda_{2}$, and $\lambda_{3}$ esti- mated from our model. In this context "+" and "_" stand for the two projections of helicity for the graviton degrees of freedom and " 0 " represents the helicity for the scalar mode. Here $\mathbf{P C}$ and $\mathbf{P V}$ stand for the parity conserving and violating contributions appearing in the tree level primordial bispectrum analysis

\begin{tabular}{|c|c|c|c|c|c|}
\hline$\left[f_{\mathrm{NL} ; A}\right]^{u ;\left(\lambda_{1} \lambda_{2} \lambda_{3}\right)}($ E-mode $)$ & $\times 10^{-3}$ & {$\left[f_{\mathrm{NL} ; A}\right]^{u ;\left(\lambda_{1} \lambda_{2} \lambda_{3}\right)}(\mathrm{E} \otimes \mathrm{B}$-mode $)$} & $\times 10^{-3}$ & {$\left[f_{\mathrm{NL} ; A}\right]^{u ;\left(\lambda_{1} \lambda_{2} \lambda_{3}\right)}(\mathrm{B}-$ mode $)$} & $\times 10^{-4}$ \\
\hline$\left[f_{\mathrm{NL} ; 1}\right]^{1 ;(000)}(\mathbf{P C})$ & $4000-7000$ & {$\left[f_{\mathrm{NL} ; 1}\right]^{2 ;(000)}(\mathbf{P C})$} & 0 & {$\left[f_{\mathrm{NL} ; 1}\right]^{3 ;(000)}(\mathbf{P C})$} & 0 \\
\hline$\left[f_{\mathrm{NL} ; 2}\right]^{1 ;(0++)}(\mathbf{P V})$ & $3.2-6.7$ & {$\left[f_{\mathrm{NL} ; 2}\right]^{2 ;(0++)}(\mathbf{P V})$} & $2.1-4.5$ & {$\left[f_{\mathrm{NL} ; 2}\right]^{3 ;(0++)}(\mathbf{P V})$} & $2.8-8.7$ \\
\hline$\left[f_{\mathrm{NL} ; 2}\right]^{1 ;(0--)}(\mathbf{P V})$ & $1.4-5.7$ & {$\left[f_{\mathrm{NL} ; 2}\right]^{2 ;(0--)}(\mathbf{P V})$} & $2.1-8.9$ & {$\left[f_{\mathrm{NL} ; 2}\right]^{3 ;(0--)}(\mathbf{P V})$} & $2.7-7.2$ \\
\hline$\left[f_{\mathrm{NL} ; 2}\right]^{1 ;(0+-)}(\mathbf{P V})$ & $2.6-9.6$ & {$\left[f_{\mathrm{NL} ; 2}\right]^{2 ;(0+-)}(\mathbf{P V})$} & $2.9-11.0$ & {$\left[f_{\mathrm{NL} ; 2}\right]^{3 ;(0+-)}(\mathbf{P V})$} & $2.7-8.4$ \\
\hline$\left[f_{\mathrm{NL} ; 2}\right]^{1 ;(0-+)}(\mathbf{P V})$ & $1.7-6.9$ & {$\left[f_{\mathrm{NL} ; 2}\right]^{2 ;(0-+)}(\mathbf{P V})$} & $3.5-7.4$ & {$\left[f_{\mathrm{NL} ; 2}\right]^{3 ;(0-+)}(\mathbf{P V})$} & $1.8-10.6$ \\
\hline$\left[f_{\mathrm{NL} ; 3}\right]^{1 ;(00+)}(\mathbf{P C})$ & $121-432$ & {$\left[f_{\mathrm{NL} ; 3}\right]^{2 ;(00+)}(\mathbf{P C})$} & $78-349$ & {$\left[f_{\mathrm{NL} ; 3}\right]^{3 ;(00+)}(\mathbf{P C})$} & $45-221$ \\
\hline$\left[f_{\mathrm{NL} ; 3}\right]^{1 ;(00-)}(\mathbf{P C})$ & $549-878$ & {$\left[f_{\mathrm{NL} ; 3}\right]^{2 ;(00-)}(\mathbf{P C})$} & $304-883$ & {$\left[f_{\mathrm{NL} ; 3}\right]^{3 ;(00-)}(\mathbf{P C})$} & $189-588$ \\
\hline$\left[f_{\mathrm{NL} ; 4}\right]^{1 ;(+++)}(\mathbf{P V})$ & $0.23-0.97$ & {$\left[f_{\mathrm{NL} ; 4}\right]^{2 ;(+++)}(\mathbf{P V})$} & $0.08-0.32$ & {$\left[f_{\mathrm{NL} ; 4}\right]^{3 ;(+++)}(\mathbf{P V})$} & $0.02-0.34$ \\
\hline$\left[f_{\mathrm{NL} ; 4}\right]^{1 ;(---)}(\mathbf{P V})$ & $0.06-0.41$ & {$\left[f_{\mathrm{NL} ; 4}\right]^{2 ;(---)}(\mathbf{P V})$} & $0.09-0.67$ & {$\left[f_{\mathrm{NL} ; 4}\right]^{3 ;(---)}(\mathbf{P V})$} & $0.23-1.7$ \\
\hline$\left[f_{\mathrm{NL} ; 4}\right]^{1 ;(++-)}(\mathbf{P V})$ & $0.23-0.93$ & {$\left[f_{\mathrm{NL} ; 4}\right]^{2 ;(++-)}(\mathbf{P V})$} & $0.18-0.67$ & {$\left[f_{\mathrm{NL} ; 4}\right]^{3 ;(++-)}(\mathbf{P V})$} & $0.03-0.53$ \\
\hline$\left[f_{\mathrm{NL} ; 4}\right]^{1 ;(+--)}(\mathbf{P V})$ & $0.01-0.35$ & {$\left[f_{\mathrm{NL} ; 4}\right]^{2 ;(+--)}(\mathbf{P V})$} & $0.07-0.44$ & {$\left[f_{\mathrm{NL} ; 4}\right]^{3 ;(+--)}(\mathbf{P V})$} & $0.02-0.42$ \\
\hline$\left[f_{\mathrm{NL} ; 4}\right]^{1 ;(-+-)}(\mathbf{P V})$ & $0.04-0.39$ & {$\left[f_{\mathrm{NL} ; 4}\right]^{2 ;(-+-)}(\mathbf{P V})$} & $0.02-0.32$ & {$\left[f_{\mathrm{NL} ; 4}\right]^{3 ;(-+-)}(\mathbf{P V})$} & $0.09-0.51$ \\
\hline$\left[f_{\mathrm{NL} ; 4}\right]^{1 ;(-++)}(\mathbf{P V})$ & $0.03-0.56$ & {$\left[f_{\mathrm{NL} ; 4}\right]^{2 ;(-++)}(\mathbf{P V})$} & $0.1-0.43$ & {$\left[f_{\mathrm{NL} ; 4}\right]^{3 ;(-++)}(\mathbf{P V})$} & $0.17-0.63$ \\
\hline$\left[f_{\mathrm{NL} ; 4}\right]^{1 ;(--+)}(\mathbf{P V})$ & $0.09-0.34$ & {$\left[f_{\mathrm{NL} ; 4}\right]^{2 ;(--+)}(\mathbf{P V})$} & $0.07-0.41$ & {$\left[f_{\mathrm{NL} ; 4}\right]^{3 ;(--+)}(\mathbf{P V})$} & $0.05-0.44$ \\
\hline
\end{tabular}

\subsection{Contact interaction}

Taking into account the contribution coming from contact interaction of effective DBI Galileon in the fourth-order action in uniform gauge we get

$S_{\mathbf{C I}}=\int \mathrm{d} t \mathrm{~d}^{3} x \frac{a^{3}}{4}\left\{\overline{\mathcal{U}}_{1} \dot{\zeta}^{4}-\frac{(\partial \zeta)^{2}}{a^{2}} \dot{\zeta}^{2} \overline{\mathcal{U}}_{2}+\overline{\mathcal{U}}_{3} \frac{(\partial \zeta)^{4}}{a^{4}}\right\}$,

where the coefficients $\overline{\mathcal{U}}_{i}(i=1,2,3)$ for the effective DBI Galileon are defined as

$$
\begin{aligned}
\overline{\mathcal{U}}_{1}= & \left(\frac{\dot{\phi}^{4}}{6}\left[\hat{\tilde{K}}_{4 X}-\tilde{G}_{4 X} \dot{\phi}^{2}\right]+\dot{\phi}^{2}\left[\hat{\tilde{K}}_{X X X}-\tilde{G}_{X X X} \dot{\phi}^{2}\right]\right. \\
& \left.+\frac{1}{2}\left[\hat{\tilde{K}}_{X X}-\tilde{G}_{X X} \dot{\phi}^{2}\right]\right), \\
\overline{\mathcal{U}}_{2}= & \left(\dot{\phi}^{2}\left[\hat{\tilde{K}}_{X X X}-\tilde{G}_{X X X} \dot{\phi}^{2}\right]+\hat{\tilde{K}}_{X X}-\tilde{G}_{X X} \dot{\phi}^{2}\right), \\
\overline{\mathcal{U}}_{3}= & \frac{1}{2}\left[\hat{\tilde{K}}_{X X}-\tilde{G}_{X X} \dot{\phi}^{2}\right],
\end{aligned}
$$

where $\hat{\tilde{K}}(\phi, X)$ and $\tilde{G}(\phi, X)$ are explicitly mentioned in Eq. (2.5). Using the in-in procedure the four-point correlator function for quasi-exponential situation can be expressed in the following form:

$$
\begin{aligned}
& \left\langle\zeta\left(\overrightarrow{k_{1}}\right) \zeta\left(\overrightarrow{k_{2}}\right) \zeta\left(\overrightarrow{k_{3}}\right) \zeta\left(\vec{k}_{4}\right)\right\rangle=-i \sum_{j=1}^{\mathbf{C I}} \int_{-\infty}^{0} \mathrm{~d} \eta a \\
& \quad \times\left\langle 0\left|\left[\zeta\left(\overrightarrow{k_{1}}\right) \zeta\left(\vec{k}_{2}\right) \zeta\left(\vec{k}_{3}\right) \zeta\left(\vec{k}_{4}\right),\left(H_{\mathrm{int}}^{(j)}(\eta)\right)_{\zeta \zeta \zeta \zeta}^{\mathbf{C I}}\right]\right| 0\right\rangle \\
& =(2 \pi)^{3} \delta^{(3)}\left(\vec{k}_{1}+\vec{k}_{2}+\vec{k}_{3}+\vec{k}_{4}\right) \mathcal{T}_{\zeta}^{\mathbf{C I}}\left(\vec{k}_{1}, \vec{k}_{2}, \vec{k}_{3}, \vec{k}_{4}\right)
\end{aligned}
$$

where in the interaction picture the Hamiltonian can be written as $\left(H_{\text {int }}(\eta)\right)_{\zeta \zeta \zeta \zeta}^{\mathbf{C I}}=\sum_{j=1}^{3}\left(H_{\text {int }}^{(j)}(\eta)\right)_{\zeta \zeta \zeta \zeta}^{\mathbf{C I}}$.

Here following the ansatz used in [22] the trispectrum $\mathcal{T}_{\zeta}^{\mathbf{C I}}\left(\overrightarrow{k_{1}}, \overrightarrow{k_{2}}, \overrightarrow{k_{3}}, \overrightarrow{k_{4}}\right)$ for contact interaction is defined as

$$
\begin{aligned}
& \mathcal{T}_{\zeta}^{\mathbf{C I}}\left(\overrightarrow{k_{1}}, \overrightarrow{k_{2}}, \vec{k}_{3}, \vec{k}_{4}\right)=\frac{1}{\prod_{i=1}^{4} k_{i}^{3}} \\
& \quad \times\left[\left(k_{1}^{3} k_{2}^{3}+k_{3}^{3} k_{4}^{3}\right)\left(k_{13}^{-3}+k_{14}^{-3}\right)+\left(k_{1}^{3} k_{4}^{3}+k_{2}^{3} k_{3}^{3}\right)\left(k_{12}^{-3}+k_{13}^{-3}\right)\right. \\
& \left.\quad+\left(k_{1}^{3} k_{3}^{3}+k_{2}^{3} k_{4}^{3}\right)\left(k_{12}^{-3}+k_{14}^{-3}\right)\right]\left\{\tau_{\mathrm{NL}}^{\mathbf{C I}} P_{\zeta(1)}^{3}+\frac{54}{25} g_{\mathrm{NL}}^{\mathbf{C I}} P_{\zeta(2)}^{3}\right\},
\end{aligned}
$$

where

$$
\begin{aligned}
& P_{\zeta(1)}^{3}=\sum_{j<p, i \neq j, p} P_{\zeta}\left(k_{i j}\right) P_{\zeta}\left(k_{j}\right) P_{\zeta}\left(k_{p}\right), \\
& P_{\zeta(2)}^{3}=\sum_{i<j<p} P_{\zeta}\left(k_{i}\right) P_{\zeta}\left(k_{j}\right) P_{\zeta}\left(k_{p}\right),
\end{aligned}
$$

such that 


$$
\begin{aligned}
P_{\zeta}^{3}= & P_{\zeta(1)}^{3}+\frac{3456}{25} \frac{1}{\bar{K}^{3}}\left[\left(k_{1}^{3} k_{2}^{3}+k_{3}^{3} k_{4}^{3}\right)\left(k_{13}^{-3}+k_{14}^{-3}\right)\right. \\
& +\left(k_{1}^{3} k_{4}^{3}+k_{2}^{3} k_{3}^{3}\right)\left(k_{12}^{-3}+k_{13}^{-3}\right) \\
& \left.+\left(k_{1}^{3} k_{3}^{3}+k_{2}^{3} k_{4}^{3}\right)\left(k_{12}^{-3}+k_{14}^{-3}\right)\right] P_{\zeta(2)}^{3},
\end{aligned}
$$

and $\tau_{\mathrm{NL}}^{\mathrm{CI}}$ and $g_{\mathrm{NL}}^{\mathrm{CI}}$ are the two nonlinear parameters which carry the signatures of primordial non-Gaussianities of the curvature perturbation in the trispectrum analysis. By knowing $\tau_{\mathrm{NL}}^{\mathbf{C I}}$ the other parameter $g_{\mathrm{NL}}^{\mathbf{C I}}$ can be calculated by making use of the following relation [65]:

$$
\begin{aligned}
g_{\mathrm{NL}}^{\mathbf{C I}}= & \frac{64}{\bar{K}^{3}}\left[\left(k_{1}^{3} k_{2}^{3}+k_{3}^{3} k_{4}^{3}\right)\left(k_{13}^{-3}+k_{14}^{-3}\right)\right. \\
& +\left(k_{1}^{3} k_{4}^{3}+k_{2}^{3} k_{3}^{3}\right)\left(k_{12}^{-3}+k_{13}^{-3}\right)+\left(k_{1}^{3} k_{3}^{3}\right. \\
& \left.\left.+k_{2}^{3} k_{4}^{3}\right)\left(k_{12}^{-3}+k_{14}^{-3}\right)\right] \tau_{\mathrm{NL}}^{\mathbf{C I}},
\end{aligned}
$$

where $\bar{K}=k_{1}+k_{2}+k_{3}+k_{4}$. Therefore, there is only one independent piece of information, namely $\tau_{\mathrm{NL}}^{\mathrm{CI}}$, which carries information as regards the trispectrum obtained from the contact interaction.

To proceed, we denote the angle between $\vec{k}_{i}$ and $\vec{k}_{j}$ (with $i \neq j$ ) by $\Theta_{i j}$; then

$$
\begin{aligned}
& \operatorname{Cos}\left(\Theta_{12}\right)=\operatorname{Cos}\left(\Theta_{34}\right):=\operatorname{Cos}\left(\Theta_{3}\right), \\
& \operatorname{Cos}\left(\Theta_{23}\right)=\operatorname{Cos}\left(\Theta_{14}\right):=\operatorname{Cos}\left(\Theta_{1}\right), \\
& \operatorname{Cos}\left(\Theta_{13}\right)=\operatorname{Cos}\left(\Theta_{24}\right):=\operatorname{Cos}\left(\Theta_{2}\right)
\end{aligned}
$$

subject to the constraint

$\operatorname{Cos}\left(\Theta_{1}\right)+\operatorname{Cos}\left(\Theta_{2}\right)+\operatorname{Cos}\left(\Theta_{3}\right)=-1$ comes from the conservation of momentum. Additionally we have used

$$
\begin{aligned}
k_{14} & =k_{23}=\left|\vec{k}_{1}+\vec{k}_{4}\right|=\left|\vec{k}_{2}+\vec{k}_{3}\right| \\
& =\sqrt{k_{1}^{2}+k_{4}^{2}+2 k_{1} k_{4} \operatorname{Cos}\left(\Theta_{1}\right)} \\
& =\sqrt{k_{2}^{2}+k_{3}^{2}+2 k_{2} k_{3} \operatorname{Cos}\left(\Theta_{1}\right)}, \\
k_{24} & =k_{13}=\left|\overrightarrow{k_{2}}+\vec{k}_{4}\right|=\left|\vec{k}_{1}+\vec{k}_{3}\right| \\
& =\sqrt{k_{2}^{2}+k_{4}^{2}+2 k_{2} k_{4} \operatorname{Cos}\left(\Theta_{2}\right)} \\
& =\sqrt{k_{1}^{2}+k_{3}^{2}+2 k_{1} k_{3} \operatorname{Cos}\left(\Theta_{2}\right),} \\
k_{34} & =k_{12}=\left|\overrightarrow{k_{3}}+\vec{k}_{4}\right|=\left|\vec{k}_{1}+\vec{k}_{2}\right| \\
& =\sqrt{k_{3}^{2}+k_{4}^{2}+2 k_{3} k_{4} \operatorname{Cos}\left(\Theta_{3}\right)} \\
& =\sqrt{k_{1}^{2}+k_{2}^{2}+2 k_{1} k_{2} \operatorname{Cos}\left(\Theta_{3}\right) .}
\end{aligned}
$$

The explicit form of $\tau_{\mathrm{NL}}^{\mathrm{CI}}$ characterizing the trispectrum obtained from the contact interaction can be expressed for our model as

$$
\begin{aligned}
& \tau_{\mathrm{NL}}^{\mathrm{CI}} \\
& =\frac{2^{8 v_{s}-6} \pi^{6} \operatorname{Cos}\left(\left[v_{s}-\frac{1}{2}\right] \frac{\pi}{2}\right)}{\left[\left(k_{1}^{3} k_{2}^{3}+k_{3}^{3} k_{4}^{3}\right)\left(k_{13}^{-3}+k_{14}^{-3}\right)+\left(k_{1}^{3} k_{4}^{3}+k_{2}^{3} k_{3}^{3}\right)\left(k_{12}^{-3}+k_{13}^{-3}\right)+\left(k_{1}^{3} k_{3}^{3}+k_{2}^{3} k_{4}^{3}\right)\left(k_{12}^{-3}+k_{14}^{-3}\right)\right]} \\
& \times\left|\frac{\Gamma\left(v_{s}\right)}{\Gamma\left(\frac{3}{2}\right)}\right|^{8} \frac{\left(1-\epsilon_{V}-s_{V}^{S}\right)^{8} H^{8}}{Y_{S}^{4} c_{s}^{12}\left(k_{1} k_{2} k_{3} k_{4}\right)^{2 v_{s}}}\left\{\frac { 8 \overline { \mathcal { U } } _ { 1 } \overline { K } ^ { 8 v _ { s } - 5 } } { 1 3 } \left[\Gamma\left(17-8 v_{s}\right)\right.\right. \\
& \times \bar{K}^{8} G_{1}-i \Gamma\left(16-8 v_{s}\right) \bar{K}^{7} G_{2} \\
& +\Gamma\left(15-8 v_{s}\right) \bar{K}^{6} G_{3}-i \Gamma\left(14-8 v_{s}\right) \bar{K}^{5} G_{4} \\
& +\Gamma\left(13-8 v_{s}\right) \bar{K}^{4} G_{5}-i \Gamma\left(12-8 v_{s}\right) \bar{K}^{3} G_{6} \\
& \left.+\Gamma\left(11-8 v_{s}\right) \bar{K}^{2} G_{7}-i \Gamma\left(10-8 v_{s}\right) \bar{K} G_{8}+G_{9}\right] \\
& +\frac{\overline{\mathcal{U}}_{2} \bar{K}^{8 v_{s}-3}}{32}\left[\left(\vec{k}_{3} \cdot \vec{k}_{4}\right) \overline{\mathcal{I}}(3,4 ; 1,2)+\left(\vec{k}_{2} \cdot \vec{k}_{4}\right) \overline{\mathcal{I}}(2,4 ; 1,3)\right. \\
& +\left(\vec{k}_{2} . k_{3}\right) \overline{\mathcal{I}}(2,3 ; 1,4)+\left(\vec{k}_{1} \cdot \vec{k}_{4}\right) \overline{\mathcal{I}}(1,4 ; 2,3) \\
& \left.+\left(\vec{k}_{1} \cdot \vec{k}_{2}\right) \overline{\mathcal{I}}(1,2 ; 3,4)+\left(\vec{k}_{1} \cdot \vec{k}_{3}\right) \overline{\mathcal{I}}(1,3 ; 2,4)\right] \\
& +\frac{\overrightarrow{\mathcal{U}}_{3} \bar{K}^{8 v_{s}+12}}{8}\left[\left(\overrightarrow{k_{1}} \cdot \overrightarrow{k_{2}}\right)\left(\overrightarrow{k_{3}} \cdot \vec{k}_{4}\right)+\left(\overrightarrow{k_{1}} \cdot \overrightarrow{k_{3}}\right)\left(\overrightarrow{k_{2}} \cdot \overrightarrow{k_{4}}\right)\right. \\
& \left.+\left(\vec{k}_{1} \cdot \vec{k}_{4}\right)\left(\vec{k}_{2} \cdot \vec{k}_{3}\right)\right]\left(\frac{\overline{\mathcal{Z}}_{1} \Gamma\left(13-8 v_{s}\right)}{(\bar{K})^{13}}+\frac{\overline{\mathcal{Z}}_{2} \Gamma\left(14-8 v_{s}\right)}{(\bar{K})^{14}}\right. \\
& \left.\left.-\frac{\overline{\mathcal{Z}}_{3} \Gamma\left(15-8 v_{s}\right)}{(\bar{K})^{15}}-\frac{\overline{\mathcal{Z}}_{4} \Gamma\left(16-8 v_{s}\right)}{(\bar{K})^{16}}+\frac{\overline{\mathcal{Z}}_{5} \Gamma\left(17-8 v_{s}\right)}{(\bar{K})^{17}}\right)\right\}
\end{aligned}
$$

where the functional dependence of the momentum dependent functions $G_{i} \forall i, \mathcal{Z}_{q} \forall q$ and $\overline{\mathcal{I}}(i, j ; m, n)$ are given in Appendix B. It is important to mention here that the $4 \mathrm{D}$ effective coupling and the interaction between the higher order graviton and the DBI Galileon plays a significant role in the slow-roll regime. From Eq. (4.11) it is evident that the nonGaussian parameter $\tau_{\mathrm{NL}}^{\mathrm{CI}}$ obtained from the contact interaction is inversely proportional to the 12th power of the sound speed for the scalar mode. But depending on the signature and strength of the Gauss-Bonnet coupling the behavior of the $\tau_{\mathrm{NL}}^{\mathrm{CI}}$ changes.

Further, using the equilateral configuration $\left(k_{1}=k_{2}=\right.$ $k_{3}=k_{4}=k$ and $\left.\bar{K}=4 k\right)$ and incorporating the contribution from the maximum shape of the trispectrum $\left(\operatorname{Cos}\left(\Theta_{1}\right)=\right.$ $\operatorname{Cos}\left(\Theta_{2}\right)=\operatorname{Cos}\left(\Theta_{3}\right)=-\frac{1}{3}$ and $k_{i j}($ for $\left.i<j)=\frac{2 k}{\sqrt{3}}\right)$ the nonlinear parameter can be expressed as

$$
\begin{aligned}
& \tau_{\mathrm{NL}}^{\text {equil; CI }}=\frac{2^{24 v_{s}-5} \pi^{6} \operatorname{Cos}\left(\left[v_{s}-\frac{1}{2}\right] \frac{\pi}{2}\right)}{9 \sqrt{3} k^{3}}\left|\frac{\Gamma\left(v_{s}\right)}{\Gamma\left(\frac{3}{2}\right)}\right|^{8} \\
& \quad \times \frac{\left(1-\epsilon_{V}-s_{V}^{S}\right)^{8} H^{8}}{Y_{S}^{4} c_{s}^{12}}\left\{\frac { 8 \overline { \mathcal { U } } _ { 1 } } { 1 3 3 1 2 k ^ { 5 } } \left[65536 \Gamma\left(17-8 v_{s}\right) k^{8} G_{1}^{\text {equil }}\right.\right. \\
& \quad-16384 i \Gamma\left(16-8 v_{s}\right) k^{7} G_{2}^{\text {equil }}+4096 \Gamma\left(15-8 v_{s}\right) k^{6} G_{3}^{\text {equil }} \\
& \quad-1024 i \Gamma\left(14-8 v_{s}\right) k^{5} G_{4}^{\text {equil }} \\
& +256 \Gamma\left(13-8 v_{s}\right) k^{4} G_{5}^{\text {equil }}-64 i \Gamma\left(12-8 v_{s}\right) k^{3} G_{6}^{\text {equil }} \\
& +16 \Gamma\left(11-8 v_{s}\right) k^{2} G_{7}^{\text {equil }} \\
& \left.\quad-4 i \Gamma\left(10-8 v_{s}\right) k G_{8}^{\text {equil }}+G_{9}^{\text {equil }}\right]-\frac{\overline{\mathcal{U}}_{2}}{1024 k} \overline{\mathcal{I}}^{\text {equil }}
\end{aligned}
$$




$$
\begin{aligned}
& +\frac{838861 \overline{\mathcal{U}}_{3}}{12}\left(\frac{\overline{\mathcal{Z}}_{1}^{\text {equil }} k^{3} \Gamma\left(13-8 v_{s}\right)}{67108864}\right. \\
& +\frac{\overline{\mathcal{Z}}_{2}^{\text {equil }} k^{2} \Gamma\left(14-8 v_{s}\right)}{268435456}-\frac{\overline{\mathcal{Z}}_{3}^{\text {equil }} k \Gamma\left(15-8 v_{s}\right)}{1073741824} \\
& \left.\left.-\frac{\overline{\mathcal{Z}}_{4}^{\text {equil }} \Gamma\left(16-8 v_{s}\right)}{4294967296}+\frac{\overline{\mathcal{Z}}_{5}^{\text {equil }} \Gamma\left(17-8 v_{s}\right)}{17179869184}\right)\right\} .
\end{aligned}
$$

\subsection{Scalar exchange}

Within the in-in picture formalism, to calculate the four-point correlation function resulting from a correlation established via the scalar exchange mode of effective DBI Galileon we start with the following action in the uniform gauge:

$$
S_{\mathbf{S E}}=\int \mathrm{d} t \mathrm{~d}^{3} x a^{3}\left\{\mathbf{A} \dot{\zeta}^{3}-\frac{(\partial \zeta)^{2}}{a^{2}} \dot{\zeta} \mathbf{B}\right\},
$$

where the coefficients $(\mathbf{A}, \mathbf{B})$ are defined as

$$
\begin{aligned}
& \mathbf{A}=\left(\frac{\dot{\phi}}{2}\left[\hat{\tilde{K}}_{X X}-\tilde{G}_{X X}\right]+\frac{\dot{\phi}^{3}}{6}\left[\hat{\tilde{K}}_{X X X}-\tilde{G}_{X X X} \dot{\phi}^{2}\right]\right), \\
& \mathbf{B}=-\frac{\dot{\phi}}{2}\left[\hat{\tilde{K}}_{X X}-\tilde{G}_{X X} \dot{\phi}^{2}\right] .
\end{aligned}
$$

Using the in-in procedure the four-point correlator function for the quasi-exponential limit can be expressed in the following form:

$$
\begin{aligned}
& \left\langle\zeta\left(\vec{k}_{1}\right) \zeta\left(\vec{k}_{2}\right) \zeta\left(\vec{k}_{3}\right) \zeta\left(\vec{k}_{4}\right)\right\rangle^{\mathbf{S E}}=-i \sum_{j=1}^{2} \sum_{p=1}^{2} \int_{-\infty}^{0} \mathrm{~d} \eta \int_{-\infty}^{\eta} \mathrm{d} \tilde{\eta} \\
& \quad \times\left\langle 0\left|\left[\left[\zeta\left(\vec{k}_{1}\right) \zeta\left(\vec{k}_{2}\right) \zeta\left(\vec{k}_{3}\right) \zeta\left(\vec{k}_{4}\right),\left(H_{\mathrm{int}}^{(j)}(\eta)\right)_{\zeta \zeta \zeta}^{\mathbf{S E}}\right],\left(H_{\mathrm{int}}^{(p)}(\tilde{\eta})\right)_{\zeta \zeta \zeta}^{\mathbf{S E}}\right]\right| 0\right\rangle \\
& \quad=(2 \pi)^{3} \delta^{(3)}\left(\vec{k}_{1}+\vec{k}_{2}+\vec{k}_{3}+\vec{k}_{4}\right) \mathcal{T}_{\zeta}^{\mathbf{S E}}\left(\vec{k}_{1}, \vec{k}_{2}, \vec{k}_{3}, \vec{k}_{4}\right),
\end{aligned}
$$

where in the interaction picture the Hamiltonian can be written in terms of the third-order Lagrangian density as $\left(H_{\text {int }}(\eta)\right)_{\zeta \zeta \zeta}^{\mathbf{S E}}=\sum_{j=1}^{2}\left(H_{\text {int }}^{(j)}(\eta)\right)_{\zeta \zeta \zeta}^{\mathbf{S E}}=-\int \mathrm{d}^{3} x \mathcal{L}_{3}^{\mathbf{S E}}$. Hence following the ansatz used in [22] the trispectrum $\mathcal{T}_{\zeta}^{\mathbf{S E}}\left(\overrightarrow{k_{1}}, \overrightarrow{k_{2}}, \overrightarrow{k_{3}}, \overrightarrow{k_{4}}\right)$ is defined as

$$
\begin{aligned}
\mathcal{T}_{\zeta}^{\mathbf{S E}}\left(\vec{k}_{1}, \vec{k}_{2}, \vec{k}_{3}, \vec{k}_{4}\right)=\frac{1}{\prod_{i=1}^{4} k_{i}^{3}}\left[\left(k_{1}^{3} k_{2}^{3}+k_{3}^{3} k_{4}^{3}\right)\left(k_{13}^{-3}+k_{14}^{-3}\right)\right. \\
+\left(k_{1}^{3} k_{4}^{3}+k_{2}^{3} k_{3}^{3}\right)\left(k_{12}^{-3}+k_{13}^{-3}\right) \\
\left.+\left(k_{1}^{3} k_{3}^{3}+k_{2}^{3} k_{4}^{3}\right)\left(k_{12}^{-3}+k_{14}^{-3}\right)\right] \\
\\
+\left\{\tau_{\mathrm{NL}}^{\mathbf{S E}} P_{\zeta(1)}^{3}+\frac{54}{25} g_{\mathrm{NL}}^{\mathbf{S E}} P_{\zeta(2)}^{3}\right\},
\end{aligned}
$$

where $\tau_{\mathrm{NL}}^{\mathrm{SE}}$ and $g_{\mathrm{NL}}^{\mathrm{SE}}$ are the two nonlinear parameters which carry the signatures of primordial non-Gaussianities of the curvature perturbation obtained from the scalar exchange contribution in the trispectrum analysis. By knowing $\tau_{\mathrm{NL}}^{\mathbf{S E}}$ the other parameter, $g_{\mathrm{NL}}^{\mathrm{SE}}$, can be calculated by making use of the following relation [65]:

$$
\begin{aligned}
& g_{\mathrm{NL}}^{\mathrm{SE}}=\frac{64}{\bar{K}^{3}}\left[\left(k_{1}^{3} k_{2}^{3}+k_{3}^{3} k_{4}^{3}\right)\left(k_{13}^{-3}+k_{14}^{-3}\right)+\left(k_{1}^{3} k_{4}^{3}+k_{2}^{3} k_{3}^{3}\right)\right. \\
& \left.\times\left(k_{12}^{-3}+k_{13}^{-3}\right)+\left(k_{1}^{3} k_{3}^{3}+k_{2}^{3} k_{4}^{3}\right)\left(k_{12}^{-3}+k_{14}^{-3}\right)\right] \tau_{\mathrm{NL}}^{\mathbf{S E}},
\end{aligned}
$$

where $\bar{K}=k_{1}+k_{2}+k_{3}+k_{4}$. The explicit form of $\tau_{\mathrm{NL}}^{\mathbf{S E}}$ characterizing the scalar exchange trispectrum can be expressed for our model as:

$$
\begin{aligned}
& \tau_{\mathrm{NL}}^{\mathrm{SE}}=\frac{2^{8 v_{s}-14} \bar{K}^{10 v_{s}-15} \operatorname{Cos}\left(\left[v_{s}-\frac{1}{2}\right] \frac{\pi}{2}\right)}{\left[\left(k_{1}^{3} k_{2}^{3}+k_{3}^{3} k_{4}^{3}\right)\left(k_{13}^{-3}+k_{14}^{-3}\right)+\left(k_{1}^{3} k_{4}^{3}+k_{2}^{3} k_{3}^{3}\right)\left(k_{12}^{-3}+k_{13}^{-3}\right)+\left(k_{1}^{3} k_{3}^{3}+k_{2}^{3} k_{4}^{3}\right)\left(k_{12}^{-3}+k_{14}^{-3}\right)\right]} \\
& \times\left|\frac{\Gamma\left(v_{s}\right)}{\Gamma\left(\frac{3}{2}\right)}\right|^{8} \frac{\left(1-\epsilon_{V}-s_{V}^{S}\right)^{8} H^{8}}{Y_{S}^{5} c_{S}^{6}\left(k_{1} k_{2} k_{3} k_{4}\right)^{2 v_{s}}}\left\{9 \mathbf{A}^{2}\left[\Xi_{1}\left(-k_{1},-k_{2},-k_{12}, k_{3}, k_{4}, k_{12}\right)-\Xi_{1}\left(k_{1}, k_{2},-k_{12}, k_{3}, k_{4}, k_{12}\right)\right]\right. \\
& +\mathbf{A B}\left[3\left(\vec{k}_{3} \cdot \vec{k}_{4}\right)\left\{\Xi_{3}\left(k_{1}, k_{2},-k_{12}, k_{12}, k_{3}, k_{4}\right)-\Xi_{3}\left(-k_{1},-k_{2},-k_{12}, k_{12}, k_{3}, k_{4}\right)\right\}\right. \\
& +6\left(\overrightarrow{k_{12}} \cdot \vec{k}_{4}\right)\left\{\Xi_{3}\left(k_{1}, k_{2},-k_{12}, k_{3}, k_{4}, k_{12}\right)-\Xi_{3}\left(-k_{1},-k_{2},-k_{12}, k_{3}, k_{4}, k_{12}\right)\right\} \\
& +3\left(\vec{k}_{1} \cdot \vec{k}_{2}\right)\left\{\Xi_{4}\left(-k_{12}, k_{1}, k_{2}, k_{3}, k_{4}, k_{12}\right)-\Xi_{4}\left(-k_{12},-k_{1},-k_{2}, k_{3}, k_{4}, k_{12}\right)\right\} \\
& \left.-6\left(\overrightarrow{k_{12}} \cdot \overrightarrow{k_{2}}\right)\left\{\Xi_{4}\left(k_{1}, k_{2},-k_{12}, k_{3}, k_{4}, k_{12}\right)-\Xi_{4}\left(-k_{1},-k_{2},-k_{12}, k_{3}, k_{4}, k_{12}\right)\right\}\right] \\
& -\mathbf{B}^{2}\left[\left(\vec{k}_{1} \cdot \vec{k}_{2}\right)\left(\vec{k}_{3} \cdot \vec{k}_{4}\right)\left\{\Xi_{2}\left(-k_{12}, k_{1}, k_{2}, k_{12}, k_{3}, k_{4}\right)-\Xi_{2}\left(-k_{12},-k_{1},-k_{2}, k_{12}, k_{3}, k_{4}\right)\right\}\right. \\
& +2\left(\vec{k}_{1} \cdot \vec{k}_{2}\right)\left(\overrightarrow{k_{12}} \cdot \vec{k}_{4}\right)\left\{\Xi_{2}\left(-k_{12}, k_{1}, k_{2}, k_{3}, k_{4}, k_{12}\right)-\Xi_{2}\left(-k_{12},-k_{1},-k_{2}, k_{3}, k_{4}, k_{12}\right)\right\} \\
& -2\left(\overrightarrow{k_{3}} \cdot \overrightarrow{k_{4}}\right)\left(\overrightarrow{k_{12}} \cdot \vec{k}_{2}\right)\left\{\Xi_{2}\left(k_{1}, k_{2},-k_{12}, k_{12}, k_{3}, k_{4}\right)-\Xi_{2}\left(-k_{1},-k_{2},-k_{12}, k_{12}, k_{3}, k_{4}\right)\right\} \\
& \left.-4\left(\overrightarrow{k_{12}} \cdot \overrightarrow{k_{4}}\right)\left(\overrightarrow{k_{12}} \cdot \overrightarrow{k_{2}}\right)\left\{\Xi_{2}\left(k_{1}, k_{2},-k_{12}, k_{3}, k_{4}, k_{12}\right)-\Xi_{2}\left(-k_{1},-k_{2},-k_{12}, k_{3}, k_{4}, k_{12}\right)\right\}\right] \\
& \left.+23 \text { permutations of }\left(k_{1}, k_{2}, k_{3}, k_{4}\right)\right\} \text {, }
\end{aligned}
$$


where the momentum dependent functions $\Xi_{i} \forall i$ are mentioned in the Appendix. Further, using the equilateral configuration the non-Gaussian parameter from the scalar exchange contribution can be expressed as where in the interaction picture the Hamiltonian can be written in terms of the third-order Lagrangian density as

$$
\begin{aligned}
\tau_{\mathrm{NL}}^{\text {equil; SE }}= & \frac{2^{20 v_{s}-42} k^{10 v_{s}-18} \operatorname{Cos}\left(\left[v_{s}-\frac{1}{2}\right] \frac{\pi}{2}\right)}{9 \sqrt{3}}\left|\frac{\Gamma\left(v_{s}\right)}{\Gamma\left(\frac{3}{2}\right)}\right|^{8} \frac{\left(1-\epsilon_{V}-s_{V}^{S}\right)^{8} H^{8}}{Y_{S}^{5} c_{s}^{6}} \\
& +\left\{9 \mathbf{A}^{2}\left[\Xi_{1}\left(-k,-k,-\frac{2 k}{\sqrt{3}}, k, k, \frac{2 k}{\sqrt{3}}\right)-\Xi_{1}\left(k, k,-\frac{2 k}{\sqrt{3}}, k, k, \frac{2 k}{\sqrt{3}}\right)\right]\right. \\
& +k^{2} \mathbf{A B}\left[3\left\{\Xi_{3}\left(k, k,-\frac{2 k}{\sqrt{3}}, \frac{2 k}{\sqrt{3}}, k, k\right)-\Xi_{3}\left(-k,-k,-\frac{2 k}{\sqrt{3}}, \frac{2 k}{\sqrt{3}}, k, k\right)\right\}\right. \\
& +4 \sqrt{3}\left\{\Xi_{3}\left(k, k,-\frac{2 k}{\sqrt{3}}, k, k, \frac{2 k}{\sqrt{3}}\right)-\Xi_{3}\left(-k,-k,-\frac{2 k}{\sqrt{3}}, k, k, \frac{2 k}{\sqrt{3}}\right)\right\} \\
& +3\left\{\Xi_{4}\left(-\frac{2 k}{\sqrt{3}}, k, k, k, k, \frac{2 k}{\sqrt{3}}\right)-\Xi_{4}\left(-\frac{2 k}{\sqrt{3}},-k,-k, k, k, \frac{2 k}{\sqrt{3}}\right)\right\} \\
& \left.-4 \sqrt{3}\left\{\Xi_{4}\left(k, k,-\frac{2 k}{\sqrt{3}}, k, k, \frac{2 k}{\sqrt{3}}\right)-\Xi_{4}\left(-k,-k,-\frac{2 k}{\sqrt{3}}, k, k, \frac{2 k}{\sqrt{3}}\right)\right\}\right] \\
& -k^{4} \mathbf{B}^{2}\left[\left\{\Xi_{2}\left(-\frac{2 k}{\sqrt{3}}, k, k, \frac{2 k}{\sqrt{3}}, k, k\right)-\Xi_{2}\left(-\frac{2 k}{\sqrt{3}},-k,-k, \frac{2 k}{\sqrt{3}}, k, k\right)\right\}\right. \\
& +\frac{4}{\sqrt{3}}\left\{\Xi_{2}\left(-\frac{2 k}{\sqrt{3}}, k, k, k, k, \frac{2 k}{\sqrt{3}}\right)-\Xi_{2}\left(-\frac{2 k}{\sqrt{3}},-k,-k, k, k, \frac{2 k}{\sqrt{3}}\right)\right\} \\
& -\frac{4}{\sqrt{3}}\left\{\Xi_{2}\left(k, k,-\frac{2 k}{\sqrt{3}}, \frac{2 k}{\sqrt{3}}, k, k\right)-\Xi_{2}\left(-k,-k,-\frac{2 k}{\sqrt{3}}, \frac{2 k}{\sqrt{3}}, k, k\right)\right\} \\
& \left.\left.-\frac{16}{3}\left\{\Xi_{2}\left(k, k,-\frac{2 k}{\sqrt{3}}, k, k, \frac{2 k}{\sqrt{3}}\right)-\Xi_{2}\left(-k,-k,-\frac{2 k}{\sqrt{3}}, k, k, \frac{2 k}{\sqrt{3}}\right)\right\}\right]\right\} \\
& +23 \text { permutations }\} .
\end{aligned}
$$

\subsection{Graviton exchange}

In this section we are interested to evaluate the contribution of four-point function of curvature perturbations from the exchange of graviton. This process involves a third-order interaction among scalar fluctuations and tensor perturbations. To proceed, we need here only the significant third order term in the action, which describes the graviton-scalarscalar vertex in the uniform gauge as

$S_{\mathbf{G E}}=\frac{1}{2} \int \mathrm{d} t \mathrm{~d}^{3} x a^{2} \mathcal{Y}_{1} h_{i j} \zeta_{, i} \zeta, j$,

where $\mathcal{Y}_{1}=Y_{S} c_{S}^{2}$. Using the in-in procedure the four-point correlator function for the quasi-exponential limit can be expressed in the following form:

$$
\begin{aligned}
& \left\langle\zeta\left(\overrightarrow{k_{1}}\right) \zeta\left(\vec{k}_{2}\right) \zeta\left(\vec{k}_{3}\right) \zeta\left(\vec{k}_{4}\right)\right\rangle \\
& \quad=-i \lim _{\eta^{\star} \rightarrow 0} \int_{\vec{\star}}^{\eta_{\star}} \mathrm{d} \eta \int_{-\infty}^{\eta} \mathrm{d} \tilde{\eta} \\
& \langle 0|\left[\left[\zeta\left(\vec{k}_{1}\right) \zeta\left(\vec{k}_{2}\right) \zeta\left(\vec{k}_{3}\right) \zeta\left(\vec{k}_{4}\right),\left(H_{\text {int }}(\eta)\right)_{\zeta \zeta \zeta}^{\mathbf{G E}}\right],\right. \\
& \left.\quad \times\left(H_{\text {int }}(\tilde{\eta})\right)_{\zeta \zeta \zeta}^{\mathbf{G E}}\right]|0\rangle \\
& \quad=(2 \pi)^{3} \delta^{(3)}\left(\vec{k}_{1}+\vec{k}_{2}+\vec{k}_{3}+\vec{k}_{4}\right) \mathcal{T}_{\zeta}^{\mathbf{G E}}\left(\vec{k}_{1}, \vec{k}_{2}, \vec{k}_{3}, \vec{k}_{4}\right),
\end{aligned}
$$

Here following the ansatz used in [22] the trispectrum $\mathcal{T}_{\zeta}^{\mathbf{G E}}\left(\vec{k}_{1}, \vec{k}_{2}, \vec{k}_{3}, \vec{k}_{4}\right)$ obtained from the graviton exchange contribution is defined as

$$
\begin{aligned}
& \mathcal{T}_{\zeta}^{\mathbf{G E}}\left(\vec{k}_{1}, \vec{k}_{2}, \vec{k}_{3}, \vec{k}_{4}\right)=\frac{1}{\prod_{i=1}^{4} k_{i}^{3}}\left[\left(k_{1}^{3} k_{2}^{3}+k_{3}^{3} k_{4}^{3}\right)\left(k_{13}^{-3}+k_{14}^{-3}\right)\right. \\
& +\left(k_{1}^{3} k_{4}^{3}+k_{2}^{3} k_{3}^{3}\right)\left(k_{12}^{-3}+k_{13}^{-3}\right) \\
& \left.+\left(k_{1}^{3} k_{3}^{3}+k_{2}^{3} k_{4}^{3}\right)\left(k_{12}^{-3}+k_{14}^{-3}\right)\right]\left\{\tau_{\mathrm{NL}}^{\mathbf{G E}} P_{\zeta(1)}^{3}\right. \\
& \left.\quad+\frac{54}{25} g_{\mathrm{NL}}^{\mathbf{G E}} P_{\zeta(2)}^{3}\right\},
\end{aligned}
$$

where $\tau_{\mathrm{NL}}^{\mathbf{G E}}$ and $g_{\mathrm{NL}}^{\mathbf{G E}}$ are the two nonlinear parameters which carry the signatures of primordial non-Gaussianities of the curvature perturbation in the trispectrum analysis. By knowing $\tau_{\mathrm{NL}}^{\mathbf{G E}}$ the other parameter $g_{\mathrm{NL}}^{\mathbf{G E}}$ can be calculated by making use of the following relation [65]:

$$
\begin{aligned}
& g_{\mathrm{NL}}^{\mathbf{G E}}=\frac{64}{\bar{K}^{3}}\left[\left(k_{1}^{3} k_{2}^{3}+k_{3}^{3} k_{4}^{3}\right)\left(k_{13}^{-3}+k_{14}^{-3}\right)+\left(k_{1}^{3} k_{4}^{3}+k_{2}^{3} k_{3}^{3}\right)\right. \\
& \left.\times\left(k_{12}^{-3}+k_{13}^{-3}\right)+\left(k_{1}^{3} k_{3}^{3}+k_{2}^{3} k_{4}^{3}\right)\left(k_{12}^{-3}+k_{14}^{-3}\right)\right] \tau_{\mathrm{NL}}^{\mathbf{G E}},
\end{aligned}
$$


where $\bar{K}=k_{1}+k_{2}+k_{3}+k_{4}$. The explicit form of $\tau_{\mathrm{NL}}^{\mathbf{G E}}$ characterizing the trispectrum obtained from the graviton exchange contribution can be expressed for our model as: tor as $\vec{k}_{a}=k_{a}\left(\sin \theta_{a} \cos \phi_{a}, \sin \theta_{a} \sin \phi_{a}, \cos \theta_{a}\right)$, where $\cos \theta_{a} \equiv \overrightarrow{\hat{k}}_{a} \cdot \overrightarrow{\hat{k}}_{12}$ and $\cos \phi_{a} \equiv \overrightarrow{\hat{k}}_{a} \cdot \vec{e}$. This implies

$$
\begin{aligned}
\tau_{\mathrm{NL}}^{\mathbf{G E}}= & \lim _{\eta^{\star} \rightarrow 0} \frac{2^{8 v_{s}-31} \pi^{2}\left(1-\epsilon_{V}-s_{V}^{S}\right)^{8}\left(1-\epsilon_{V}-s_{V}^{T}\right)^{2} \bar{K}^{10 v_{s}-15} \operatorname{Sin}^{8}\left(\left[v_{s}-\frac{1}{2}\right] \frac{\pi}{2}\right) \operatorname{Sin}^{2}\left(\left[v_{T}-\frac{1}{2}\right] \frac{\pi}{2}\right)}{\left[\left(k_{1}^{3} k_{2}^{3}+k_{3}^{3} k_{4}^{3}\right)\left(k_{13}^{-3}+k_{14}^{-3}\right)+\left(k_{1}^{3} k_{4}^{3}+k_{2}^{3} k_{3}^{3}\right)\left(k_{12}^{-3}+k_{13}^{-3}\right)+\left(k_{1}^{3} k_{3}^{3}+k_{2}^{3} k_{4}^{3}\right)\left(k_{12}^{-3}+k_{14}^{-3}\right)\right]} \\
& \times\left|\frac{\Gamma\left(v_{s}\right)}{\Gamma\left(\frac{3}{2}\right)}\right|^{8}\left|\frac{\Gamma\left(v_{T}\right)}{\Gamma\left(\frac{3}{2}\right)}\right|^{2} \frac{H^{10}}{Y_{S}^{4} Y_{T}^{2} c_{s}^{12} c_{T}^{6}}\left\{\sum_{\substack{\lambda=+[+,], \times[+,-]}} \sum_{\substack { i, j, l, m \\
\begin{subarray}{c}{a<b \\
c<d \\
23{ i , j , l , m \\
\begin{subarray} { c } { a < b \\
c < d \\
2 3 } }\end{subarray}} \epsilon_{i j}^{\lambda}\left(\vec{k}_{a b}\right) \epsilon_{l m}^{\lambda}\left(\vec{k}_{c d}\right) \frac{k_{a}^{i} k_{b}^{j} k_{c}^{l} k_{d}^{m}}{k_{a b}^{v_{T}+3}\left(k_{a} k_{b} k_{c} k_{d}\right)^{2 v_{s}}} \cdot \vartheta_{a b c d}\left(\eta_{\star}\right)\right\} .
\end{aligned}
$$

The momentum dependent functions $\vartheta_{a b c d}\left(\eta_{\star}\right)$ are given in the Appendix. Here to write Eq. (4.24) we have used the fact that the exchange momentum dependent polarization tensor $\epsilon_{i j}^{\lambda}\left(\vec{k}_{a b}\right)$ is a symmetric tensor and also the four-point correlator obtained from the graviton exchange is invariant under the exchange of the subscripts of the momenta, $a \leftrightarrow b$ and $c \leftrightarrow d$. Additionally in Eq. (4.24) the sum is performed only over different indices $a, b, c, d$, and we have extracted an overall symmetry factor of 4 , which takes care of the exchanges $a \leftrightarrow b$ and $c \leftrightarrow d$. Rewriting the sums appearing in Eq. (4.24) we get the following reduced formula for the non-Gaussian parameter:

$$
\begin{gathered}
\epsilon_{i j}^{+} k_{1}^{i} k_{2}^{j}=k_{1} k_{2} \sin \theta_{1} \sin \theta_{2} \cos \left(\phi_{1}+\phi_{2}\right), \\
\epsilon_{i j}^{\times} k_{1}^{i} k_{2}^{j}=k_{1} k_{2} \sin \theta_{1} \sin \theta_{2} \sin \left(\phi_{1}+\phi_{2}\right),
\end{gathered}
$$

with an identical relation holding for $\epsilon_{i j}^{+} k_{3}^{i} k_{4}^{j}$ and $\epsilon_{i j}^{\times} k_{3}^{i} k_{4}^{j}$, which will contribute to the polarization sum also. The projections of the momentum vectors $\vec{k}_{1}$ and $\vec{k}_{2}$ (similarly for $\vec{k}_{3}$ and $\vec{k}_{4}$ ) on the plane orthogonal to the exchange momentum vector $\vec{k}_{12}\left(\vec{k}_{34}\right)$ have the same amplitude but opposite directions. Consequently we have two additional sets of constraint relationships given by

$$
\begin{aligned}
& \tau_{\mathrm{NL}}^{\mathbf{G E}}=\frac{2^{8 v_{s}-31} \pi^{2}\left(1-\epsilon_{V}-s_{V}^{S}\right)^{8}\left(1-\epsilon_{V}-s_{V}^{T}\right)^{2} \bar{K}^{10 v_{s}-15} \operatorname{Sin}^{8}\left(\left[v_{s}-\frac{1}{2}\right] \frac{\pi}{2}\right) \operatorname{Sin}^{2}\left(\left[v_{T}-\frac{1}{2}\right] \frac{\pi}{2}\right)}{\left[\left(k_{1}^{3} k_{2}^{3}+k_{3}^{3} k_{4}^{3}\right)\left(k_{13}^{-3}+k_{14}^{-3}\right)+\left(k_{1}^{3} k_{4}^{3}+k_{2}^{3} k_{3}^{3}\right)\left(k_{12}^{-3}+k_{13}^{-3}\right)+\left(k_{1}^{3} k_{3}^{3}+k_{2}^{3} k_{4}^{3}\right)\left(k_{12}^{-3}+k_{14}^{-3}\right)\right]} \\
& \times\left|\frac{\Gamma\left(v_{s}\right)}{\Gamma\left(\frac{3}{2}\right)}\right|^{8}\left|\frac{\Gamma\left(v_{T}\right)}{\Gamma\left(\frac{3}{2}\right)}\right|^{2} \frac{H^{10}}{Y_{S}^{4} Y_{T}^{2} c_{s}^{12} c_{T}^{6}} \sum_{\substack{\lambda=+[+,-], \times[+,-]}} \sum_{i, j, l, m}\left[\epsilon_{i j}^{\lambda}\left(\vec{k}_{12}\right) \epsilon_{l m}^{\lambda}\left(\vec{k}_{34}\right) \frac{k_{1}^{i} k_{2}^{j} k_{3}^{l} k_{4}^{m}}{k_{12}^{\nu_{T}+3}\left(k_{1} k_{2} k_{3} k_{4}\right)^{2 v_{s}}} \cdot\left(\hat{\vartheta}_{1234}+\hat{\vartheta}_{3412}\right)\right. \\
& \left.\left.+\epsilon_{i j}^{\lambda}\left(\vec{k}_{13}\right) \epsilon_{l m}^{\lambda}\left(\vec{k}_{24}\right) \frac{k_{1}^{i} k_{3}^{j} k_{2}^{l} k_{4}^{m}}{k_{13}^{\nu_{T}+3}\left(k_{1} k_{3} k_{2} k_{4}\right)^{2 v_{s}}} \cdot\left(\hat{\vartheta}_{1324}+\hat{\vartheta}_{2413}\right)+\epsilon_{i j}^{\lambda}\left(\vec{k}_{14}\right) \epsilon_{l m}^{\lambda}\left(\vec{k}_{23}\right) \frac{k_{1}^{i} k_{4}^{j} k_{2}^{l} k_{3}^{m}}{k_{14}^{v_{T}+3}\left(k_{1} k_{4} k_{2} k_{3}\right)^{2 v_{s}}} \cdot\left(\hat{\vartheta}_{1423}+\hat{\vartheta}_{2314}\right)\right]\right\},
\end{aligned}
$$

where we define $\lim _{\eta_{\star} \rightarrow 0} \vartheta_{a b c d}\left(\eta_{\star}\right):=\hat{\vartheta}_{a b c d}$. There are divergent contributions in the limit $\eta_{*} \rightarrow 0$ that appear with a logarithmic dependence on the momenta, but the additive cumulative contributions of $\mathcal{I}_{a b c d}$ and $\mathcal{I}_{c d a b}$ give rise to a finite contribution at late times.

To represent Eq. (4.25) in a simpler form, let us start with the polarization sum $\sum_{s} \epsilon_{i j}^{s}\left(\vec{k}_{12}\right) \epsilon_{l m}^{s}\left(\vec{k}_{34}\right) k_{1}^{i} k_{2}^{j} k_{3}^{l} k_{4}^{m}$ in terms of the relative angles between the $\vec{k}_{a}$ and $\vec{k}_{12}$. The polarization tensors $\epsilon_{i j}^{s}$ can be rewritten as

$$
\epsilon_{i j}^{+}=\vec{e}_{i} \otimes \vec{e}_{j}-\overrightarrow{\bar{e}}_{i} \otimes \overrightarrow{\bar{e}}_{j}, \quad \epsilon_{i j}^{\times}=\vec{e}_{i} \otimes \overrightarrow{\bar{e}}_{j}+\overrightarrow{\bar{e}}_{i} \otimes \vec{e}_{j},
$$

where $\vec{e}$ and $\overrightarrow{\bar{e}}$ are orthogonal unit vectors perpendicular to exchange momentum vector $\vec{k}_{12}$. It is convenient to write the momentum vector $\vec{k}_{a}$ in a spherical polar coordinate system having $\left\{\vec{e}, \overrightarrow{\bar{e}}, \overrightarrow{\hat{k}}_{12} \equiv \vec{k}_{12} / k_{12}\right\}$ as a basis. In this coordinate system one can express the momentum vec- $k_{2} \sin \theta_{2}=k_{1} \sin \theta_{1}$ and $\phi_{2}=\phi_{1}+\pi$,

$$
k_{4} \sin \theta_{4}=k_{3} \sin \theta_{3} \text { and } \phi_{4}=\phi_{3}+\pi \text {. }
$$

Using these relations we get

$$
\begin{aligned}
& \sum_{s} \epsilon_{i j}^{s}\left(\vec{k}_{a b}\right) \epsilon_{l m}^{s}\left(\vec{k}_{c d}\right) k_{a}^{i} k_{b}^{j} k_{c}^{l} k_{d}^{m} \\
& =k_{a}^{2} k_{c}^{2} \sin ^{2} \theta_{a} \sin ^{2} \theta_{c} \cos 2 \Upsilon_{a b, c d},
\end{aligned}
$$

where we define a new angular coordinate $\Upsilon_{a b, c d} \equiv \phi_{a}-\phi_{c}$ with $a=1,(b, c)=2,3,4, d=3,4$, and $b>a, d>$ $c, a \neq b \neq c \neq d$, which physically represents the angle between the projections of the two momentum vectors $\vec{k}_{a}$ and $\vec{k}_{c}$ on the plane orthogonal to $\vec{k}_{12}$. Alternatively this can be interpreted as the angle between the two planes formed by the pair of momentum vectors $\left\{\vec{k}_{1}, \vec{k}_{2}\right\}$ and $\left\{\vec{k}_{3}, \vec{k}_{4}\right\}$. Thus, the expression for the non-Gaussian parameter calculated from the graviton exchange contribution from the trispectrum can be simplified to the following expression: 


$$
\begin{aligned}
\tau_{\mathrm{NL}}^{\mathbf{G E}}= & \frac{2^{8 v_{s}-31} \pi^{2}\left(1-\epsilon_{V}-s_{V}^{S}\right)^{8}\left(1-\epsilon_{V}-s_{V}^{T}\right)^{2} \bar{K}^{10 v_{s}-15} \operatorname{Sin}^{8}\left(\left[v_{s}-\frac{1}{2}\right] \frac{\pi}{2}\right) \operatorname{Sin}^{2}\left(\left[v_{T}-\frac{1}{2}\right] \frac{\pi}{2}\right)}{\left[\left(k_{1}^{3} k_{2}^{3}+k_{3}^{3} k_{4}^{3}\right)\left(k_{13}^{-3}+k_{14}^{-3}\right)+\left(k_{1}^{3} k_{4}^{3}+k_{2}^{3} k_{3}^{3}\right)\left(k_{12}^{-3}+k_{13}^{-3}\right)+\left(k_{1}^{3} k_{3}^{3}+k_{2}^{3} k_{4}^{3}\right)\left(k_{12}^{-3}+k_{14}^{-3}\right)\right]} \\
& \times\left|\frac{\Gamma\left(v_{s}\right)}{\Gamma\left(\frac{3}{2}\right)}\right|^{8}\left|\frac{\Gamma\left(v_{T}\right)}{\Gamma\left(\frac{3}{2}\right)}\right|^{2} \frac{H^{10}}{Y_{S}^{4} Y_{T}^{2} c_{s}^{12} c_{T}^{6}}\left\{\frac{k_{1}^{2} k_{3}^{2}\left[1-\left(\hat{\vec{k}}_{1} \cdot \hat{\vec{k}}_{12}\right)^{2}\right]\left[1-\left(\hat{\vec{k}}_{3} \cdot \hat{\vec{k}}_{12}\right)^{2}\right]}{k_{12}^{\nu_{T}+3}\left(k_{1} k_{2} k_{3} k_{4}\right)^{2 v_{s}}} \cos 2 \Upsilon_{12,34} \cdot\left(\hat{\vartheta}_{1234}+\hat{\vartheta}_{3412}\right)\right. \\
& +\frac{k_{1}^{2} k_{2}^{2}\left[1-\left(\hat{\vec{k}}_{1} \cdot \hat{\vec{k}}_{13}\right)^{2}\right]\left[1-\left(\hat{\vec{k}}_{2} \cdot \hat{\vec{k}}_{13}\right)^{2}\right]}{k_{13}^{\nu_{T}+3}\left(k_{1} k_{3} k_{2} k_{4}\right)^{2 v_{s}} \cos 2 \Upsilon_{13,24} \cdot\left(\hat{\vartheta}_{1324}+\hat{\vartheta}_{2413}\right)} \\
& \left.+\frac{k_{1}^{2} k_{2}^{2}\left[1-\left(\hat{\vec{k}}_{1} \cdot \hat{\vec{k}}_{14}\right)^{2}\right]\left[1-\left(\hat{\vec{k}}_{2} \cdot \hat{\vec{k}}_{14}\right)^{2}\right]}{k_{14}^{\nu_{T}+3}\left(k_{1} k_{4} k_{2} k_{3}\right)^{2 v_{s}}} \cos 2 \Upsilon_{14,23} \cdot\left(\hat{\vartheta}_{1423}+\hat{\vartheta}_{2314}\right)\right\} .
\end{aligned}
$$

Further, incorporating the contribution from the maximum shape of the trispectrum one can show that the graviton exchange contribution does not contribute anything in the equilateral limit. Now summing up all the significant contributions of the four-point four-scalar correlation coming from the contact interaction, scalar exchange, and graviton exchange interaction the numerical value of $\tau_{\mathrm{NL}}^{\text {equil }}$ in the equilateral limit is obtained from our setup as $48<\tau_{\mathrm{NL}}^{\text {equil }}<97$ in the quasi-exponential limit within the window for tensor-toscalar ratio $0.213<r<0.250$ which is significantly large from other class of DBI models and consistent with the combined constraint obtained from the Planck +WMAP9+high$L+B I C E P 2[2-5]$ data.

\section{Four-point consistency conditions and violation of Suyama-Yamaguchi relation}

In the counter-collinear limit collecting the contribution from the scalar exchange diagram we derive the following expression for the four-point consistency condition:

$$
\begin{aligned}
& \left\langle\zeta\left(\vec{k}_{1}\right) \zeta\left(\vec{k}_{2}\right) \zeta\left(\vec{k}_{3}\right) \zeta\left(\vec{k}_{4}\right)\right\rangle^{\mathrm{SE}} \approx(2 \pi)^{3} \delta^{3}\left(\vec{k}_{1}+\vec{k}_{2}+\vec{k}_{3}+\vec{k}_{4}\right) \\
& \quad \times \frac{\left(n_{\zeta}-1\right)^{2}}{4} P_{\zeta}\left(k_{12}\right) P_{\zeta}\left(k_{1}\right)\left[P_{\zeta}\left(k_{3}\right)+\cdots\right]
\end{aligned}
$$

which can be interpreted as the scalar exchange contribution arising from the product of two back-to-back bispectra in the squeezed limit. Additionally, we consider the contribution from the graviton exchange diagram from which we derive another expression for the four-point consistency condition:

$$
\begin{aligned}
& \left\langle\zeta\left(\vec{k}_{1}\right) \zeta\left(\vec{k}_{2}\right) \zeta\left(\vec{k}_{3}\right) \zeta\left(\vec{k}_{4}\right)\right\rangle^{G E} \approx 9 c_{s} \epsilon_{s}(2 \pi)^{3} \delta^{3} \\
& \quad\left(\vec{k}_{1}+\vec{k}_{2}+\vec{k}_{3}+\vec{k}_{4}\right) P_{\zeta}\left(k_{12}\right) P_{\zeta}\left(k_{1}\right) \\
& \quad \times\left[\sum_{\substack{\lambda=+[+,-] \\
\times[+,-]}} \sum_{i, j, l, m} \epsilon_{i j}^{\lambda}\left(\vec{k}_{12}\right) \epsilon_{l m}^{\lambda}\left(\vec{k}_{34}\right) \frac{k_{1}^{i} k_{1}^{j} k_{3}^{l} k_{3}^{m}}{k_{1}^{2} k_{3}^{2}} P_{\zeta}\left(k_{3}\right)+\cdots\right] .
\end{aligned}
$$

Here using $k_{12} \rightarrow 0, \theta_{1}, \theta_{3} \rightarrow \pi$ the polarization sum appearing in Eq. (5.2) can be simplified to the following expression:

$$
\sum_{\substack{\lambda=+[+,-], \times[+,-]}} \sum_{i, j, l, m} \epsilon_{i j}^{\lambda}\left(\vec{k}_{12}\right) \epsilon_{l m}^{\lambda}\left(\vec{k}_{34}\right) \frac{k_{1}^{i} k_{1}^{j} k_{3}^{l} k_{3}^{m}}{k_{1}^{2} k_{3}^{2}}=\cos 2 \Upsilon_{12,34} .
$$

Further substituting Eq. (5.3) in Eq. (5.2) and using Eq. (3.15) the four-point correlation function from the graviton exchange contribution in the counter-collinear limit $\left(k_{12}<<k_{1} \approx\right.$ $k_{2}, k_{3} \approx k_{4}$ ) reduces to the following expression:

$$
\begin{aligned}
& \left\langle\zeta\left(\vec{k}_{1}\right) \zeta\left(\vec{k}_{2}\right) \zeta\left(\vec{k}_{3}\right) \zeta\left(\vec{k}_{4}\right)\right\rangle^{\mathrm{GE}}=9.2^{2\left(v_{s}-v_{T}-4\right)} r_{\star} . \\
& \quad\left[1+\frac{3}{2} \mathcal{O}\left(\epsilon_{T}^{2}\right)\right]_{\star}\left(\frac{1-\epsilon_{V}-s_{V}^{S}}{1-\epsilon_{V}-s_{V}^{T}}\right)_{\star}^{2} \cdot\left|\frac{\Gamma\left(v_{s}\right)}{\Gamma\left(v_{T}\right)}\right|^{2} \\
& \quad \times(2 \pi)^{3} \delta^{3}\left(\vec{k}_{1}+\vec{k}_{2}+\vec{k}_{3}+\vec{k}_{4}\right) P_{\zeta}\left(k_{12}\right) P_{\zeta}\left(k_{1}\right) \\
& \quad \times\left[\cos 2 \Upsilon_{12,34} P_{\zeta}\left(k_{3}\right)+\cdots\right] .
\end{aligned}
$$

To check the validity of the well-known Suyama-Yamguchi consistency relation we start with the in-in picture where the four-point correlator can be written as

$$
\left\langle\zeta^{2}(\vec{x}) \zeta^{2}(0)\right\rangle_{\vec{k}}=\sum_{n}\left|\left\langle n_{\vec{k}} \mid \zeta^{2}(0)\right\rangle\right|^{2}
$$

where $n$ is a label for the individual states or particle number within the momentum eigenspace. Here the sum is written over positive definite terms. On the other hand in this context one of the contributions is the square of the squeezed limit of the three-point correlation function of the scalar contribution. This implies

$$
\left\langle\zeta^{2}(\vec{x}) \zeta^{2}(0)\right\rangle_{\vec{k}}=\frac{\left|\left\langle\zeta(\vec{k}) \mid \zeta^{2}(0)\right\rangle\right|^{2}}{P_{\zeta}(k)}+\sum_{\tilde{n}}\left|\left\langle\tilde{n}_{\vec{k}} \mid \zeta^{2}(0)\right\rangle\right|^{2} .
$$


As the second term in Eq. (5.6) is always positive definite we conclude that $\left\langle\zeta^{2}(\vec{x}) \zeta^{2}(0)\right\rangle_{\vec{k}} \geq \frac{\left|\left\langle\zeta(\vec{k}) \mid \zeta^{2}(0)\right\rangle\right|^{2}}{P_{\zeta}(k)}$. Further using this result in the quasi-exponential limit we get

$$
\begin{aligned}
& \lim _{q \rightarrow 0} \int_{\overrightarrow{k_{1}}} \frac{\mathrm{d}^{3} k_{1}}{(2 \pi)^{3}} \int_{\overrightarrow{k_{3}}} \frac{\mathrm{d}^{3} k_{3}}{(2 \pi)^{3}}\left\langle\zeta\left(\vec{k}_{1}\right) \zeta(\underbrace{\vec{q}-\overrightarrow{k_{1}}}_{\overrightarrow{k_{2}}}) \zeta\left(\overrightarrow{k_{3}}\right) \zeta(\underbrace{-\vec{q}-\overrightarrow{k_{3}}}_{\overrightarrow{k_{4}}})\right\rangle \\
& \geq \lim _{q \rightarrow 0} \frac{\mid \int_{\overrightarrow{k_{2}}} \frac{\mathrm{d}^{3} k_{2}}{(2 \pi)^{3}}\left\langle\zeta(\underbrace{\vec{k}}_{\overrightarrow{k_{1}}}) \zeta\left(\overrightarrow{k_{2}}\right) \zeta(\left.\underbrace{\left.-\vec{q}-\overrightarrow{k_{2}}\right)}_{\overrightarrow{k_{3}}}\right|^{2}\right.}{P_{\zeta}(q)} .
\end{aligned}
$$

Hence using Eq. (5.7) finally we get

$$
\begin{aligned}
& \lim _{q \rightarrow 0} \int_{\overrightarrow{k_{1}}} \frac{\mathrm{d}^{3} k_{1}}{(2 \pi)^{3}} \int_{\overrightarrow{k_{3}}} \frac{\mathrm{d}^{3} k_{3}}{(2 \pi)^{3}} P_{\zeta}\left(k_{1}\right) P_{\zeta}\left(k_{2}\right)\left\{\tau_{\mathrm{NL}}-\frac{36}{25}\left(f_{\mathrm{NL}}\right)^{2}\right\} \\
& \quad \geq 0,
\end{aligned}
$$

resulting in a generic outcome of the DBI Galileon inflation, viz.,

$\hat{\tau}_{\mathrm{NL}} \geq \frac{36}{25}\left(\hat{f}_{\mathrm{NL}}\right)^{2}$

where $\hat{\tau}_{\mathrm{NL}}$ and $\hat{f}_{\mathrm{NL}}$ are used to represent the soft limits of the three- and four-point correlator functions. This relation directly confirms the partial violation of the standard Suyama-Yamaguchi relation $[61,62,64] \hat{\tau}_{\mathrm{NL}}=\frac{36}{25}\left(\hat{f}_{\mathrm{NL}}\right)^{2}$. These nontrivial features allow us to go beyond the no-go theorem in the present context. Some other aspects of the violation of the well-known consistency relations in the context of single field inflation have been studied in $[65,66]$.

Let us now investigate some possible explanations of the partial violation of the standard Suyama-Yamaguchi relation. The standard relations and limits of Non-Gaussianity are usually derived under the following assumptions:

- The background is Einsteinian gravity.

- Inflation is driven by a single scalar field.

- The scalar field action is canonical.

- Perfect slow-roll conditions hold good throughout.

- The vacuum is Bunch-Davies.

Of course, most of the results derived using these assumptions are true to a great extent; it is not obvious that they will still hold good if one or more assumptions are relaxed. Only when one deals with a context where one has to relax one or more assumptions, one can investigate the consequences and conclude if the relations are still valid or not. In the present scenario, a non-Einstein framework forms the background along with a non-canonical action appearing in the matter sector for a DBI Galileon. The contributions of them arise through the first two terms of Eq. (2.4) which will further effect Eqs. (5.1) and (5.4). On top of that, we have higher derivative contributions for the DBI Galileon matter sector, for the contact interaction, and scalar and graviton exchange contributions are coupled with the higher curvature contributions through highly nonlinear terms as appearing in the perturbative action as mentioned in Eqs. (4.2), (4.13), and (4.20), which directly affects the interaction vertex factors as well as the propagators of the setup, resulting in a deviation from the standard results. We suspect that these non-standard inputs might have reflected in the violation of the no-go theorem.

Having said this, we do admit that this can at best serve as a qualitative explanation of the violation. A huge amount of work needs to be done before one can comment conclusively on the deviation from which the assumption still respects the relation and the deviation which leads to violation, and, in case it does, to what extent. This is a highly nontrivial task which one can only hope to attempt in the future.

\section{Summary and outlook}

In this article we have explored primordial non-Gaussian features of the DBI Galileon inflation in the D3 brane. We have derived the expressions for three- and four-point correlator functions in terms of the nonlinear parameters $f_{\mathrm{NL}}$ and $\tau_{\mathrm{NL}}$ for the equilateral type of non-Gaussian configurations in the nontrivial polarization modes. This resulted in a significantly large value for the non-Gaussianity from this setup. We could also find a parameter space for both non-Gaussianity and the tensor-to-scalar ratio $(r)$ consistent with the combined constraint obtained from the Planck $+W M A P 9+h i g h-L+B I C E P 2$ data. The detectable features of primordial non-Gaussianity lead to the conclusion that this type of models can directly be verified by upcoming data. Moreover, the calculations reveal some other interesting results like a partial violation of the Suyama-Yamaguchi four-point consistency relation.

Some issues which can be addressed in the context of nonGaussianity for the DBI Galileon are studies of mass spectrum of primordial black hole formation $[67,68]$ as a tool for constraining non-Gaussianity at small scales; the effect of the presence of one loop and two loop radiative corrections in the presence of all possible scalar and tensor mode fluctuations in the bispectrum and trispectrum; the study of different shapes in equilateral, local, orthogonal, squeezed limit configuration for the tree, one and two loop level of nonGaussianity and calculation of other higher-order $n$-point correlation functions to find the proper consistency relations between all higher-order non-Gaussian parameters as well as the analysis of CMB bispectrum and trispectrum in the presence of a Galileon in a SUGRA background. Given the promising results of the present paper, these open issues are worth exploring in the future as they may give rise to interesting results. 
Acknowledgments SC thanks the Council of Scientific and Industrial Research, India, for financial support through the Senior Research Fellowship (Grant No. 09/093(0132)/2010).

Open Access This article is distributed under the terms of the Creative Commons Attribution 4.0 International License (http://creativecomm ons.org/licenses/by/4.0/), which permits unrestricted use, distribution, and reproduction in any medium, provided you give appropriate credit to the original author(s) and the source, provide a link to the Creative Commons license, and indicate if changes were made.

Funded by SCOAP ${ }^{3}$.

\section{Appendix}

In this section we mention all the momentum dependent functions appearing in the context of the bispectrum and trispectrum analysis coming from all scalar-tensor three-point correlators and the four-point scalar correlation.

\section{A. Functions appearing in three-scalar correlation}

The functions appearing in the context of the three-scalar correlation can be expressed as
In the equilateral configuration these functions are related through the following expression:

$$
\begin{gathered}
\mathcal{I}_{1}^{\text {equil }}(x)=\mathcal{I}_{5}^{\text {equil }}(x)=\frac{\mathcal{I}_{4}^{\text {equil }}(x)}{2}, \mathcal{I}_{2}^{\text {equil }}(x)=\frac{3 \mathcal{I}_{8}^{\text {equil }}(x)}{2(1-x)} \\
\mathcal{I}_{6}^{\text {equil }}(x)=\left(1+\frac{x}{2}\right) \mathcal{I}_{3}^{\text {equil }}(x) .
\end{gathered}
$$

Additionally in the squeezed limit these functions are reduced to the following expressions:

$$
\begin{aligned}
\mathcal{I}_{1}(x)= & \operatorname{Cos}\left(\left[x-\frac{1}{2}\right] \frac{\pi}{2}\right) \Gamma(1+x)\left[\frac{2+x}{K} \sum_{i>j} k_{i}^{2} k_{j}^{2}-\frac{1+x}{K^{2}} \sum_{i \neq j} k_{i}^{2} k_{j}^{3}\right], \\
\mathcal{I}_{2}(x)= & \operatorname{Cos}\left(\left[x-\frac{1}{2}\right] \frac{\pi}{2}\right) \Gamma(1+x)\left[\frac{K}{1-x}-\frac{1}{K} \sum_{i>j} k_{i} k_{j}-\frac{1+x}{K^{2}} k_{1} k_{2} k_{3}\right], \mathcal{I}_{3}(x)=\frac{\left(k_{1} k_{2} k_{3}\right)^{3}}{K^{3}} \frac{\Gamma(3+x)}{2} \operatorname{Cos}\left(\left[x-\frac{1}{2}\right] \frac{\pi}{2}\right), \\
\mathcal{I}_{4}(x)= & \operatorname{Cos}\left(\left[x-\frac{1}{2}\right] \frac{\pi}{2}\right)\left\{\frac{\left(\overrightarrow{k_{1}} \cdot \overrightarrow{k_{2}}\right) k_{3}^{2}}{K}\left[(3+x) \Gamma(1+x)-\Gamma(2+x) \frac{k_{3}}{K}\right]+\frac{\left(\overrightarrow{k_{2}} \cdot \vec{k}_{3}\right) k_{1}^{2}}{K}\left[(3+x) \Gamma(1+x)-\Gamma(2+x) \frac{k_{1}}{K}\right]\right. \\
& \left.+\frac{\left(\overrightarrow{k_{3}} \cdot \overrightarrow{k_{1}}\right) k_{2}^{2}}{K}\left[(3+x) \Gamma(1+x)-\Gamma(2+x) \frac{k_{2}}{K}\right]\right\}, \\
\mathcal{I}_{5}(x)= & \operatorname{Cos}\left(\left[x-\frac{1}{2}\right] \frac{\pi}{2}\right)\left\{\frac{\left(\overrightarrow{k_{1}} \cdot \overrightarrow{k_{2}}\right) k_{3}^{2}}{K}\left[\Gamma(1+x)+\Gamma(2+x) \frac{k_{3}}{K}\right]+\frac{\left(\overrightarrow{k_{2}} \cdot \overrightarrow{k_{3}}\right) k_{1}^{2}}{K}\left[\Gamma(1+x)+\Gamma(2+x) \frac{k_{1}}{K}\right]\right. \\
& \left.+\frac{\left(\overrightarrow{k_{3}} \cdot \overrightarrow{k_{1}}\right) k_{2}^{2}}{K}\left[\Gamma(1+x)+\Gamma(2+x) \frac{k_{2}}{K}\right]\right\}, \\
\mathcal{I}_{6}(x)= & \frac{\left(k_{1} k_{2} k_{3}\right)^{2}}{K^{3}} \operatorname{Cos}\left(\left[x-\frac{1}{2}\right] \frac{\pi}{2}\right) \frac{(6+x) \Gamma(3+x)}{12}, \\
\mathcal{I}_{7}(x)= & \operatorname{Cos}\left(\left[x-\frac{1}{2}\right] \frac{\pi}{2}\right) \frac{2+x}{2}\left[\Gamma(1+x)+\Gamma(2+x)\left(\frac{k_{1} k_{2}+k_{2} k_{3}+k_{3} k_{1}}{K^{2}}+(3+x) \frac{k_{1} k_{2} k_{3}}{K^{3}}\right)\right]\left\{\frac{\left(\overrightarrow{k_{1}} \cdot \overrightarrow{k_{2}}\right) k_{3}^{2}}{K}+\frac{\left(\overrightarrow{k_{2}} \cdot \overrightarrow{k_{3}}\right) k_{1}^{2}}{K}+\frac{\left(\overrightarrow{k_{3}} \cdot \overrightarrow{k_{1}}\right) k_{1}^{2}}{K}\right\}, \\
\mathcal{I}_{8}(x)= & \operatorname{Cos}\left(\left[x-\frac{1}{2}\right] \frac{\pi}{2}\right)\left\{\frac{\left(\overrightarrow{k_{1}} \cdot \overrightarrow{k_{2}}\right) k_{3}^{2}}{K}\left[(3+x) \Gamma(1+x)+(3+x) \Gamma(2+x) \frac{k_{3}}{K}-\Gamma(3+x) \frac{k_{3}^{2}}{K^{2}}\right]+\frac{\left(\overrightarrow{k_{2}} \cdot \vec{k}_{3}\right) k_{1}^{2}}{K}[(3+x) \Gamma(1+x)\right. \\
& \left.\left.+(3+x) \Gamma(2+x) \frac{k_{1}}{K}-\Gamma(3+x) \frac{k_{1}^{2}}{K^{2}}\right]+\frac{\left(\overrightarrow{k_{3}} \cdot \vec{k}_{1}\right) k_{2}^{2}}{K}\left[(3+x) \Gamma(1+x)+(3+x) \Gamma(2+x) \frac{k_{1}}{K}-\Gamma(3+x) \frac{k_{1}^{2}}{K^{2}}\right]\right\} .
\end{aligned}
$$




$$
\begin{aligned}
& \mathcal{I}_{1}^{\mathrm{sq}}= \operatorname{Cos}\left(\left[x-\frac{1}{2}\right] \frac{\pi}{2}\right) k_{1}^{3} \Gamma(1+x)\left[\frac{2+x}{2}-\frac{(1+x)}{2}\right], \mathcal{I}_{2}^{\mathrm{sq}}=\operatorname{Cos}\left(\left[x-\frac{1}{2}\right] \frac{\pi}{2}\right) \Gamma(1+x)\left[\frac{2 k_{1}}{1-x}-\frac{k_{1}}{2}-\frac{1+x}{4} k_{3}\right], \\
& \mathcal{I}_{3}^{\mathrm{sq}}= \frac{\left(k_{1} k_{3}\right)^{3}}{8} \frac{\Gamma(3+x)}{2} \operatorname{Cos}\left(\left[x-\frac{1}{2}\right] \frac{\pi}{2}\right), \mathcal{I}_{6}^{\mathrm{sq}}=\frac{k_{1} k_{3}^{2}}{8} \operatorname{Cos}\left(\left[x-\frac{1}{2}\right] \frac{\pi}{2}\right) \frac{(6+x) \Gamma(3+x)}{12} \\
& \mathcal{I}_{4}^{\mathrm{sq}}= \operatorname{Cos}\left(\left[x-\frac{1}{2}\right] \frac{\pi}{2}\right)\left\{\frac{k_{1} k_{3}^{2}}{2}\left[(3+x) \Gamma(1+x)-\Gamma(2+x) \frac{k_{3}}{2 k_{1}}\right]+\left(\overrightarrow{k_{1}} \cdot \vec{k}_{3}\right) k_{1}\left[(3+x) \Gamma(1+x)-\Gamma(2+x) \frac{1}{2}\right]\right\} \\
& \mathcal{I}_{5}^{\mathrm{sq}}= \operatorname{Cos}\left(\left[x-\frac{1}{2}\right] \frac{\pi}{2}\right)\left\{\frac{k_{1} k_{3}^{2}}{2}\left[\Gamma(1+x)+\Gamma(2+x) \frac{k_{3}}{2 k_{1}}\right]+\frac{\left(\vec{k}_{1} \cdot \vec{k}_{3}\right) k_{1}^{2}}{k_{1}}\left[\Gamma(1+x)+\Gamma(2+x) \frac{k_{1}}{K}\right]\right\} \\
& \mathcal{I}_{7}^{\mathrm{sq}}=\operatorname{Cos}\left(\left[x-\frac{1}{2}\right] \frac{\pi}{2}\right) \frac{2+x}{2}\left[\Gamma(1+x)+\Gamma(2+x)\left(\frac{1}{4}+(3+x) \frac{k_{3}}{8 k_{1}}\right)\right]\left\{\frac{k_{1} k_{3}^{2}}{2}+\left(\overrightarrow{k_{1}} \cdot \vec{k}_{3}\right) k_{1}\right\} \\
& \mathcal{I}_{8}^{\mathrm{sq}}=\operatorname{Cos}\left(\left[x-\frac{1}{2}\right] \frac{\pi}{2}\right)\left\{\frac{k_{1} k_{3}^{2}}{2}\left[(3+x) \Gamma(1+x)+(3+x) \Gamma(2+x) \frac{k_{3}}{2 k_{1}}-\Gamma(3+x) \frac{k_{3}^{2}}{4 k_{1}^{2}}\right]\right. \\
&\left.+\left(\vec{k}_{1} \cdot \vec{k}_{3}\right) k_{1}\left[(3+x) \Gamma(1+x)+(3+x) \Gamma(2+x) \frac{1}{2}-\Gamma(3+x) \frac{1}{4}\right]\right\} .
\end{aligned}
$$

B. Functions appearing in the one-scalar two-tensor correlation

The functional dependence of the coefficients appearing in the context of the one-scalar two-tensor correlation can be expressed as

$$
\begin{aligned}
& \left(\nabla_{1}\right)_{i j ; k l}^{u}=\sum_{p=1}^{6}\left\{\frac{\left[\mathcal{J}_{p}\left(\vec{k}_{1}, \vec{k}_{2}, \vec{k}_{3}\right)\right]_{i j ; k l}^{u}}{k_{1}^{\nu_{s}}\left(k_{2} k_{3}\right)^{\nu_{T}}}+\frac{\left[\mathcal{J}_{p}\left(\vec{k}_{2}, \vec{k}_{1}, \vec{k}_{3}\right)\right]_{i j ; k l}^{u}}{k_{2}^{\nu_{s}}\left(k_{1} k_{3}\right)^{\nu_{T}}}+\frac{\left[\mathcal{J}_{p}\left(\overrightarrow{k_{3}}, \vec{k}_{2}, \vec{k}_{1}\right)\right]_{i j ; k l}^{u}}{k_{3}^{\nu_{s}}\left(k_{2} k_{1}\right)^{\nu_{T}}}\right\} \frac{\Gamma\left(7+p-4 v_{T}-2 v_{s}\right)}{c_{s}^{2 v_{s}-\frac{p}{3}-\frac{7}{3}} c_{T}^{4 v_{T}-\frac{2 p}{3}-\frac{14}{3}} \underline{K}^{7+p-4 v_{T}-2 v_{s}}}, \\
& \left(\nabla_{2}\right)_{i j ; k l}^{u}=\frac{\left[\frac{\left(\overrightarrow{k_{1}} \cdot \overrightarrow{k_{2}}\right)}{\vec{k}_{1}^{s_{1}}\left(k_{2} k_{3}\right)^{v_{T}}}+\frac{\left(\overrightarrow{k_{2}} \cdot \overrightarrow{k_{3}}\right)}{k_{2}^{v_{s}}\left(k_{1} k_{3}\right)^{v_{T}}}+\frac{\left(\overrightarrow{k_{3} \cdot \vec{k}_{1}}\right)}{k_{3}^{\nu_{s}}\left(k_{2} k_{3}\right)^{v_{T}}}\right]}{\left(\frac{3}{2}-v_{T}\right)^{2} c_{T}^{2}} \sum_{p=1}^{4} H_{p} \frac{\Gamma\left(9+p-4 v_{T}-2 v_{s}\right)}{c_{s}^{2 v_{s}-\frac{p}{3}-3} c_{T}^{4 v_{T}-\frac{2 p}{3}-6} \underline{K}^{9+p-4 v_{T}-2 v_{s}}} \mathcal{N}_{i j, k l}^{u}, \\
& \left(\nabla_{3}\right)_{i j ; k l}^{u}=\frac{\left[\left(\vec{k}_{1} \cdot \vec{k}_{2}\right) Y_{123}+\left(\overrightarrow{k_{1}} \cdot \overrightarrow{k_{3}}\right) Y_{132}+\left(\overrightarrow{k_{2}} \cdot \vec{k}_{3}\right) Y_{213}+\left(\overrightarrow{k_{2}} \cdot \vec{k}_{1}\right) Y_{231}+\left(\overrightarrow{k_{3}} \cdot \vec{k}_{2}\right) Y_{312}+\left(\overrightarrow{k_{3}} \cdot \vec{k}_{1}\right) Y_{321}\right] \mathcal{N}_{i j, k l}^{u},}{\left(\frac{3}{2}-v_{T}\right)^{2} c_{T}^{2}}, \\
& \left(\nabla_{4}\right)_{i j ; k l}^{u}=\left(\frac{3}{2}-v_{s}\right)\left[\tilde{\mathcal{J}}_{123}+\tilde{\mathcal{J}}_{132}+\tilde{\mathcal{J}}_{213}+\tilde{\mathcal{J}}_{231}+\tilde{\mathcal{J}}_{312}+\tilde{\mathcal{J}}_{321}\right] \mathcal{N}_{i j, k l}^{u}, \\
& \left(\nabla_{5}\right)_{i j ; k l}^{u}=\left[\tilde{\mathcal{C}}_{123}+\tilde{\mathcal{C}}_{132}+\tilde{\mathcal{C}}_{213}+\tilde{\mathcal{C}}_{231}+\tilde{\mathcal{C}}_{312}+\tilde{\mathcal{C}}_{321}\right]_{i j, k l}^{u}, \\
& \left(\nabla_{6}\right)_{i j ; k l}^{u}=\left[\hat{\mathcal{W}}_{123}+\hat{\mathcal{W}}_{132}+\hat{\mathcal{W}}_{213}+\hat{\mathcal{W}}_{231}+\hat{\mathcal{W}}_{312}+\hat{\mathcal{W}}_{321}\right] \mathcal{N}_{i j, k l}^{u} \text {, } \\
& \left(\nabla_{7}\right)_{i j ; k l}^{u}=\left[k_{1 m} k_{1 m^{\prime}}\left\{\bar{X}_{123}+\bar{X}_{132}\right\}+k_{2 m} k_{2 m^{\prime}}\left\{\bar{X}_{231}+\bar{X}_{213}\right\}+k_{3 m} k_{3 m^{\prime}}\left\{\bar{X}_{312}+\bar{X}_{321}\right\}\right] \mathcal{N}_{i j, k l}^{u} \mathcal{N}_{m n, m^{\prime} n}^{u} \text {, }
\end{aligned}
$$


with

$$
\begin{aligned}
& {\left[\mathcal{J}_{1}\left(\vec{k}_{a}, \vec{k}_{b}, \vec{k}_{c}\right)\right]_{i j ; k l}^{u}=\mathcal{N}_{i j, k l}^{u}\left(\frac{3}{2}-v_{T}\right)^{2},\left[\mathcal{J}_{2}\left(\vec{k}_{a}, \vec{k}_{b}, \vec{k}_{c}\right)\right]_{i j ; k l}^{u}=\left[\mathcal{J}_{1}\left(\vec{k}_{a}, \vec{k}_{b}, \vec{k}_{c}\right)\right]_{i j ; k l}^{u} \underline{K} c_{s}^{-\frac{1}{3}} c_{T}^{-\frac{2}{3}},} \\
& {\left[\mathcal{J}_{3}\left(\vec{k}_{a}, \vec{k}_{b}, \vec{k}_{c}\right)\right]_{i j ; k l}^{u}=\mathcal{N}_{i j, k l}^{u}\left(\frac{3}{2}-v_{T}\right)\left[\left(k_{a}^{2}+k_{b}^{2}+k_{a} k_{b}\right)+\left(\frac{3}{2}-v_{T}\right) k_{a}\left(k_{b}+k_{c}\right)\right]} \\
& {\left[\mathcal{J}_{4}\left(\vec{k}_{a}, \vec{k}_{b}, \vec{k}_{c}\right)\right]_{i j ; k l}^{u}=\mathcal{N}_{i j, k l}^{u}\left(\frac{3}{2}-v_{T}\right)\left[k_{b} k_{c}\left(k_{b}+k_{c}\right)+k_{a}\left(k_{b}^{2}+k_{c}^{2}+k_{b} k_{c}\right)\right],} \\
& {\left[\mathcal{J}_{5}\left(\vec{k}_{a}, \vec{k}_{b}, \vec{k}_{c}\right)\right]_{i j ; k l}^{u}=\mathcal{N}_{i j, k l}^{u}\left[k_{b}^{2} k_{c}^{2}+k_{a} k_{b} k_{c}\left(k_{b}+k_{c}\right)\right],\left[\mathcal{J}_{6}\left(\vec{k}_{a}, \vec{k}_{b}, \vec{k}_{c}\right)\right]_{i j ; k l}^{u}=i \mathcal{N}_{i j, k l}^{u} k_{a} k_{b}^{2} k_{c}^{2},}
\end{aligned}
$$

(includes 3 permutations of $a, b, c$ ),

$$
Y_{a b c}=\frac{1}{k_{a}^{\nu_{s}}\left(k_{b} k_{c}\right)^{\nu_{T}}}\left\{\sum_{p=1}^{4} H_{p} \frac{\Gamma\left(9+p-4 v_{T}-2 v_{s}\right)}{c_{s}^{2 v_{s}-\frac{p}{3}-3} c_{T}^{4 v_{T}-\frac{2 p}{3}-6} \underline{K}^{9+p-4 v_{T}-2 v_{s}}}+\sum_{q=1}^{5} \mathcal{A}_{q}^{a b c} \frac{\Gamma\left(8+q-4 v_{T}-2 v_{s}\right)}{c_{s}^{2 v_{s}-\frac{q}{3}-\frac{8}{3}} c_{T}^{4 v_{T}-\frac{2 q}{3}-\frac{16}{3}} \underline{K}^{8+q-4 v_{T}-2 v_{s}}}\right\}
$$

(with $a, b, c=1,2,3$ with $a \neq b \neq c$ ),

$H_{1}=H_{2}=L_{1}, H_{3}=\frac{H_{1}\left(k_{a} k_{b}+k_{b} k_{c}+k_{c} k_{a}\right)}{c_{s}^{-\frac{2}{3}} c_{T}^{-\frac{4}{3}} \underline{K}^{2}}, H_{4}=-\frac{k_{a} k_{b} k_{c}}{c_{s}^{-1} c_{T}^{-2} \underline{K}^{3}}$,

$\mathcal{A}_{1}^{a b c}=\frac{a^{2} Y_{s} c_{s}^{2}}{t_{1}}\left(\frac{3}{2}-v_{s}\right)^{2}, \mathcal{A}_{2}^{a b c}=c_{s}^{-\frac{1}{3}} c_{T}^{-\frac{2}{3}} \underline{K} \mathcal{A}_{1}^{a b c}, \mathcal{A}_{3}^{a b c}=-\left(k_{a} k_{b}+k_{b} k_{c}+k_{c} k_{a}+k_{a}^{2}\right) \mathcal{A}_{1}^{a b c}$,

$\mathcal{A}_{4}^{a b c}=-\frac{a^{2} Y_{s} c_{s}^{2}}{t_{1}}\left(\frac{3}{2}-v_{s}\right)\left[k_{a} k_{b} k_{c}\left(\frac{3}{2}-v_{s}\right)+k_{a}^{2}\left(k_{b}+k_{c}\right)\right], \mathcal{A}_{5}^{a b c}=\frac{a^{2} Y_{s} c_{s}^{2}}{t_{1}} k_{a}^{2} k_{b} k_{c}$,

$\left[\tilde{\mathcal{C}}_{a b c}\right]_{i j ; k l}^{u}=\sum_{p=1}^{6} \frac{\left[\mathcal{J}_{p}\left(\overrightarrow{k_{a}}, \overrightarrow{k_{b}}, \vec{k}_{c}\right)\right]_{i j ; k l}^{u}}{k_{a}^{\nu_{s}}\left(k_{b} k_{c}\right)^{\nu_{T}}} \frac{\Gamma\left(7+p-4 v_{T}-2 v_{s}\right)}{c_{s}^{2 v_{s}-\frac{p}{3}-\frac{7}{3}} c_{T}^{4 v_{T}-\frac{2 p}{3}-\frac{14}{3}} \underline{K}^{7+p-4 v_{T}-2 v_{s}}}$ (includes 6 permuations of $a, b, c)$,

$\hat{\mathcal{W}}_{a b c}=\frac{1}{k_{a}^{v_{s}}\left(k_{b} k_{c}\right)^{v_{T}}}\left\{k_{a}^{2} \frac{\Gamma\left(8-4 v_{T}-2 v_{s}\right)}{c_{s}^{2 v_{s}-\frac{8}{3}} c_{T}^{4 v_{T}-\frac{16}{3}} \underline{K}^{8-4 v_{T}-2 v_{s}}}+\frac{a^{2} Y_{s} c_{s}^{2}}{t_{1}} \sum_{p=1}^{7} a_{p}^{a b c} \frac{\Gamma\left(6+p-4 v_{T}-2 v_{s}\right)}{c_{S}^{2 v_{s}-\frac{p}{3}-2} c_{T}^{4 v_{T}-\frac{2 p}{3}-4} \underline{K}^{6+p-4 v_{T}-2 v_{s}}}\right\}$,

$\bar{X}_{a b c}=\sum_{p=1}^{7} \frac{a_{p}^{a b c}}{k_{a}^{\nu_{s}}\left(k_{b} k_{c}\right)^{\nu_{T}}} \frac{\Gamma\left(7+p-4 v_{T}-2 v_{s}\right)}{c_{s}^{2 v_{s}-\frac{p}{3}-\frac{7}{3}} c_{T}^{4 v_{T}-\frac{2 p}{3}-\frac{14}{3}} \underline{K}^{7+p-4 v_{T}-2 v_{s}}}$

$\tilde{\mathcal{J}}_{a b c}=\sum_{p=1}^{7} \frac{a_{p}^{a b c}}{k_{a}^{\nu_{s}}\left(k_{b} k_{c}\right)^{v_{T}}} \frac{\Gamma\left(6+p-4 v_{T}-2 v_{s}\right)}{c_{s}^{2 v_{s}-\frac{p}{3}-2 v_{T}-\frac{2 p}{3}-4} \underline{K}_{T}^{6+p-4 v_{T}-2 v_{s}}}, a_{1}^{a b c}=\left(\frac{3}{2}-v_{T}\right)^{2}\left(\frac{3}{2}-v_{s}\right), a_{2}^{a b c}=a_{1}^{a b c} c_{s}^{-\frac{1}{3}} c_{T}^{-\frac{2}{3}} \underline{K}$,

$a_{3}^{a b c}=\left(\frac{3}{2}-v_{T}\right)\left(\frac{3}{2}-v_{s}\right)\left[k_{a}\left(k_{b}+k_{c}\right)+k_{b}^{2}+k_{c}^{2}+k_{b} k_{c}\right]+\left(\frac{3}{2}-v_{T}\right)^{2} k_{a}^{2}$,

$a_{4}^{a b c}=\left[k_{a}^{2}\left(k_{b}+k_{c}\right)\left(\frac{3}{2}-v_{T}\right)+\left\{k_{a}\left(k_{b}^{2}+k_{c}^{2}+k_{b} k_{c}\right)+k_{b} k_{c}\left(k_{b}+k_{c}\right)\right\}\left(\frac{3}{2}-v_{T}\right)\left(\frac{3}{2}-v_{s}\right)\right]$,

$a_{5}^{a b c}=\left[\left(\frac{3}{2}-v_{T}\right) k_{a}^{2}\left(k_{b}^{2}+k_{c}^{2}+k_{b} k_{c}\right)+\left(\frac{3}{2}-v_{s}\right) k_{b}^{2} k_{c}^{2}+\left(\frac{3}{2}-v_{T}\right)\left(\frac{3}{2}-v_{s}\right) k_{a} k_{b} k_{c}\left(k_{b}+k_{c}\right)\right]$,

$a_{6}^{a b c}=k_{a} k_{b} k_{c}\left[\left(\frac{3}{2}-v_{T}\right) k_{b} k_{c}+\left(\frac{3}{2}-v_{s}\right) k_{a}\left(k_{b}+k_{c}\right)\right], a_{7}^{a b c}=-k_{a}^{2} k_{b}^{2} k_{c}^{2}$.

After using the basis transformation mentioned in Eq. (3.23) the reduced form of the above mentioned coefficients can be expressed in the following form: 


$$
\begin{aligned}
& \left(\nabla_{1}\right)^{u ; \lambda_{2} ; \lambda_{3}}=\sum_{p=1}^{6}\left\{\frac{\left[\mathcal{J}_{p}\left(\vec{k}_{1}, \vec{k}_{2}, \vec{k}_{3}\right)\right]^{u ; \lambda_{2} ; \lambda_{3}}}{k_{1}^{\nu_{s}}\left(k_{2} k_{3}\right)^{\nu_{T}}}+\frac{\left[\mathcal{J}_{p}\left(\vec{k}_{2}, \vec{k}_{1}, \vec{k}_{3}\right)\right]^{u ; \lambda_{2} ; \lambda_{3}}}{k_{2}^{\nu_{s}}\left(k_{1} k_{3}\right)^{\nu_{T}}}+\frac{\left[\mathcal{J}_{p}\left(\vec{k}_{3}, \vec{k}_{2}, \vec{k}_{1}\right)\right]^{u ; \lambda_{2} ; \lambda_{3}}}{k_{3}^{\nu_{s}}\left(k_{2} k_{1}\right)^{\nu_{T}}}\right\} \\
& \times \frac{\Gamma\left(7+p-4 v_{T}-2 v_{s}\right)}{c_{S}^{2 v_{s}-\frac{p}{3}-\frac{7}{3}} c_{T}^{4 v_{T}-\frac{2 p}{3}-\frac{14}{3}} \underline{K}^{7+p-4 v_{T}-2 v_{s}}}, \\
& \left(\nabla_{2}\right)^{u ; \lambda_{2} ; \lambda_{3}}=\frac{2\left[\frac{\left(\overrightarrow{k_{1}} \cdot \overrightarrow{k_{2}}\right)}{k_{1}^{v_{s}}\left(k_{2} k_{3}\right)^{v_{T}}}+\frac{\left(\overrightarrow{k_{2}} \cdot \overrightarrow{k_{3}}\right)}{k_{2}^{s_{s}}\left(k_{1} k_{3}\right)^{v_{T}}}+\frac{\left(\overrightarrow{k_{3}} \cdot \overrightarrow{k_{1}}\right)}{k_{3}^{v_{s}}\left(k_{1} k_{2}\right)^{v_{T}}}\right]^{u ; \lambda_{2} ; \lambda_{3}}}{\left(\frac{3}{2}-v_{T}\right)^{2} c_{T}^{2}} \sum_{p=1}^{4} H_{p} \frac{\Gamma\left(9+p-4 v_{T}-2 v_{s}\right)}{c_{s}^{2 v_{s}-\frac{p}{3}-3} c_{T}^{4 v_{T}-\frac{2 p}{3}-6} \underline{K}^{9+p-4 v_{T}-2 v_{s}}}, \\
& \left(\nabla_{3}\right)^{u ; \lambda_{2} ; \lambda_{3}}=\frac{2\left[\left(\overrightarrow{k_{1}} \cdot \overrightarrow{k_{2}}\right) Y_{123}+\left(\overrightarrow{k_{1}} \cdot \overrightarrow{k_{3}}\right) Y_{132}+\left(\overrightarrow{k_{2}} \cdot \overrightarrow{k_{3}}\right) Y_{213}+\left(\overrightarrow{k_{2}} \cdot \overrightarrow{k_{1}}\right) Y_{231}+\left(\overrightarrow{k_{3}} \cdot \overrightarrow{k_{2}}\right) Y_{312}+\left(\overrightarrow{k_{3}} \cdot \overrightarrow{k_{1}}\right) Y_{321}\right]^{u ; \lambda_{2} ; \lambda_{3}}}{\left(\frac{3}{2}-v_{T}\right)^{2} c_{T}^{2}}, \\
& \left(\nabla_{4}\right)^{u ; \lambda_{2} ; \lambda_{3}}=2\left(\frac{3}{2}-v_{s}\right)\left[\tilde{\mathcal{J}}_{123}+\tilde{\mathcal{J}}_{132}+\tilde{\mathcal{J}}_{213}+\tilde{\mathcal{J}}_{231}+\tilde{\mathcal{J}}_{312}+\tilde{\mathcal{J}}_{321}\right] \delta^{\lambda_{2} \lambda_{3}}, \\
& \left(\nabla_{5}\right)^{u ; \lambda_{2} ; \lambda_{3}}=\left[\tilde{\mathcal{C}}_{123}+\tilde{\mathcal{C}}_{132}+\tilde{\mathcal{C}}_{213}+\tilde{\mathcal{C}}_{231}+\tilde{\mathcal{C}}_{312}+\tilde{\mathcal{C}}_{321}\right]^{u ; \lambda_{2} ; \lambda_{3}}, \\
& \left(\nabla_{6}\right)^{u ; \lambda_{2} ; \lambda_{3}}=2\left[\hat{\mathcal{W}}_{123}+\hat{\mathcal{W}}_{132}+\hat{\mathcal{W}}_{213}+\hat{\mathcal{W}}_{231}+\hat{\mathcal{W}}_{312}+\hat{\mathcal{W}}_{321}\right] \delta^{\lambda_{2} \lambda_{3}}, \\
& \left(\nabla_{7}\right)^{u ; \lambda_{2} ; \lambda_{3}}=\left[Z_{1}^{u ; \lambda_{2} ; \lambda_{3}}\left\{\bar{X}_{123}+\bar{X}_{132}\right\}+Z_{2}^{u ; \lambda_{2} ; \lambda_{3}}\left\{\bar{X}_{231}+\bar{X}_{213}\right\}+Z_{3}^{u ; \lambda_{2} ; \lambda_{3}}\left\{\bar{X}_{312}+\bar{X}_{321}\right\}\right],
\end{aligned}
$$

with

$$
\begin{aligned}
& {\left[\mathcal{J}_{1}\left(\vec{k}_{a}, \vec{k}_{b}, \vec{k}_{c}\right)\right]^{u ; \lambda_{2} ; \lambda_{3}}=2\left(\frac{3}{2}-v_{T}\right)^{2},\left[\mathcal{J}_{2}\left(\vec{k}_{a}, \vec{k}_{b}, \vec{k}_{c}\right)\right]^{u ; \lambda_{2} ; \lambda_{3}}=\left[\mathcal{J}_{1}\left(\vec{k}_{a}, \vec{k}_{b}, \vec{k}_{c}\right)\right]^{u ; \lambda_{2} ; \lambda_{3}} c_{s}^{-\frac{1}{3}} c_{T}^{-\frac{2}{3}} \underline{K},} \\
& {\left[\mathcal{J}_{3}\left(\overrightarrow{k_{a}}, \vec{k}_{b}, \vec{k}_{c}\right)\right]^{u ; \lambda_{2} ; \lambda_{3}}=2 \lambda_{2} \lambda_{3}\left(\frac{3}{2}-v_{T}\right)\left[\left(k_{a}^{2}+k_{b}^{2}+k_{a} k_{b}\right)+\left(\frac{3}{2}-v_{T}\right) k_{a}\left(k_{b}+k_{c}\right)\right]} \\
& {\left[\mathcal{J}_{4}\left(\vec{k}_{a}, \vec{k}_{b}, \vec{k}_{c}\right)\right]^{u ; \lambda_{2} ; \lambda_{3}}=2\left(\frac{3}{2}-v_{T}\right)\left[\lambda_{2}^{3} k_{b} k_{c}\left(k_{b}+k_{c}\right)+\lambda_{3}^{3} k_{a}\left(k_{b}^{2}+k_{c}^{2}+k_{b} k_{c}\right)\right],} \\
& {\left[\mathcal{J}_{5}\left(\vec{k}_{a}, \vec{k}_{b}, \vec{k}_{c}\right)\right]^{u ; \lambda_{2} ; \lambda_{3}}=2 \lambda_{2}^{2} \lambda_{3}^{2}\left[k_{b}^{2} k_{c}^{2}+k_{a} k_{b} k_{c}\left(k_{b}+k_{c}\right)\right],\left[\mathcal{J}_{6}\left(\vec{k}_{a}, \vec{k}_{b}, \vec{k}_{c}\right)\right]^{u ; \lambda_{2} ; \lambda_{3}}=2 i \lambda_{3}^{2} \lambda_{2}^{2} k_{a} k_{b}^{2} k_{c}^{2},}
\end{aligned}
$$

(includes 3 permutations of $a, b, c$ ),

$\left[\tilde{\mathcal{C}}_{a b c}\right]^{u ; \lambda_{2} ; \lambda_{3}}=\sum_{p=1}^{6} \frac{\left[\mathcal{J}_{p}\left(\vec{k}_{a}, \vec{k}_{b}, \vec{k}_{c}\right)\right]^{u ; \lambda_{2} ; \lambda_{3}}}{k_{a}^{\nu_{s}}\left(k_{b} k_{c}\right)^{\nu_{T}}} \frac{\Gamma\left(7+p-4 v_{T}-2 v_{s}\right)}{c_{s}^{2 v_{s}-\frac{p}{3}-\frac{7}{3}} c_{T}^{4 v_{T}-\frac{2 p}{3}-\frac{14}{3}} \underline{K}^{7+p-4 v_{T}-2 v_{s}}} \quad$ (includes 6 permuations of $\left.a, b, c\right)$,

$Z_{a}^{u ; \lambda_{2} ; \lambda_{3}}=\frac{c_{s}^{-\frac{1}{3}} c_{T}^{-\frac{2}{3}} \underline{K}}{32 k_{a}^{2} k_{b}^{2} k_{c}^{2}}\left(k_{a}-k_{b}-k_{c}\right)\left(k_{a}+k_{b}-k_{c}\right)\left(k_{a}-k_{b}+k_{c}\right)\left[k_{a}^{2}-\left(\lambda_{2} k_{b}+\lambda_{3} k_{c}\right)^{2}\right]^{u}$.

\section{Functions appearing in two scalar one tensor correlation}

The functional dependence of the coefficients appearing in the context of two scalar one tensor correlation can be expressed as 


$$
\begin{aligned}
& \left(\hat{\nabla}_{1}\right)_{i j}=\left[\frac{\left(k_{2 i} k_{3 j}+k_{3 i} k_{2 j}\right)}{k_{1}^{\nu_{T}}\left(k_{2} k_{3}\right)^{\nu_{s}}}+\frac{\left(k_{1 i} k_{3 j}+k_{3 i} k_{1 j}\right)}{k_{2}^{\nu_{T}}\left(k_{1} k_{3}\right)^{\nu_{s}}}+\frac{\left(k_{1 i} k_{2 j}+k_{2 i} k_{1 j}\right)}{k_{3}^{\nu_{T}}\left(k_{1} k_{2}\right)^{\nu_{s}}}\right] \tilde{O}, \\
& \left(\hat{\nabla}_{2}\right)_{i j}=c_{s}\left(\frac{3}{2}-v_{T}\right)\left[\frac{\left(k_{2 i} k_{3 j} P_{123}+k_{3 i} k_{2 j} P_{132}\right)}{k_{1}^{\nu_{T}}\left(k_{2} k_{3}\right)^{\nu_{s}}}+\frac{\left(k_{1 i} k_{3 j} P_{213}+k_{3 i} k_{1 j} P_{231}\right)}{k_{2}^{\nu_{T}}\left(k_{1} k_{3}\right)^{\nu_{s}}}+\frac{\left(k_{1 i} k_{2 j} P_{312}+k_{2 i} k_{1 j} P_{321}\right)}{k_{3}^{\nu_{T}}\left(k_{1} k_{2}\right)^{\nu_{s}}}\right], \\
& \left(\hat{\nabla}_{3}\right)_{i j}=c_{s}\left[\frac{\left(k_{2 i} k_{3 j} R_{123}+k_{3 i} k_{2 j} R_{132}\right)}{k_{1}^{\nu_{T}}\left(k_{2} k_{3}\right)^{\nu_{s}}}+\frac{\left(k_{1 i} k_{3 j} R_{213}+k_{3 i} k_{1 j} R_{231}\right)}{k_{2}^{\nu_{T}}\left(k_{1} k_{3}\right)^{\nu_{s}}}+\frac{\left(k_{1 i} k_{2 j} R_{312}+k_{2 i} k_{1 j} R_{321}\right)}{k_{3}^{\nu_{T}}\left(k_{1} k_{2}\right)^{\nu_{s}}}\right], \\
& \left(\hat{\nabla}_{4}\right)_{i j}=\left[k_{1}^{2} \frac{\left(k_{2 i} k_{3 j} \tilde{R}_{123}+k_{3 i} k_{2 j} \tilde{R}_{132}\right)}{k_{1}^{\nu_{T}}\left(k_{2} k_{3}\right)^{\nu_{s}}}+k_{2}^{2} \frac{\left(k_{1 i} k_{3 j} \tilde{R}_{213}+k_{3 i} k_{1 j} \tilde{R}_{231}\right)}{k_{2}^{\nu_{T}}\left(k_{1} k_{3}\right)^{\nu_{s}}}+k_{3}^{2} \frac{\left(k_{1 i} k_{2 j} \tilde{R}_{312}+k_{2 i} k_{1 j} \tilde{R}_{321}\right)}{k_{3}^{\nu_{T}}\left(k_{1} k_{2}\right)^{\nu_{s}}}\right], \\
& \left(\hat{\nabla}_{5}\right)_{i j}=\left[k_{1}^{2} \frac{\left(k_{2 i} k_{3 j}+k_{3 i} k_{2 j}\right)}{k_{1}^{\nu_{T}}\left(k_{2} k_{3}\right)^{\nu_{s}}}+k_{2}^{2} \frac{\left(k_{1 i} k_{3 j}+k_{3 i} k_{1 j}\right)}{k_{2}^{\nu_{T}}\left(k_{1} k_{3}\right)^{\nu_{s}}}+k_{3}^{2} \frac{\left(k_{1 i} k_{2 j}+k_{2 i} k_{1 j}\right)}{k_{3}^{\nu_{T}}\left(k_{1} k_{2}\right)^{\nu_{s}}}\right] \tilde{O}, \\
& \left(\hat{\nabla}_{6}\right)_{i j}=\left[k_{1}^{2} \frac{\left(k_{2 i} k_{3 j} \tilde{L}_{123}+k_{3 i} k_{2 j} \tilde{L}_{132}\right)}{k_{1}^{\nu_{T}}\left(k_{2} k_{3}\right)^{\nu_{s}}}+k_{2}^{2} \frac{\left(k_{1 i} k_{3 j} \tilde{L}_{213}+k_{3 i} k_{1 j} \tilde{L}_{231}\right)}{k_{2}^{\nu_{T}}\left(k_{1} k_{3}\right)^{\nu_{s}}}+k_{3}^{2} \frac{\left(k_{1 i} k_{2 j} \tilde{L}_{312}+k_{2 i} k_{1 j} \tilde{L}_{321}\right)}{k_{3}^{\nu_{T}}\left(k_{1} k_{2}\right)^{\nu_{s}}}\right] .
\end{aligned}
$$

with

$$
\begin{aligned}
& \tilde{O}=\left\{\sum_{p=1}^{4} O_{p} \frac{\Gamma\left(9+p-4 v_{s}-2 v_{T}\right)}{c_{s}^{4 v_{s}-\frac{2 p}{3}-6} c_{T}^{2 v_{T}-\frac{p}{3}-3} \underline{K}^{9+p-4 v_{s}-2 v_{T}}}\right\} \\
& O_{1}=1, O_{2}=i c_{s}^{-\frac{2}{3}} c_{T}^{-\frac{1}{3}} \underline{\underline{K}}, \\
& O_{3}=-\left(k_{a} k_{b}+k_{b} k_{c}+k_{c} k_{a}\right), O_{4}=-i k_{a} k_{b} k_{c} \\
& P_{a b c}=\sum_{p=1}^{5} m_{p}^{a b c} \frac{\Gamma\left(8+p-4 v_{T}-2 v_{s}\right)}{c_{s}^{4 v_{s}-\frac{2 p}{3}-\frac{16}{3}} c_{T}^{2 v_{T}-\frac{p}{3}-\frac{8}{3}} \underline{K}^{8+p-4 v_{s}-2 v_{T}}} \\
& m_{1}^{a b c}=\left(\frac{3}{2}-v_{T}\right), m_{2}^{a b c}=c_{s}^{-\frac{2}{3}} c_{T}^{-\frac{1}{3}} \underline{\underline{K}} m_{1}^{a b c} \\
& m_{3}^{a b c}=\left[\left(\frac{3}{2}-v_{T}\right)\left(k_{a} k_{b}+k_{b} k_{c}+k_{c} k_{a}\right)+k_{a}^{2}\right], m_{4}^{a b c} \\
& =\left[\left(\frac{3}{2}-v_{T}\right) k_{a} k_{b} k_{c}+k_{a}^{2}\left(k_{b}+k_{c}\right)\right], m_{5}^{a b c}=k_{a}^{2} k_{b} k_{c}
\end{aligned}
$$




$$
\begin{aligned}
& {\left[\hat{\mathcal{J}}_{1}\left(\vec{k}_{a}, \vec{k}_{b}, \vec{k}_{c}\right)\right]^{u}=\left(\frac{3}{2}-v_{s}\right)\left(\frac{3}{2}-v_{T}\right),\left[\hat{\mathcal{J}}_{2}\left(\vec{k}_{a}, \vec{k}_{b}, \vec{k}_{c}\right)\right]^{u}=i c_{s}^{-\frac{2}{3}} c_{T}^{-\frac{1}{3}} \underline{\underline{\underline{T}}}\left[\hat{\mathcal{J}}_{1}\left(\vec{k}_{a}, \vec{k}_{b}, \vec{k}_{c}\right)\right]^{u},} \\
& {\left[\hat{\mathcal{J}}_{3}\left(\overrightarrow{k_{a}}, \vec{k}_{b}, \vec{k}_{c}\right)\right]^{u}=-\left[\left(\frac{3}{2}-v_{s}\right) k_{a}^{2}+\left(\frac{3}{2}-v_{T}\right) k_{b}^{2}+\left(\frac{3}{2}-v_{s}\right)\left(\frac{3}{2}-v_{T}\right)\left\{k_{a} k_{b}+k_{b} k_{c}+k_{c} k_{a}\right\}\right],} \\
& {\left[\hat{\mathcal{J}}_{4}\left(\vec{k}_{a}, \vec{k}_{b}, \vec{k}_{c}\right)\right]^{u}=-i\left[\left(\frac{3}{2}-v_{s}\right) k_{a}^{2} k_{c}+\left(\frac{3}{2}-v_{T}\right) k_{c}^{2} k_{a}+\left(\frac{3}{2}-v_{s}\right) k_{a}^{2} k_{b}\right.} \\
& \left.+\left(\frac{3}{2}-v_{T}\right) k_{c}^{2} k_{b}+k_{a} k_{b} k_{c}\left[\hat{\mathcal{J}}_{1}\left(\vec{k}_{a}, \vec{k}_{b}, \vec{k}_{c}\right)\right]^{u}\right], \\
& {\left[\hat{\mathcal{J}}_{5}\left(\vec{k}_{a}, \vec{k}_{b}, \vec{k}_{c}\right)\right]^{u}=\left[k_{a}^{2} k_{c}^{2}+\left(\frac{3}{2}-v_{s}\right) k_{a}^{2} k_{b} k_{c}+\left(\frac{3}{2}-v_{T}\right) k_{a} k_{b} k_{c}^{2}\right],\left[\hat{\mathcal{J}}_{6}\left(\vec{k}_{a}, \vec{k}_{b}, \vec{k}_{c}\right)\right]^{u}=i k_{a}^{2} k_{b} k_{c}^{2},} \\
& \overline{\mathcal{A}}_{1}^{a b c}=\left(\frac{3}{2}-v_{T}\right), \overline{\mathcal{A}}_{2}^{a b c}=i c_{s}^{-\frac{2}{3}} c_{T}^{-\frac{1}{3}} \underline{\underline{K}} \overline{\mathcal{A}}_{1}^{a b c}, \overline{\mathcal{A}}_{3}^{a b c}=-\left(\frac{3}{2}-v_{T}\right)\left[k_{a} k_{b}+k_{b} k_{c}+k_{c} k_{a}+k_{a}^{2}\right], \\
& \overline{\mathcal{A}}_{4}^{a b c}=\left[k_{a} k_{b} k_{c}\left(\frac{3}{2}-v_{T}\right)+k_{a}^{2}\left(k_{b}+k_{c}\right)\right], \overline{\mathcal{A}}_{5}^{a b c}=k_{a}^{2} k_{b} k_{c} \\
& \tilde{R}_{a b c}=k_{a}^{2} L_{1} \tilde{O}+\frac{a^{2} Y_{s}}{t_{1}} \frac{k_{a}^{2}}{k_{b}^{2}}\left(\frac{3}{2}-v_{s}\right) P_{a b c} \\
& L_{a b c}=L_{1}^{2} \tilde{O}-\frac{L_{1} a^{2} Y_{s}\left(\frac{3}{2}-v_{s}\right)}{t_{1}} \sum_{p=1}^{5} n_{q}^{a b c} \frac{\Gamma\left(8+p-4 v_{s}-2 v_{T}\right)}{c_{s}^{4 v_{s}-\frac{2 p}{3}-\frac{16}{3}} c_{T}^{2 v_{T}-\frac{p}{3}-\frac{8}{3}} \underline{K}^{8+p-4 v_{s}-2 v_{T}}} \\
& +\frac{a^{4} Y_{s}^{2}\left(\frac{3}{2}-v_{s}\right)^{2}}{t_{1}^{2} k_{b}^{2} k_{c}^{2}} \sum_{r=1}^{6} d_{r}^{a b c} \frac{\Gamma\left(7+p-4 v_{s}-2 v_{T}\right)}{c_{s}^{4 v_{s}-\frac{2 p}{3}-\frac{14}{3}} c_{T}^{2 v_{T}-\frac{p}{3}-\frac{7}{3}} \underline{\underline{K}}^{7+p-4 v_{s}-2 v_{T}}}, \\
& n_{1}^{a b c}=\left(\frac{3}{2}-v_{s}\right)\left(\frac{1}{k_{a}^{2}}+\frac{1}{k_{b}^{2}}\right), n_{2}^{a b c}=i c_{s}^{-\frac{2}{3}} c_{T}^{-\frac{1}{3}} \underline{\underline{K}} n_{1}^{a b c}, \\
& n_{3}^{a b c}=-\left[2+\left(\frac{3}{2}-v_{s}\right)\left(\frac{k_{c}}{k_{b}}+\frac{k_{b}}{k_{c}}\right)+\left(\frac{3}{2}-v_{s}\right) k_{a}^{2}\left(k_{b}+k_{c}\right)\left(\frac{1}{k_{a}^{2}}+\frac{1}{k_{b}^{2}}\right)\right] \text {, } \\
& n_{4}^{a b c}=-i\left\{\left(k_{c}+k_{b}\right)+k_{a}\left[2+\left(\frac{3}{2}-v_{s}\right)\left(\frac{k_{c}}{k_{b}}+\frac{k_{b}}{k_{c}}\right)\right]\right\}, n_{5}^{a b c}=k_{a}\left(k_{c}+k_{b}\right) \text {, } \\
& d_{1}^{a b c}=\left(\frac{3}{2}-v_{s}\right)^{2}, d_{2}^{a b c}=i c_{s}^{-\frac{2}{3}} c_{T}^{-\frac{1}{3}} \underline{\underline{K}} d_{1}^{a b c}, d_{3}^{a b c}=-\left(k_{b}^{2}+k_{c}^{2}+k_{b} k_{c}+k_{a} k_{b}+k_{a} k_{c}\right), d_{6}^{a b c}=i k_{a} k_{b}^{2} k_{c}^{2} \\
& d_{4}^{a b c}=-i\left[k_{b}^{2} k_{c}^{4}+\left(\frac{3}{2}-v_{s}\right)\left\{k_{b} k_{c}^{2}+k_{a}\left(k_{b}^{2}+k_{c}^{2}+k_{b} k_{c}\right)\right\}\right], d_{5}^{a b c}=\left[k_{b}^{2} k_{c}^{2}+k_{a}\left(k_{b}^{2} k_{c}+k_{b} k_{c}^{2}\left(\frac{3}{2}-v_{s}\right)\right)\right] \text {. }
\end{aligned}
$$


After using the basis transformation mentioned in Eq.

(3.23) we get

$$
\begin{aligned}
& \left(\hat{\nabla}_{1}\right)_{\lambda^{\prime}}=\left[\frac{\left(k_{2}^{\lambda^{\prime}} k_{3}^{\lambda^{\prime \prime}}+k_{3}^{\lambda^{\prime}} k_{2}^{\lambda^{\prime \prime}}\right)}{k_{1}^{\nu_{T}}\left(k_{2} k_{3}\right)^{\nu_{s}}}+\frac{\left(k_{1}^{\lambda^{\prime}} k_{3}^{\lambda^{\prime \prime}}+k_{3}^{\lambda^{\prime}} k_{1}^{\lambda^{\prime \prime}}\right)}{k_{2}^{\nu_{T}}\left(k_{1} k_{3}\right)^{v_{s}}}+\frac{\left(k_{1}^{\lambda^{\prime}} k_{2}^{\lambda^{\prime \prime}}+k_{2}^{\lambda^{\prime}} k_{1}^{\lambda^{\prime \prime}}\right)}{k_{3}^{\nu_{T}}\left(k_{1} k_{2}\right)^{v_{s}}}\right] \tilde{O} \delta_{\lambda, \lambda / \prime} \\
& \frac{\left(\hat{\nabla}_{2}\right)_{\lambda^{\prime}}}{c_{s}\left(\frac{3}{2}-v_{T}\right)}=\left[\frac{\left(k_{2}^{\lambda^{\prime}} k_{3}^{\lambda^{\prime \prime}} P_{123}+k_{3}^{\lambda^{\prime}} k_{2}^{\lambda^{\prime \prime}} P_{132}\right)}{k_{1}^{\nu_{T}}\left(k_{2} k_{3}\right)^{\nu_{s}}}+\frac{\left(k_{1}^{\lambda^{\prime}} k_{3}^{\lambda^{\prime \prime}} P_{213}+k_{3}^{\lambda^{\prime}} k_{1}^{\lambda^{\prime \prime}} P_{231}\right)}{k_{2}^{\nu_{T}}\left(k_{1} k_{3}\right)^{\nu_{s}}}+\frac{\left(k_{1}^{\lambda^{\prime}} k_{2}^{\lambda^{\prime \prime}} P_{312}+k_{2}^{\lambda^{\prime}} k_{1}^{\lambda^{\prime \prime}} P_{321}\right)}{k_{3}^{\nu_{T}}\left(k_{1} k_{2}\right)^{\nu_{s}}}\right] \delta_{\lambda, \lambda / \prime} \text {, } \\
& \left(\hat{\nabla}_{3}\right)_{\lambda^{\prime}}=c_{s}\left[\frac{\left(k_{2}^{\lambda^{\prime}} k_{3}^{\lambda^{\prime \prime}} R_{123}+k_{3}^{\lambda 6^{\prime}} k_{2}^{\lambda^{\prime \prime}} R_{132}\right)}{k_{1}^{\nu_{T}}\left(k_{2} k_{3}\right)^{v_{s}}}+\frac{\left(k_{1}^{\lambda^{\prime}} k_{3}^{\lambda^{\prime \prime}} R_{213}+k_{3}^{\lambda^{\prime \prime}} k_{1}^{\lambda^{\prime \prime}} R_{231}\right)}{k_{2}^{\nu_{T}}\left(k_{1} k_{3}\right)^{\nu_{s}}}+\frac{\left(k_{1}^{\lambda^{\prime}} k_{2}^{\lambda^{\prime \prime}} R_{312}+k_{2}^{\lambda^{\prime}} k_{1}^{\lambda^{\prime \prime}} R_{321}\right)}{k_{3}^{\nu_{T}}\left(k_{1} k_{2}\right)^{\nu_{s}}}\right] \delta_{\lambda, \lambda / 1}, \\
& \left(\hat{\nabla}_{4}\right)_{\lambda^{\prime}}=\left[k_{1}^{2} \frac{\left(k_{2}^{\lambda^{\prime}} k_{3}^{\lambda^{\prime \prime}} \tilde{R}_{123}+k_{3}^{\lambda^{\prime \prime}} k_{2}^{\lambda^{\prime \prime}} \tilde{R}_{132}\right)}{k_{1}^{\nu_{T}}\left(k_{2} k_{3}\right)^{\nu_{s}}}+k_{2}^{2} \frac{\left(k_{1}^{\lambda^{\prime}} k_{3}^{\lambda^{\prime \prime}} \tilde{R}_{213}+k_{3}^{\lambda^{\prime}} k_{1}^{\lambda^{\prime \prime}} \tilde{R}_{231}\right)}{k_{2}^{\nu_{T}}\left(k_{1} k_{3}\right)^{\nu_{s}}}+k_{3}^{2} \frac{\left(k_{1}^{\lambda^{\prime}} k_{2}^{\lambda^{\prime \prime}} \tilde{R}_{312}+k_{2} k_{1}^{\lambda^{\prime \prime}} \tilde{R}_{321}\right)}{k_{3}^{\nu_{T}}\left(k_{1} k_{2}\right)^{\nu_{s}}}\right] \delta_{\lambda, \lambda / \prime}, \\
& \left(\hat{\nabla}_{5}\right)_{\lambda^{\prime}}=\left[k_{1}^{2} \frac{\left(k_{2}^{\lambda^{\prime}} k_{3}^{\lambda^{\prime \prime}}+k_{3}^{\lambda^{\prime \prime}} k_{2}^{\lambda^{\prime \prime}}\right)}{k_{1}^{\nu_{T}}\left(k_{2} k_{3}\right)^{v_{s}}}+k_{2}^{2} \frac{\left(k_{1}^{\lambda^{\prime}} k_{3}^{\lambda^{\prime \prime}}+k_{3}^{\lambda^{\prime}} k_{1}^{\lambda^{\prime \prime}}\right)}{k_{2}^{\nu_{T}}\left(k_{1} k_{3}\right)^{v_{s}}}+k_{3}^{2} \frac{\left(k_{1}^{\lambda^{\prime}} k_{2}^{\lambda^{\prime \prime}}+k_{2}^{\lambda^{\prime}} k_{1}^{\lambda^{\prime \prime}}\right)}{k_{3}^{\nu_{T}}\left(k_{1} k_{2}\right)^{v_{s}}}\right] \tilde{O} \delta_{\lambda, \lambda, \prime}, \\
& \left(\hat{\nabla}_{6}\right)_{\lambda^{\prime}}=\left[k_{1}^{2} \frac{\left(k_{2}^{\lambda^{\prime}} k_{3}^{\lambda^{\prime \prime}} \tilde{L}_{123}+k_{3}^{\lambda^{\prime \prime}} k_{2}^{\lambda^{\prime \prime}} \tilde{L}_{132}\right)}{k_{1}^{\nu_{T}}\left(k_{2} k_{3}\right)^{\nu_{s}}}+k_{2}^{2} \frac{\left(k_{1}^{\lambda^{\prime}} k_{3}^{\lambda^{\prime \prime}} \tilde{L}_{213}+k_{3}^{\lambda^{\prime}} k_{1}^{\lambda^{\prime \prime}} \tilde{L}_{231}\right)}{k_{2}^{\nu_{T}}\left(k_{1} k_{3}\right)^{\nu_{s}}}+k_{3}^{2} \frac{\left(k_{1}^{\lambda^{\prime}} k_{2}^{\lambda^{\prime \prime}} \tilde{L}_{312}+k_{2}^{\lambda^{\prime}} k_{1}^{\lambda^{\prime \prime}} \tilde{L}_{321}\right)}{k_{3}^{\nu_{T}}\left(k_{1} k_{2}\right)^{\nu_{s}}}\right] \delta_{\lambda, \lambda / \prime},
\end{aligned}
$$

where $k_{i}^{\lambda}=k_{i}$ where $i=1,2,3$. Most surprisingly, the above coefficients are independent of $\lambda$ due to there being no parity violation.

D. Functions appearing in three-tensor correlation

The functional dependence of the coefficients appearing in the context of three-tensor correlation can be expressed as

$$
\begin{aligned}
& \Delta_{i_{1} j_{1} i_{2} j_{2} i_{3} j_{3}}^{(1)}=\frac{\sigma}{12} \mathcal{N}_{i_{1} j_{1} ; i j} \mathcal{N}_{i_{2} j_{2} ; j k} \mathcal{N}_{i_{3} j_{3} ; k i} c_{s}^{3}\left(\frac{3}{2}-v_{T}\right)^{3}\left[M_{123}+M_{132}+M_{213}+M_{231}+M_{312}+M_{321}\right] \\
& \Delta_{i_{1} j_{1} i_{2} j_{2} i_{3} j_{3}}^{(2)}=\frac{Y_{T}}{2 c_{T}^{2}} \mathcal{N}_{i_{1} j_{1} ; i k} \mathcal{N}_{i_{2} j_{2} ; j l} \mathcal{N}_{i_{3} j_{3} ; i j}\left[k_{3 k} k_{3 l}+k_{2 k} k_{2 l}+k_{1 k} k_{1 l}\right] \mathcal{Q} \\
& \Delta_{i_{1} j_{1} i_{2} j_{2} i_{3} j_{3}}^{(3)}=-\frac{Y_{T}}{2 c_{T}^{2}} \mathcal{N}_{i_{1} j_{1} ; i_{3} j_{3}} \mathcal{N}_{i_{2} j_{2} ; k l}\left[k_{3 k} k_{3 l}+k_{2 k} k_{2 l}+k_{1 k} k_{1 l}\right] \mathcal{Q}
\end{aligned}
$$

with

$$
\begin{aligned}
& \mathcal{Q}=\sum_{p=1}^{4} O_{p} \frac{\Gamma\left(9+p-6 v_{T}\right)}{K^{9+p-6 v_{T}}}, M_{a b c}=\sum_{p=1}^{7} b_{p}^{a b c} \frac{\Gamma\left(6+p-6 v_{T}\right)}{K^{6+p-6 v_{T}}}, b_{1}^{a b c}=\left(\frac{3}{2}-v_{T}\right)^{3}, b_{2}^{a b c}=b_{1}^{a b c} K \\
& b_{3}^{a b c}=\left(\frac{3}{2}-v_{T}\right)^{2}\left[k_{a}\left(k_{b}+k_{c}\right)+k_{b}^{2}+k_{c}^{2}+k_{b} k_{c}+k_{a}^{2}\right] \text {, } \\
& b_{4}^{a b c}=\left[k_{a}^{2}\left(k_{b}+k_{c}\right)\left(\frac{3}{2}-v_{T}\right)+\left\{k_{a}\left(k_{b}^{2}+k_{c}^{2}+k_{b} k_{c}\right)+k_{b} k_{c}\left(k_{b}+k_{c}\right)\right\}\left(\frac{3}{2}-v_{T}\right)^{2}\right], \\
& b_{5}^{a b c}=\left[\left(\frac{3}{2}-v_{T}\right)\left\{k_{a}^{2}\left(k_{b}^{2}+k_{c}^{2}+k_{b} k_{c}\right)+k_{b}^{2} k_{c}^{2}\right\}+\left(\frac{3}{2}-v_{T}\right)^{2} k_{a} k_{b} k_{c}\left(k_{b}+k_{c}\right)\right] \text {, } \\
& b_{6}^{a b c}=\left(\frac{3}{2}-v_{T}\right) k_{a} k_{b} k_{c}\left[k_{b} k_{c}+k_{a}\left(k_{b}+k_{c}\right)\right], b_{7}^{a b c}=-k_{a}^{2} k_{b}^{2} k_{c}^{2} \text {. }
\end{aligned}
$$


After using the basis transformation mentioned in Eq. (3.23) the helicity dependent functions are given by

$$
\begin{aligned}
& \Delta_{\lambda_{1} \lambda_{2} \lambda_{3}}^{(1)}=\frac{\sigma}{12} \delta_{\lambda_{1} \lambda^{\prime}} \delta_{\lambda_{2} \lambda^{\prime \prime}} \delta_{\lambda_{3} \lambda^{\prime \prime \prime}} c_{s}^{3}\left(\frac{3}{2}-v_{T}\right)^{3}\left[M_{123}^{\lambda^{\prime} \lambda^{\prime \prime} \lambda^{\prime \prime \prime}}+M_{132}^{\lambda^{\prime} \lambda^{\prime \prime} \lambda^{\prime \prime \prime}}+M_{213}^{\lambda^{\prime} \lambda^{\prime \prime} \lambda^{\prime \prime \prime}}+M_{231}^{\lambda^{\prime} \lambda^{\prime \prime} \lambda^{\prime \prime \prime}}+M_{312}^{\lambda^{\prime} \lambda^{\prime \prime} \lambda^{\prime \prime \prime}}+M_{32}^{\lambda^{\prime} \lambda^{\prime \prime} \lambda^{\prime \prime \prime}}\right], \\
& \Delta_{\lambda_{1} \lambda_{2} \lambda_{3}}^{(2)}=\frac{Y_{T}}{2 c_{T}^{2}} \delta_{\lambda_{1} \lambda^{\prime}} \delta_{\lambda_{2} \lambda^{\prime \prime}} \delta_{\lambda_{3} \lambda^{\prime \prime \prime}}\left[k_{3}^{\lambda^{\prime}} k_{3}^{\lambda^{\prime \prime}}+k_{2}^{\lambda^{\prime}} k_{2}^{\lambda^{\prime \prime}}+k_{1}^{\lambda^{\prime}} k_{1}^{\lambda^{\prime \prime}}\right] \mathcal{Q}^{\lambda^{\prime \prime \prime}} \\
& \Delta_{\lambda_{1} \lambda_{2} \lambda_{3}}^{(3)}=-\frac{Y_{T}}{2 c_{T}^{2}} \delta_{\lambda^{\prime \prime} \lambda^{\prime}} \delta_{\lambda_{2} \lambda_{1}} \delta_{\lambda_{3} \lambda^{\prime \prime \prime}}\left[k_{3}^{\lambda^{\prime \prime \prime}} k_{3}^{\lambda^{\prime \prime}}+k_{2}^{\lambda^{\prime \prime \prime}} k_{2}^{\lambda^{\prime \prime}}+k_{1}^{\lambda^{\prime \prime \prime}} k_{1}^{\lambda^{\prime \prime}}\right] \mathcal{Q},
\end{aligned}
$$

with

$$
\begin{aligned}
& M_{a b c}^{\lambda^{\prime} \lambda^{\prime \prime} \lambda^{\prime \prime \prime}}=\sum_{p=1}^{7}\left(b_{p}^{a b c}\right)^{\lambda^{\prime} \lambda^{\prime \prime} \lambda^{\prime \prime \prime}} \frac{\Gamma\left(6+p-6 v_{T}\right)}{K^{6+p-6 v_{T}}}, \mathcal{Q}^{\lambda^{\prime \prime \prime}}=\sum_{p=1}^{4} O_{p}^{\lambda^{\prime \prime \prime}} \frac{\Gamma\left(9+p-6 v_{T}\right)}{K^{9+p-6 v_{T}}}, \\
& \left(b_{1}^{a b c}\right)^{\lambda^{\prime} \lambda^{\prime \prime} \lambda^{\prime \prime \prime}}=\left(\frac{3}{2}-v_{T}\right)^{3},\left(b_{2}^{a b c}\right)^{\lambda^{\prime} \lambda^{\prime \prime} \lambda^{\prime \prime \prime}}=\left(b_{1}^{a b c}\right)^{\lambda^{\prime} \lambda^{\prime \prime} \lambda^{\prime \prime \prime}} K, \\
& \left(b_{3}^{a b c}\right)^{\lambda^{\prime} \lambda^{\prime \prime} \lambda^{\prime \prime \prime}}=\left(\frac{3}{2}-v_{T}\right)^{2}\left[k_{a}^{\lambda^{\prime}}\left(k_{b}^{\lambda^{\prime \prime}}+k_{c}^{\lambda^{\prime \prime \prime}}\right)+\left(k_{b}^{\lambda^{\prime \prime}}\right)^{2}+\left(k_{c}^{\lambda^{\prime \prime \prime}}\right)^{2}+k_{b}^{\lambda^{\prime \prime}} k_{c}^{\lambda^{\prime \prime \prime}}+\left(k_{a}^{\lambda^{\prime}}\right)^{2}\right], \\
& \left(b_{4}^{a b c}\right)^{\lambda^{\prime} \lambda^{\prime \prime} \lambda^{\prime \prime \prime}}=\left[\left(k_{a}^{\lambda^{\prime}}\right)^{2}\left(k_{b}^{\lambda^{\prime \prime}}+k_{c}^{\lambda^{\prime \prime \prime}}\right)\left(\frac{3}{2}-v_{T}\right)+\left\{k_{a}^{\lambda^{\prime}}\left(\left(k_{b}^{\lambda^{\prime \prime}}\right)^{2}+\left(k_{c}^{\lambda^{\prime \prime \prime}}\right)^{2}+k_{b}^{\lambda^{\prime \prime}} k_{c}^{\lambda^{\prime \prime \prime}}\right)+k_{b}^{\lambda^{\prime \prime}} k_{c}^{\lambda^{\prime \prime \prime}}\left(k_{b}^{\lambda^{\prime \prime}}+k_{c}^{\lambda^{\prime \prime \prime}}\right)\right\}\left(\frac{3}{2}-v_{T}\right)^{2}\right], \\
& \left(b_{5}^{a b c}\right)^{\lambda^{\prime} \lambda^{\prime \prime} \lambda^{\prime \prime \prime}}=\left[\left(\frac{3}{2}-v_{T}\right)\left\{\left(k_{a}^{\lambda^{\prime}}\right)^{2}\left(\left(k_{b}^{\lambda^{\prime \prime}}\right)^{2}+\left(k_{c}^{\lambda^{\prime \prime \prime}}\right)^{2}+k_{b}^{\lambda^{\prime \prime}} k_{c}^{\lambda^{\prime \prime \prime}}\right)+\left(k_{b}^{\lambda^{\prime \prime}} k_{c}^{\lambda^{\prime \prime \prime}}\right)^{2}\right\}+\left(\frac{3}{2}-v_{T}\right)^{2} k_{a}^{\lambda^{\prime}} k_{b}^{\lambda^{\prime \prime}} k_{c}^{\lambda^{\prime \prime \prime}}\left(k_{b}^{\lambda^{\prime \prime}}+k_{c}^{\lambda^{\prime \prime \prime}}\right)\right], \\
& \left(b_{6}^{a b c}\right)^{\lambda^{\prime} \lambda^{\prime \prime} \lambda^{\prime \prime \prime}}=\left(\frac{3}{2}-v_{T}\right) k_{a}^{\lambda^{\prime}} k_{b}^{\lambda^{\prime \prime}} k_{c}^{\lambda^{\prime \prime \prime \prime}}\left[k_{b}^{\lambda^{\prime \prime}} k_{c}^{\lambda^{\prime \prime \prime}}+k_{a}^{\lambda^{\prime}}\left(k_{b}^{\lambda^{\prime \prime}}+k_{c}^{\lambda^{\prime \prime \prime}}\right)\right],\left(b_{7}^{a b c}\right)^{\lambda^{\prime} \lambda^{\prime \prime} \lambda^{\prime \prime \prime}}=-\left(k_{a}^{\lambda^{\prime}} k_{b}^{\lambda^{\prime \prime}} k_{c}^{\lambda^{\prime \prime \prime}}\right)^{2}, \\
& O_{1}^{\lambda^{\prime \prime \prime}}=1, O_{2}^{\lambda^{\prime \prime \prime}}=i K^{\lambda^{\prime \prime \prime}}, O_{3}^{\lambda^{\prime \prime \prime}}=-\left(k_{a}^{\lambda^{\prime \prime \prime}} k_{b}+k_{b}^{\lambda^{\prime \prime \prime}} k_{c}+k_{c}^{\lambda^{\prime \prime \prime}} k_{a}\right), O_{4}^{\lambda^{\prime \prime \prime}}=-i k_{a}^{\lambda^{\prime \prime \prime}} k_{b} k_{c},
\end{aligned}
$$

where $k_{1}^{\lambda^{\prime}}=\lambda^{\prime} k_{1}, k_{2}^{\lambda^{\prime \prime}}=\lambda^{\prime \prime} k_{2}$, and $k_{3}^{\lambda^{\prime \prime \prime}}=\lambda^{\prime \prime \prime} k_{3}$.

E. Functions appearing in four-scalar correlator

\section{Contact interaction}

The functional dependence of the momentum dependent functions appearing in the context of contact interaction of the four-scalar correlation can be expressed as 


$$
\begin{aligned}
& G_{1}=\left(\frac{3}{2}-v_{s}\right)^{4}, G_{2}=i \bar{K} G_{1}, G_{3}=G_{1}^{\frac{3}{4}} \sum_{i=1}^{4} k_{i}^{2}+G_{1} \sum_{i>j=1}^{4} k_{i} k_{j}, G_{4}=i G_{1} \sum_{i>j>m=1}^{4} k_{i} k_{j} k_{m}+i G_{1}^{\frac{3}{4}} \sum_{i \neq j=1}^{4} k_{i}^{2} k_{j}, \\
& G_{5}=\sqrt{G_{1}} \sum_{i>j=1}^{4} k_{i}^{2} k_{j}^{2}+G_{1}^{\frac{3}{4}} \sum_{i>j>m=1}^{4} k_{i}^{2} k_{j} k_{m}+G_{1} \prod_{i=1}^{4} k_{i}, G_{6}=i \sqrt{G_{1}} \sum_{i, j, m=1}^{4} k_{i}^{2} k_{j}^{2} k_{m}+i \prod_{i \neq j>m>n=1}^{4} k_{i}^{2} k_{j} k_{m} k_{n}, \\
& G_{7}=G_{1}^{\frac{1}{4}} \prod_{i>j>m=1}^{4} k_{i}^{2} k_{j}^{2} k_{m}^{2}+\sqrt{G_{1}} \prod_{i<j, m<n, i \neq m, j \neq n=1}^{4} k_{i}^{2} k_{j}^{2} k_{m} k_{n}, G_{8}=i G_{1}^{\frac{3}{4}} \prod_{i>j>m \neq n=1}^{4} k_{i}^{2} k_{j}^{2} k_{m}^{2} k_{n}, \\
& G_{9}=\prod_{i=1}^{4} k_{i}^{2}, \overline{\mathcal{Z}}_{1}=1, \overline{\mathcal{Z}}_{2}=i \bar{K}, \overline{\mathcal{Z}}_{3}=\prod_{i>j=1}^{4} k_{i} k_{j}, \overline{\mathcal{Z}}_{4}=\prod_{i>j>m=1}^{4} k_{i} k_{j} k_{m}, \overline{\mathcal{Z}}_{5}=\prod_{i=1}^{4} k_{i}
\end{aligned}
$$

and

$$
\begin{aligned}
& \overline{\mathcal{I}}(i, j ; m, n)=\left[\bar{K}^{4} \sqrt{G_{1}} \Gamma\left(10-6 v_{s}\right)+\bar{K}^{3} \sqrt{G_{1}}\left(k_{i}+k_{j}+k_{m}+k_{n}\right) \Gamma\left(11-6 v_{s}\right)\right. \\
& \quad-\bar{K}^{2}\left\{k_{m}^{2} k_{n}^{2}-i G_{1}^{\frac{1}{4}} k_{m} k_{n}\left(k_{m}+k_{n}\right)-k_{m} k_{n} \sqrt{G_{1}}-k_{i} k_{j} \sqrt{G_{1}}-\left(k_{i}+k_{j}\right)\left(k_{m}+k_{n}\right) \sqrt{G_{1}}\right\} \Gamma\left(12-6 v_{s}\right) \\
& \left.\quad+\bar{K}\left\{k_{i} k_{j}\left(k_{m}+k_{n}\right) \sqrt{G_{1}}-\left(k_{i}+k_{j}\right) k_{m} k_{n} \sqrt{G_{1}}\right\} \Gamma\left(13-6 v_{s}\right)+k_{m} k_{n} \sqrt{G_{1}} \Gamma\left(14-6 v_{s}\right)\right] .
\end{aligned}
$$

\section{Scalar exchange}

The functional dependence of the momentum dependent functions appearing in the context of scalar exchange contribution of the four-scalar correlation can be expressed as

$$
\begin{aligned}
& \Xi_{1}\left(k_{1}, k_{2}, k_{3}, k_{4}, k_{5}, k_{6}\right):=\frac{\left(\frac{3}{2}-v_{s}\right)^{6}}{\left(k_{5} k_{6}\right)^{v_{s}}} \sum_{b=0}^{6} \sum_{p=0}^{6} \mathbf{S}_{b}\left(k_{1}, k_{2}, k_{3}\right) \mathbf{S}_{p}\left(k_{4}, k_{5}, k_{6}\right) \frac{(-1)^{b+p-12 v_{s}}\left(i\left(k_{4}+k_{5}+k_{6}\right)\right)^{b+p-6 v_{s}}}{\left(k_{4}+k_{5}+k_{6}\right)^{4}} \\
& \times \Gamma\left(\frac{3}{2}+b-3 v_{s}\right) \Gamma\left(3+b+p-6 v_{s}\right)_{2} F_{1}^{\mathbf{R E G}}\left[3+b+p-6 v_{s} ; \frac{3}{2}+b-3 v_{s} ; \frac{5}{2}+b-3 v_{s} ;-\frac{\left(k_{1}+k_{2}+k_{3}\right)}{\left(k_{4}+k_{5}+k_{6}\right)}\right],
\end{aligned}
$$

$$
\begin{aligned}
& \Xi_{2}\left(k_{1}, k_{2}, k_{3}, k_{4}, k_{5}, k_{6}\right):=\frac{\left(\frac{3}{2}-v_{s}\right)^{2}}{c_{S}\left(k_{5} k_{6}\right)^{v_{s}}} \sum_{m=0}^{4} \sum_{n=0}^{4} \mathbf{E}_{m}\left(k_{1}, k_{2}, k_{3}\right) \mathbf{E}_{n}\left(k_{4}, k_{5}, k_{6}\right) \frac{(-1)^{2(m+n)-9 v_{s}}\left(i\left(k_{4}+k_{5}+k_{6}\right)\right)^{1-m-n+6 v_{s}}}{\left(7+2 m-6 v_{s}\right)\left(k_{4}+k_{5}+k_{6}\right)^{8}} \\
& \times \Gamma\left(7+m+n-6 v_{s}\right)_{2} F_{1}\left[7+m+n-6 v_{s} ; \frac{7}{2}+m-3 v_{s} ; \frac{9}{2}+b-3 v_{s} ;-\frac{\left(k_{1}+k_{2}+k_{3}\right)}{\left(k_{4}+k_{5}+k_{6}\right)}\right],
\end{aligned}
$$

$$
\begin{aligned}
& \Xi_{3}\left(k_{1}, k_{2}, k_{3}, k_{4}, k_{5}, k_{6}\right):=\frac{c_{S}\left(\frac{3}{2}-v_{s}\right)^{4}}{\left(k_{5} k_{6}\right)^{v_{s}}} \sum_{p=0}^{6} \sum_{q=0}^{4} \mathbf{S}_{p}\left(k_{1}, k_{2}, k_{3}\right) \mathbf{E}_{q}\left(k_{4}, k_{5}, k_{6}\right) \frac{(-1)^{2(p+q)-12 v_{s}+3}\left(i\left(k_{4}+k_{5}+k_{6}\right)\right)^{1-p-q+6 v_{s}}}{\left(k_{4}+k_{5}+k_{6}\right)^{6}} \\
& \times \Gamma\left(\frac{3}{2}+p-3 v_{s}\right) \Gamma\left(5+p+q-6 v_{s}\right)_{2} F_{1}^{\mathbf{R E G}}\left[5+p+q-6 v_{s} ; \frac{3}{2}+p-3 v_{s} ; \frac{5}{2}+p-3 v_{s} ;-\frac{\left(k_{1}+k_{2}+k_{3}\right)}{\left(k_{4}+k_{5}+k_{6}\right)}\right],
\end{aligned}
$$




$$
\begin{aligned}
& \Xi_{4}\left(k_{1}, k_{2}, k_{3}, k_{4}, k_{5}, k_{6}\right):=\frac{c_{S}\left(\frac{3}{2}-v_{s}\right)^{3}}{\left(k_{5} k_{6}\right)^{v_{s}}} \sum_{t=0}^{4} \sum_{r=0}^{6} \mathbf{E}_{t}\left(k_{1}, k_{2}, k_{3}\right) \mathbf{S}_{r}\left(k_{4}, k_{5}, k_{6}\right) \frac{(-1)^{2(t+r)-12 v_{s}+3}\left(i\left(k_{4}+k_{5}+k_{6}\right)\right)^{1-t-r+6 v_{s}}}{\left(k_{4}+k_{5}+k_{6}\right)^{6}} \\
& \times \Gamma\left(\frac{3}{2}+t-3 v_{s}\right) \Gamma\left(5+t+r-6 v_{s}\right)_{2} F_{1}^{\mathbf{R E G}}\left[5+t+r-6 v_{s} ; \frac{3}{2}+t-3 v_{s} ; \frac{5}{2}+t-3 v_{s} ;-\frac{\left(k_{1}+k_{2}+k_{3}\right)}{\left(k_{4}+k_{5}+k_{6}\right)}\right],
\end{aligned}
$$

where we use the regularized hypergeometric function defined as ${ }_{2} F_{1}{ }^{\mathbf{R E G}}[a ; b ; c ; d]=\frac{2 F_{1}[a ; b ; c ; d]}{\Gamma[c]}$. Additionally here we define two new sets of momentum dependent functions given by

$$
\begin{aligned}
& \mathbf{S}_{0}\left(k_{a}, k_{b}, k_{c}\right)=\left(\frac{3}{2}-v_{s}\right)^{3}, \mathbf{S}_{1}\left(k_{a}, k_{b}, k_{c}\right)=-i\left(k_{a}+k_{b}+k_{c}\right)\left(\frac{3}{2}-v_{s}\right)^{3}, \\
& \mathbf{S}_{2}\left(k_{a}, k_{b}, k_{c}\right)=-\left(\frac{3}{2}-v_{s}\right)^{2}\left[\left(k_{a}^{2}+k_{b}^{2}\right)+\left(\frac{3}{2}-v_{s}\right) k_{a} k_{b}\right]-k_{c}\left(k_{a}+k_{b}\right)\left(\frac{3}{2}-v_{s}\right)^{3}-k_{c}^{2}\left(\frac{3}{2}-v_{s}\right)^{2}, \\
& \mathbf{S}_{3}\left(k_{a}, k_{b}, k_{c}\right)=i k_{a} k_{b}\left(k_{a}+k_{b}\right)\left(\frac{3}{2}-v_{s}\right)^{2}+i k_{c}\left(\frac{3}{2}-v_{s}\right)^{2}\left[\left(k_{a}^{2}+k_{b}^{2}\right)+\left(\frac{3}{2}-v_{s}\right) k_{a} k_{b}\right]+i k_{c}^{2}\left(k_{a}+k_{b}\right)\left(\frac{3}{2}-v_{s}\right)^{2}, \\
& \mathbf{S}_{4}\left(k_{a}, k_{b}, k_{c}\right)=k_{a}^{2} k_{b}^{2}\left(\frac{3}{2}-v_{s}\right)^{2}+k_{a} k_{b} k_{c}\left(k_{a}+k_{b}\right)\left(\frac{3}{2}-v_{s}\right)^{2}+\left(\frac{3}{2}-v_{s}\right) k_{c}^{2}\left[\left(k_{a}^{2}+k_{b}^{2}\right)+\left(\frac{3}{2}-v_{s}\right) k_{a} k_{b}\right] \\
& \mathbf{S}_{5}\left(k_{a}, k_{b}, k_{c}\right)=-i k_{a}^{2} k_{b}^{2} k_{c}\left(\frac{3}{2}-v_{s}\right)-i k_{a} k_{b} k_{c}^{2}\left(k_{a}+k_{b}\right)\left(\frac{3}{2}-v_{s}\right), \mathbf{S}_{6}\left(k_{a}, k_{b}, k_{c}\right)=-k_{a}^{2} k_{b}^{2} k_{c}^{2}, \\
& \mathbf{E}_{0}\left(k_{a}, k_{b}, k_{c}\right)=\left(\frac{3}{2}-v_{s}\right), \mathbf{E}_{1}\left(k_{a}, k_{b}, k_{c}\right)=-i\left(k_{a}+k_{b}+k_{c}\right)\left(\frac{3}{2}-v_{s}\right), \mathbf{E}_{2}\left(k_{a}, k_{b}, k_{c}\right)=-k_{a} k_{b}\left(\frac{3}{2}-v_{s}\right) \\
& -k_{a}\left(k_{a}+k_{b}\right)\left(\frac{3}{2}-v_{s}\right)-k_{a}^{2}, \mathbf{E}_{3}\left(k_{a}, k_{b}, k_{c}\right)=i k_{a} k_{b} k_{c}\left(\frac{3}{2}-v_{s}\right)+i\left(k_{a}+k_{b}\right) k_{a}^{2}, \mathbf{E}_{4}\left(k_{a}, k_{b}, k_{c}\right)=k_{a}^{2} k_{b} k_{c},
\end{aligned}
$$

where the superscript indices of the momentum are $a=$ $(1,4), b=(2,5)$, and $c=(3,6)$.

\section{Graviton exchange}

In this context the divergence free contributions of the momentum dependent functions appearing in the context of graviton exchange can be written as

$$
\begin{aligned}
\hat{\vartheta}_{a b c d}+\hat{\vartheta}_{c d a b}= & \frac{k_{a}+k_{b}}{U_{c d}^{2}}\left[\frac{1}{2}\left(U_{c d}+k_{a b}\right)\left(U_{c d}^{2}-2 D_{c d}\right)+k_{a b}^{2}\left(k_{c}+k_{d}\right)\right]+(a, b \leftrightarrow c, d) \\
& +\frac{k_{a} k_{b}}{\bar{K}}\left[\frac{D_{c d}}{U_{c d}}-k_{a b}+\frac{k_{a b}}{U_{a b}}\left(k_{c} k_{d}-k_{a b} \frac{D_{c d}}{U_{c d}}\right)\left(\frac{1}{\bar{K}}+\frac{1}{U_{a b}}\right)\right]+(a, b \leftrightarrow c, d) \\
& -\frac{k_{a b}}{U_{a b} U_{c d} \bar{K}}\left[D_{a b} D_{c d}+2 k_{a b}^{2}\left(\prod_{a} k_{a}\right)\left(\frac{1}{\bar{K}^{2}}+\frac{1}{U_{a b} U_{c d}}+\frac{k_{a b}}{\bar{K} U_{a b} U_{c d}}\right)\right],
\end{aligned}
$$

where we define $U_{a b} \equiv k_{a}+k_{b}+k_{a b}, D_{a b} \equiv\left(k_{a}+k_{b}\right) k_{a b}+$ $k_{a} k_{b}$. 


\section{References}

1. WMAP collaboration, D.N. Spergel et al., Astrophys. J. Suppl. 170, 377 (2007) (for uptodate results on WMAP, see http://lambda.gsfc. nasa.gov/product/map/current)

2. P.A.R. Ade et al., (BICEP2 Collaboration), arXiv:1403.3985 [astro-ph.CO]

3. P.A.R. Ade et al., (Planck Collaboration), arXiv:1303.5082 [astro-ph.CO]

4. P.A.R. Ade et al., (Planck Collaboration), arXiv:1303.5076 [astro-ph.CO]

5. P.A.R. Ade et al., (Planck Collaboration), arXiv:1303.5084 [astro-ph.CO]

6. K. Young Choi, L.M.H. Hall, C. van de Bruck, JCAP 0702, 029 (2007)

7. J.M. Maldacena, G.L. Pimentel, JHEP 1109, 045 (2011)

8. J. Maldacena, JHEP 0305, 013 (2003)

9. J.R. Fergusson, E.P.S. Shellard, Phys. Rev. D 76, 083523 (2007)

10. G.I. Rigopoulos, E.P.S. Shellard, B.J.W. van Tent, Phys. Rev. D 76 , 083512 (2007)

11. G.I. Rigopoulos, E.P.S. Shellard, B.J.W. van Tent, Phys. Rev. D 73, 083522 (2006)

12. G.I. Rigopoulos, E.P.S. Shellard, B.J.W. van Tent, Phys. Rev. D 72, 083507 (2005)

13. X. Chen, R. Easther, E.A. Lim, JCAP 0706, 023 (2007)

14. X. Chen, JCAP 1012, 003 (2010)

15. X. Chen, Adv. Astron. 2010, 638979 (2010)

16. X. Chen, Y. Wang, JCAP 1004, 027 (2010)

17. X. Chen, Y. Wang, Phys. Rev. D 81, 063511 (2010)

18. X. Chen, B. Hu, M.-X. Huang, G. Shiu, Y. Wang, JCAP 0908, 008 (2009)

19. X. Chen, M.-X. Huang, G. Shiu, Phys. Rev. D 74, 121301 (2006)

20. X. Chen, M.-X. Huang, S. Kachru, G. Shiu, JCAP 0701, 002 (2007)

21. X. Chen, Phys. Rev. D 72, 123518 (2005)

22. C.T. Byrnes, M. Sasaki, D. Wands, Phys. Rev. D 74, 123519 (2006)

23. M. Sasaki, J. Valiviita, D. Wands, Phys. Rev. D 74, 103003 (2006)

24. F. Vernizzi, D. Wands, JCAP 0605, 019 (2006)

25. L.E. Allen, S. Gupta, D. Wands, JCAP 0601, 006 (2006)

26. D. Seery, J.E. Lidsey, JCAP 0701, 008 (2007)

27. D. Seery, J.E. Lidsey, Phys. Rev. D 75, 043505 (2007)

28. D. Seery, J.E. Lidsey, M.S. Sloth, JCAP 0701, 027 (2007)

29. D. Seery, J.C. Hidalgo, JCAP 0607, 008 (2006)

30. D. Seery, J.E. Lidsey, JCAP 0606, 001 (2006)

31. D. Seery, J.E. Lidsey, JCAP 0509, 011 (2005)

32. D. Seery, J.E. Lidsey, JCAP 0506, 003 (2005)

33. T. Battefeld, R. Easther, JCAP 0703, 020 (2007)

34. P. Creminelli, L. Senatore, M. Zaldarriaga, JCAP 0703, 019 (2007)
35. L. Senatore, M. Zaldarriaga, JCAP 1101, 003 (2011)

36. J. Yoo, N. Hamaus, U. Seljak, M. Zaldarriaga, Phys. Rev. D 86, 063514 (2012)

37. P. Creminelli, G. D’Amico, M. Musso, J. Norea, E. Trincherini, JCAP 1102, 006 (2011)

38. P. Creminelli, A. Nicolis, L. Senatore, M. Tegmark, M. Zaldarriaga, JCAP 0605, 004 (2006)

39. K.A. Malik, D.H. Lyth, JCAP 0609, 008 (2006)

40. D.H. Lyth, JCAP 0511, 006 (2005)

41. L. Boubekeur, D.H. Lyth, Phys. Rev. D 73, 021301 (2006)

42. D.H. Lyth, Y. Rodriguez, Phys. Rev. Lett. 95, 121302 (2005)

43. D.H. Lyth, Y. Rodriguez, Phys. Rev. D 71, 123508 (2005)

44. N. Bartolo, S. Matarrese, A. Riotto, JCAP 0508, 010 (2005)

45. A. De Felice, S. Tsujikawa, Phys. Rev. D 84, 083504 (2011)

46. A. De Felice, S. Tsujikawa, JCAP 1104, 029 (2011)

47. S. Mizuno, K. Koyama, Phys. Rev. D 82, 103518 (2010)

48. T. Kidani, K. Koyama, S. Mizuno, arXiv: 1207.4410

49. S. Renaux-Petel, S. Mizuno, K. Koyama, JCAP 11, 042 (2011)

50. S. Mizuno, K. Koyama, Phys. Rev. D 82, 103518 (2010)

51. T. Kobayashi, M. Yamaguchi, J. Yokoyama, Phys. Rev. D 83, 103524 (2011)

52. T. Kobayashi, M. Yamaguchi, J. Yokoyama, Prog. Theor. Phys. 126, $511(2011)$

53. S. Renaux-Petel, arXiv: 1105.6366

54. J. Martin, L. Sriramkumar, arXiv: 1109.5838

55. C. de Rahm, A.J. Tolley, JCAP 1005, 015 (2010)

56. G.L. Goon, K. Hinterbichler, M. Trodden, Phys. Rev. D 83, 085015 (2011)

57. G.L. Goon, K. Hinterbichler, M. Trodden, JCAP 07, 017 (2011)

58. M. Cederwall, A. von Gussich, A. Mikovi, B.E.W. Nilsson, A. Westerberg. Phys. Lett. B 390, 148 (1997)

59. E.J. Copeland, S. Mizuno, M. Shaeri, Phys. Rev. D 81, 123501 (2010)

60. S. Choudhury, S. Pal, Nucl. Phys. B 874, 85 (2013)

61. K.M. Smith, M. LoVerde, M. Zaldarriaga, Phys. Rev. Lett. 107, 191301 (2011)

62. N.S. Sugiyama, E. Komatsu, T. Futamase, Phys. Rev. Lett. 106, $251301(2011)$

63. D. Baumann, arXiv:0907.5424

64. Y. Rodriguez, J.P. Beltran Almeida, C.A. Valenzuela-Toledo, arXiv: 1301.5843

65. M.H. Namjoo, H. Firouzjahi, M. Sasaki, arXiv:1210.3692

66. Y. Rodriguez, J.P.B. Almeida, C.A. Valenzuela-Toledo, JCAP 1304, 039 (2013)

67. C.T. Byrnes, E.J. Copeland, A.M. Green, Phys. Rev. D 86, 043512 (2012)

68. P.A. Klimai, E.V. Bugaev, arXiv:1210.3262 